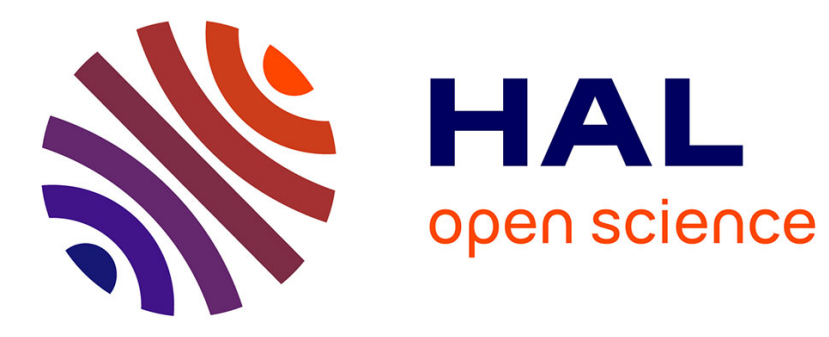

\title{
Smash Products of Calabi-Yau Algebras by Hopf Algebras
}

\author{
Patrick Le Meur
}

\section{To cite this version:}

Patrick Le Meur. Smash Products of Calabi-Yau Algebras by Hopf Algebras. Journal of Noncommutative Geometry, 2019, 13 (3), pp.887-961. 10.4171/JNCG/341 . hal-01314228v2

\section{HAL Id: hal-01314228 \\ https://hal.science/hal-01314228v2}

Submitted on 14 Nov 2019

HAL is a multi-disciplinary open access archive for the deposit and dissemination of scientific research documents, whether they are published or not. The documents may come from teaching and research institutions in France or abroad, or from public or private research centers.
L'archive ouverte pluridisciplinaire HAL, est destinée au dépôt et à la diffusion de documents scientifiques de niveau recherche, publiés ou non, émanant des établissements d'enseignement et de recherche français ou étrangers, des laboratoires publics ou privés. 


\title{
SMASH PRODUCTS OF CALABI-YAU ALGEBRAS BY HOPF ALGEBRAS
}

\author{
PATRICK LE MEUR
}

\begin{abstract}
Let $H$ be a Hopf algebra and $A$ be an $H$-module algebra. This article investigates when the smash product $A \sharp H$ is (skew) Calabi-Yau, has Van den Bergh duality or is Artin-Schelter regular or Gorenstein. In particular, if $A$ and $H$ are skew Calabi-Yau, then so is $A \sharp H$ and its Nakayama automorphism is expressed using the ones of $A$ and $H$. This is based on a description of the inverse dualising complex of $A \sharp H$ when $A$ is a homologically smooth $\mathrm{dg}$ algebra and $H$ is homologically smooth and with invertible antipode. This description is also used to explain the compatibility of standard constructions of Calabi-Yau dg algebras with taking smash products.
\end{abstract}

\section{INTRODUCTION}

The Calabi-Yau algebras were defined in 13 and are now widely investigated. They appear in deformations of unimodular Poisson structures (see 4, 11 and [33]). In noncommutative geometry, many relevant Artin-Schelter regular algebras are Calabi-Yau, like the Sklyannin algebras. The Calabi-Yau algebras also appear as noncommutative resolutions of singularities, for instance, as Jacobian algebras arising from brane tilings $(28)$ or as skew group algebras of polynomial algebras (see [2] and [9]). Finally, there are general constructions of candidates for being Calabi-Yau dg algebras, such as the Ginzburg dg algebras of [13] or, more generally, the (deformed) Calabi-Yau completions of [21, which are used in the construction of cluster categories and their generalisations (see [1] and [22]).

In these frameworks, many algebras of interest take the shape of a smash product. While the works of Yekutieli ([38]) and of Brown and Zhang ([6]) have shown that many interesting Hopf algebras have a duality which is (weaker than and) close to being Calabi-Yau, Reyes, Rogalski and Zhang initiated the study of the algebras having this weaker duality (and called skew Calabi-Yau algebras) by proving that, in the connected graded setting, being skew Calabi-Yau is, on one hand, equivalent to being Artin-Schelter regular and, on the other hand, relatively stable under taking smash products with finite-dimensional Hopf algebras.

This motivates the work done in this article, which is, for a Hopf algebra $H$ and an $H$-module differential graded algebra $A$, to determine if $A \sharp H$ is Calabi-Yau (or, in case $A$ is an algebra, if $A \sharp H$ has Van den Bergh duality or is skew Calabi-Yau).

Let $\mathbb{k}$ be a field. A differential graded $(\mathrm{dg})(\mathbb{k}$-)algebra $A$ is called $n$-Calabi-Yau if it is homologically smooth (that is, $A \in \operatorname{per}\left(A^{e}\right)$ ) and $\operatorname{RHom}_{A^{e}}\left(A, A^{e}\right)[n] \simeq A$ in

Date: November 8, 2019.

2010 Mathematics Subject Classification. Primary 16E65, 16S40; Secondary 16E40, 16E45, $18 \mathrm{G} 10$

Key words and phrases. Hopf algebra, smash product, Calabi-Yau algebra, skew Calabi-Yau algebra, Van den Bergh duality, Nakayama automorphism, homological determinant, weak homological determinant.

First published in: Le Meur Patrick, Smash Products of Calabi-Yau Algebras by Hopf Algebras. J. Noncommut. Geom. 13 (2019), 887-961. DOI 10.4171/JNCG/341. (C) European Mathematical Society. 
the derived category $\mathcal{D}\left(A^{e}\right)$ of $A^{e}=A \underset{\mathbb{k}}{\otimes} A^{\text {op }}$. Often, a cofibrant replacement of $\mathrm{RHom}_{A^{e}}\left(A, A^{e}\right)$ is referred to as an inverse dualising complex of $A$.

When $A$ is a $\mathbb{k}$-algebra, being Calabi-Yau means that $A$ admits a finite resolution by finitely generated projective left $A^{e}$-modules (or, $A$-bimodules), and $\operatorname{Ext}_{A^{e}}^{i}\left(A, A^{e}\right)$ is isomorphic to $A$ as an $A$-bimodule if $i=n$ and is zero otherwise. Recall the following weaker forms of duality.

- A has Van den Bergh duality in dimension $n$ if it is homologically smooth and the $A$-bimodule $\operatorname{Ext}_{A^{e}}^{i}\left(A, A^{e}\right)$ is invertible if $i=n$ and is zero otherwise.

- $A$ is skew Calabi-Yau in dimension $n$ when it has Van den Bergh duality in dimension $n$ and, moreover, $\operatorname{Ext}_{A^{e}}^{n}\left(A, A^{e}\right)$ is isomorphic to $A^{\mu_{A}}={ }^{1} A^{\mu_{A}}$ as an $A$-bimodule, for some automorphism $\mu_{A} \in \operatorname{Aut}_{\mathrm{k}-\mathrm{alg}}(A)$.

The naming in the former case refers to the sufficient conditions for the duality theorem of Van den Bergh on the Hochschild (co)homology of $A$ to hold true (see [34, Theorem 1]). In the latter case, $\mu_{A}$ is called a Nakayama automorphism. It is uniquely determined up to the composition with an inner automorphism. As usual, given $\mathbb{k}$-algebra homomorphisms $\tau, \sigma: A \rightarrow A$, the piece of notation ${ }^{\tau} A^{\sigma}$ stands for the $\mathbb{k}$-vector space $A$ with $A$-bimodule structure given by $a \cdot x \cdot b=\tau(a) x \sigma(b)$.

This article hence describes an inverse dualising complex of $A \sharp H$ when $A$ is a homologically smooth dg algebra acted on by a homologically smooth Hopf algebra $H$ with invertible antipode. On one hand, when $H$ is involutive, this description is applied to express the deformed Calabi-Yau completions of $A \sharp H$ in terms of smash products with $H$ of the deformed Calabi-Yau completions of $A$. On the other hand, when $A$ is an algebra, this description is applied to give necessary and/or sufficient conditions for $A \sharp H$ to have Van den Bergh duality or to be skew Calabi-Yau (with an explicit Nakayama automorphism). As a consequence, the Nakayama automorphisms of Artin-Schelter regular algebras have trivial homological determinants as conjectured in [30, Conjecture 6.4]. Also, explicit Nakayama automorphisms are computed for the smash products arising from actions of finite-dimensional Lie algebras on polynomial algebras and from actions of $\mathcal{U}_{q}\left(\mathfrak{s l}_{2}\right)$ on the quantum plane.

In this article, $H$ denotes a Hopf algebra with antipode $S$ and $A$ denotes an $H$-module dg algebra. " $A$ is an algebra" means that $A$ is concentrated in degree 0 as a dg algebra. The smash product $A \sharp H$ is denoted by $\Lambda$. When $A$ is augmented (or connected $(\mathbb{N}$-) graded), it is also assumed that the augmentation ideal is an $H$ submodule of $A$ (or that the action of $H$ on $A$ preserves the grading, respectively).

\section{Main Results AND StRUCTURE OF THE ARTiCle}

Assuming that $S$ is invertible is convenient and many Hopf algebras which are relevant to the dualities considered here have this property (see [6]). This is the case of noetherian and Calabi-Yau Hopf algebras (see [15, Theorem 2.3] whose proof can be adapted to noetherian Hopf algebras with Van den Bergh duality). This is actually the case for a broader class of Hopf algebras.

Proposition 1 (3.4.2). Any Hopf algebra with Van den Bergh duality has an invertible antipode.

When $S$ is invertible and $H$ is noetherian, it is proved in [6] that, if $H$ has Van den Bergh duality or is Artin-Schelter regular, then it is skew Calabi-Yau with $S^{-2} \circ \Xi_{\int_{\ell}}^{r}$ as a Nakayama automorphism. Here, $\int_{r}$ is the right homological integral of $H$ and $\Xi_{\int_{r}}^{r}$ is the corresponding right winding automorphism of $H$. Combining this result and Proposition 1 yields the following characterisation.

Theorem 1 3.5.1, 3.5.2, 3.5.3). Let $H$ be a Hopf algebra with antipode $S$. The following conditions are equivalent 
(i) H has Van den Bergh duality,

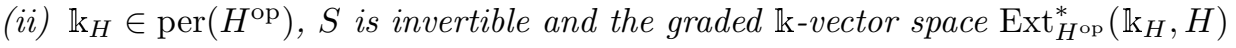
is finite-dimensional and concentrated in one degree,

(iii) $H$ is skew Calabi-Yau.

Under any of these conditions, $S^{-2} \circ \Xi_{\int_{r} \circ S}^{r}$ is a Nakayama automorphism of $H$. In particular, $H$ is Calabi-Yau if and only if $\mathbb{k}_{H} \in \operatorname{per}\left(H^{\mathrm{op}}\right), S^{2}$ is an inner automorphism of $H$ and $H$ has the right Artin-Schelter property with trivial right homological integral.

Note that it is proved in [15. Theorem 2.3] that, when $H$ is noetherian, $H$ is Calabi-Yau if and only if $S^{2}$ is an inner automorphism and $H$ is Artin-Schelter regular with trivial left homological integral.

This article is based on the description of $\operatorname{RHom}_{\Lambda^{e}}\left(\Lambda, \Lambda^{e}\right)$. When $S$ is invertible, there exists a $\operatorname{dg} A$-bimodule $D_{A}$ which is $H_{S^{2}}$-equivariant in the sense of [30] (see Section (4) and such that $D_{A} \simeq \operatorname{RHom}_{A^{e}}\left(A, A^{e}\right)$ in $\mathcal{D}\left(A^{e}\right)$. A suitable extension of $D_{A}$ is then isomorphic to $\mathrm{RHom}_{\Lambda^{e}}\left(\Lambda, \Lambda^{e}\right)$ in the following sense. See 5.5.1 for a general statement. See also [12, 14, 15, 16, 26, 30, 36, 37, for previous results describing $\operatorname{RHom}_{\Lambda^{e}}\left(\Lambda, \Lambda^{e}\right)$ when $A$ is a connected graded algebra and $H$ is finitedimensional, semisimple or cocommutative.

Proposition 2 (5.5.2). Let $H$ be a Hopf algebra with Van den Bergh duality in dimension d. Let $A$ be an $H$-module $d g$ algebra. Assume that $A$ is homologically smooth. Then, $\Lambda$ is homologically smooth and $\operatorname{RHom}_{\Lambda^{e}}\left(\Lambda, \Lambda^{e}\right) \simeq D_{A} \sharp^{\sigma} H[-d]$ where $\sigma=\left(S^{-2} \circ \Xi_{\int_{\ell}}^{r}\right)^{-1}=S^{2} \circ \Xi_{\int_{\ell} \circ S}^{r}$.

Here, $D_{A} \sharp^{\sigma} H$ is the $\operatorname{dg} \Lambda$-bimodule associated to the $H_{S^{2}}$-equivariant $\operatorname{dg} A$ bimodule $D_{A}$ and defined in [30] (see 4.2 for a reminder).

The description of $\mathrm{RHom}_{\Lambda^{e}}\left(\Lambda, \Lambda^{e}\right)$ can be used to describe the deformed CalabiYau completions of $\Lambda$. Recall that $\operatorname{HH}_{n-2}(A) \simeq H^{0} \operatorname{Hom}_{A^{e}}\left(D_{A}[n-1], A[1]\right)$ when $D_{A}$ is cofibrant over $A^{e}$, which is possible to assume. The following result was proved in [25] when $H$ is the (semisimple) group algebra of a finite group.

Theorem 2 6.1, 6.3). Let $H$ be an involutive Hopf algebra which is moreover Calabi-Yau in dimension d. Let $A$ be a homologically smooth $H$-module dg algebra. Let $n \in \mathbb{Z}$.

(1) The $n$-Calabi-Yau completion $\Pi_{n}(A)$ is an $H$-module $d g$ algebra and the $d g$ algebras $\Pi_{n}(A) \sharp H$ and $\Pi_{n+d}(A \sharp H)$ are isomorphic.

(2) Given a deformed Calabi-Yau completion $\Pi_{n}(A, \alpha)$ such that $\alpha \in \mathrm{HH}_{n-2}(A)$ arises from an $H$-linear cocycle $D_{A}[n-1] \rightarrow A[1]$, then $\Pi_{n}(A, \alpha)$ is an $H$ module $d g$ algebra and there is an associated $\bar{\alpha} \in \mathrm{HH}_{n+d-2}(\Lambda)$ such that $\Pi_{n}(A, \alpha) \sharp H \simeq \Pi_{n+d}(A \sharp H, \bar{\alpha})$.

The reader is referred to 6.1 and 6.2 .3 for generalisations to the case where $H$ is merely a Hopf algebra with Van den Bergh duality.

When $A$ is an algebra, Proposition 2 can also be used to characterise when $\Lambda$ has Van den Bergh duality.

Theorem 3 (7.1). Let $A$ be an $H$-module algebra where $H$ is a Hopf algebra. Assume that the antipode $S$ is invertible and that both $A$ and $H$ are homologically smooth. Then, the following assertions are equivalent

(i) $A$ and $H$ have Van den Bergh duality,

(ii) $\Lambda$ has Van den Bergh duality.

When these conditions are satisfied and $n, d$ are the corresponding homological dimensions of $A$ and $H$, respectively, then $\Lambda$ has dimension $n+d$ and

$$
\operatorname{Ext}_{\Lambda^{e}}^{n+d}\left(\Lambda, \Lambda^{e}\right) \simeq \operatorname{Ext}_{A^{e}}^{n}\left(A, A^{e}\right) \sharp{ }^{\left(S^{-2} \circ \Xi_{S_{\ell}}^{r}\right)^{-1}} H .
$$


This characterisation specialises to skew Calabi-Yau algebras. A Nakayama automorphism of $A \sharp H$ was proved to exist and was described in [30, Theorem 0.2] when $A$ is noetherian, connected graded, and skew Calabi-Yau and $H$ is finitedimensional. This uses the homological determinant (hdet: $H \rightarrow \mathbb{k}$ ) of the action of $H$ on $A$ (in the sense of [18, 23]). In general, hdet is not defined and [30, Question 7.2] asked for an extension of its definition. As a partial answer, the concept of weak homological determinant whdet: $H \rightarrow A$ is introduced in 7.2 when $A$ is skew CalabiYau. It is determined by the choice of a free generator of $\operatorname{Ext}_{A^{e}}^{n}\left(A, A^{e}\right)$ in $\bmod (A)$ and it defines an algebra homomorphism $\theta_{\text {whdet }}: H \rightarrow \Lambda$ by $h \mapsto \operatorname{whdet}\left(S^{2}\left(h_{1}\right)\right) h_{2}$ (this replaces $\Xi_{\text {hdet }}^{\ell}$ when hdet is not defined). The following result extends 30 , Theorem 0.2] which was mentioned previously and answers 30, Question 4.3]. See 7.2.2.4 and Table 4 for examples where whdet takes values outside $\mathbb{k}$.

Theorem 4 7.3.1 and 7.3.2. Let $H$ be a Hopf algebra with invertible antipode. Let $A$ be an $H$-module algebra. Assume that $A$ and $H$ are homologically smooth.

(1) If $A$ and $H$ are skew Calabi-Yau, then so is $\Lambda$.

(2) If $\Lambda$ is skew Calabi-Yau, then so is $H$ and the action of $H$ on $A$ has a weak homological determinant. If, moreover, a homological determinant exists, then $A$ is skew Calabi-Yau.

In the setting of (1), then $\Lambda$ admits as a Nakayama automorphism

$$
\mu_{\Lambda}=\mu_{A} \sharp\left(\theta_{\text {whdet }} \circ \mu_{H}\right)
$$

where $\mu_{A}$ is a Nakayama automorphism of $A$, whdet: $H \rightarrow A$ is an associated weak homological determinant, and $\mu_{H}=S^{-2} \circ \Xi_{\int_{\ell}}^{r}$.

Here, for given mappings $\alpha: A \rightarrow \Lambda$ and $\beta: H \rightarrow \Lambda$, the piece of notation $\alpha \sharp \beta$ denotes the mapping $\Lambda \rightarrow \Lambda, a h \mapsto \alpha(a) \beta(h)$.

Should whdet take its values in $\mathbb{k}$ then $\mu_{\Lambda}=\mu_{A} \sharp\left(\Xi_{\text {whdet }}^{\ell} \circ \mu_{H}\right)$. In particular, when $A$ is connected graded and skew Calabi-Yau, then a generator of $\operatorname{Ext}_{A^{e}}^{n}\left(A, A^{e}\right)$ may be chosen such that whdet $=$ hdet. This yields characterisations of when $A \sharp H$ is Calabi-Yau assuming that $H$ is so. See [15, Theorem 3.4] for a characterisation of when $\mathcal{U}(\mathfrak{g}) \sharp \mathbb{k} k$ is Calabi-Yau, for finite-dimensional Lie algebras $\mathfrak{g}$ and finite groups $G$ (in zero characteristic), note that $\mathcal{U}(\mathfrak{g})$ need not be graded and that $\mathbb{k} G$ is then semisimple and Calabi-Yau in dimension 0.

Theorem 5 (7.4.2). Let $H$ be a Calabi-Yau Hopf algebra. Let $A$ be a connected graded $H$-module algebra. Let $h_{0} \in H^{\times}$be such that $S^{-2}$ is the inner automorphism of $h_{0}$ (see Theorem 1). Then, $\Lambda=A \sharp H$ is Calabi-Yau if and only if the following conditions hold

(a) A is skew Calabi-Yau,

(b) hdet $=\epsilon$,

(c) $\left(\exists k_{A} \in Z\left(H^{\times}\right)\right)(\forall a \in A) \quad \mu_{A}(a)=\left(h_{0} k_{A}\right) \rightarrow a \underset{\text { in } \Lambda}{=}\left(h_{0} k_{A}\right) a\left(h_{0} k_{A}\right)^{-1}$.

When $A$ is, moreover, Calabi-Yau, Theorem 5 simplifies as follows.

Corollary 1 (7.4.3). Let $H$ be a Calabi-Yau Hopf algebra. Let $A$ be a connected graded $H$-module algebra which is moreover Calabi-Yau. The following assertions are equivalent

(i) $A \sharp H$ is Calabi-Yau,

(ii) hdet $=\epsilon$.

This characterisation was proved previously in the following situations

- in 37. assuming that $A$ is $p$-Koszul Calabi-Yau and that $H=\mathbb{k} G$ for any finite subgroup $G$ of $\operatorname{Aut}_{\mathbb{k}-\operatorname{alg}}(A)$ such that car $(\mathbb{k})$ does not divide $\operatorname{Card}(G)$,

- in [26] assuming that $A$ is $p$-Koszul and Calabi-Yau and that $S^{2}=\operatorname{Id}_{H}$, 
- in [16, Corollary 3.4] assuming that $A$ is $p$-Koszul and Calabi-Yau and $H=(\mathbb{k} G)^{*}$ for any finite group $G$.

It was conjectured in [30, Conjecture 6.4] that the Nakayama automorphisms of all connected graded Artin-Schelter Gorenstein algebras have trivial homological determinant and this was proved for noetherian and connected graded Artin-Schelter regular algebras in [31, Corollary 5.4]. Combining Theorem 5 and the main result of [14], it is possible to prove that the noetherian hypothesis is unnecessary.

Corollary 2 (7.4.4). Let $A$ be a connected graded Artin-Schelter regular algebra (equivalently, a connected graded skew Calabi-Yau algebra, see [30, Lemma 1.2]). Let $\mu_{A}$ be its (graded) Nakayama automorphism. Let $H=\mathbb{k} \mathbb{Z}$ and consider the action of $H$ on $A$ induced by $\mu_{A}$. Then, $\operatorname{hdet}\left(\mu_{A}\right)=1$.

The above mentioned conjecture was proved previously in the following cases,

- in [30, Theorem 0.4], for noetherian connected graded Koszul Artin-Schelter regular algebras;

- in [31, Corollary 5.4], for noetherian and connected graded Artin-Schelter Gorenstein algebras of one of the following shapes

- graded twists of algebras which are finite over their affine centres

- quotients of noetherian Artin-Schelter regular algebras;

- in [27, Theorem 1.6], for $m$-Koszul Artin-Schelter regular algebras;

- in 8, Theorem 3.11], for certain 4 dimensional connected graded ArtinSchelter regular algebras which are normal extensions of 3 dimensional ones.

The last main result of this text gives sufficient conditions for $\Lambda$ to be ArtinSchelter Gorenstein/regular when $A$ is an augmented $H$-module algebra. Note that the hypotheses below entail that the antipode of $H$ is invertible.

Theorem 6 9.3.3 9.3.5. Let $H$ be a Hopf algebra. Let $A$ be an augmented $H$ module algebra which is moreover noetherian. Assume that $A$ is Artin-Schelter Gorenstein in dimension $n$.

(1) If gl.dim. $A<\infty$ and $H$ has Van den Bergh duality in dimension d, then $\Lambda$ is Artin-Schelter regular in dimension $n+d$.

(2) If $\operatorname{dim}_{\mathrm{k}} H<\infty$, then $\Lambda$ is Artin-Schelter Gorenstein in dimension n.

(3) If $H$ has Van den Bergh duality in dimension d, then $A \sharp H$ is Artin-Schelter Gorenstein in dimension $n+d$.

Part (2) was proved in [30, Theorem 4.1] when $A$ is connected graded ArtinSchelter Gorenstein. Besides [16, Proposition 3.8] proved that, when $H$ is finitedimensional and semi-simple and $A$ is an $H$-module dg algebra concentrated in nonnegative degrees and with zero component equal to $\mathbb{k}$, then $A$ is Artin-Schelter Gorenstein if and only if so is $A \sharp H$.

This article is organised as follows. Section 2 recalls useful definitions, it sets conventions and it proves useful folklore results. Section 3 proves Proposition 1 and

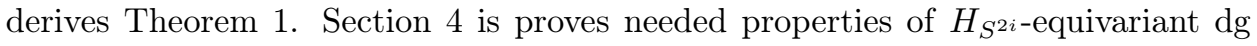
$A$-bimodules $(i \in \mathbb{Z})$. Section 5 is devoted to the description of $\operatorname{RHom}_{\Lambda^{e}}\left(\Lambda, \Lambda^{e}\right)$ and the proof of Proposition 2. Section 6 applies this description to the compatibility of deformed Calabi-Yau completions with taking smash products, it proves Theorem2. Section 7 uses this description to prove Theorem 3 and Theorem 4 As a corollary it proves Theorem 5 and Corollary 1 . The results of this section are applied in Section 8 to the computation of a Nakayama automorphism of $A \sharp H$ when $A=$ $\mathbb{C}_{q}[x, y]$ and $H=\mathcal{U}_{q}\left(\mathfrak{s l}_{2}\right)\left(q \in \mathbb{C}^{\times}\right.$not being a root of unity). Finally, Section 9 concentrates on the case where $A$ is an augmented $\mathbb{k}$-algebra. It proves Theorem 6 ,

For the ease of reading, an index of notation is provided at the end of the article. 


\section{BASIC DEFINITIONS AND CONVENTIONS}

2.1. Conventions on notation. For simplicity, $\otimes_{\mathbb{k}}$ is denoted by $\otimes$.

The counit of $H$ is denoted by $\epsilon$. The Sweedler notation $h_{1} \otimes h_{2}$ is used for the comultiplication of $h \in H$, omitting the summation symbol. The action of $h \in H$ on an element $x$ of a left (or right) $H$-module is written as $h \rightarrow x$ (or, $x<h$, respectively).

The category of left $\operatorname{dg} A$-modules is denoted by $\mathcal{C}(A)$. And $\mathcal{C}\left(A^{\text {op }}\right)$ is identified with the category of right $\operatorname{dg} A$-modules. The derived category of $A$ is denoted by $\mathcal{D}(A)$ and defined as the localisation of $\mathcal{C}(A)$ at the class of all quasi-isomorphisms. The perfect derived category of $A$ is denoted by $\operatorname{per}(A)$ and defined as the smallest triangulated subcategory of $\mathcal{D}(A)$ containing $A$ and stable under taking direct summands. When $A$ is an algebra, the category of left $A$-modules is denoted by $\bmod (A)$. And $\bmod \left(A^{\text {op }}\right)$ is identified with the category of right $A$-modules.

For all $X \in \mathcal{C}(A)$, the suspension of $X$ is denoted by $X[1]$. For all $X, Y \in \mathcal{C}(A)$, then $\operatorname{Hom}_{A}(X, Y)$ denotes the following complex of vector spaces

- for $n \in \mathbb{Z}$, its component of degree $n$ is the vector space of (homogeneous of degree zero) morphisms of graded vector spaces $f: X \rightarrow Y[n]$ such that $f(a x)=(-1)^{n \cdot \operatorname{deg}(a)} a f(x)$ for all homogeneous $x \in X$ and $a \in A$,

- the differential is given by $f \mapsto d_{Y} \circ f-(-1)^{\operatorname{deg}(f)} f \circ d_{X}$.

Hence, the morphism space $\mathcal{C}(A)(X, Y)$ equals $Z^{0} \operatorname{Hom}_{A}(X, Y)$.

No difference is made between $\mathrm{dg} A$-bimodules and left $\operatorname{dg} A^{e}$-modules. For such a $\operatorname{dg} A$-bimodule $M$, the identity $a_{1} m a_{2}=(-1)^{\operatorname{deg}(m) \operatorname{deg}\left(a_{2}\right)}\left(a_{1} \otimes a_{2}\right) \cdot m$ holds when $m \in M$ and $a_{2} \in A$ are homogeneous. In particular, given $M, N \in \mathcal{C}\left(A^{e}\right)$, $n \in \mathbb{Z}$ and $f \in \operatorname{Hom}_{\mathbb{k}}(M, N)^{n}$, then $f \in \operatorname{Hom}_{A^{e}}(M, N)$ if and only if $f(a x b)=$ $(-1)^{n \cdot \operatorname{deg}(a)} a f(x) b$, for every $a \in A$ homogeneous, $x \in X$ and $b \in B$.

Here is a reminder of the features of $\mathcal{C}(A)$ (see [20, for details). A dg module $P \in \mathcal{C}(A)$ is cofibrant if, for every surjective quasi-isomorphism $X \rightarrow Y$ in $\mathcal{C}(A)$, then any morphism $P \rightarrow Y$ in $\mathcal{C}(A)$ lifts to $X$. There exists a model structure on $\mathcal{C}(A)$ whose class of weak equivalences consists of all the quasi-isomorphisms, and whose class of cofibrant objects consists of all the cofibrant left $\operatorname{dg} A$-modules. In particular,

- for every $X \in \mathcal{C}(A)$ there exists a quasi-isomorphism $P \rightarrow X$ in $\mathcal{C}(A)$ where $P$ is cofibrant (such a $P$ is called a cofibrant replacement of $X$ ),

- for every $P, X \in \mathcal{C}(A)$ such that $P$ is cofibrant, the canonical mapping $H^{0} \operatorname{Hom}_{A}(P, X) \rightarrow \mathcal{D}(A)(P, X)$ is bijective,

- every cofibrant $P \in \mathcal{C}(A)$ is homotopically projective, that is, for every quasi-isomorphism $X \rightarrow Y$ in $\mathcal{C}(A)$, then $\operatorname{Hom}_{A}(P, X) \rightarrow \operatorname{Hom}_{A}(P, Y)$ is a quasi-isomorphism.

Fibrant dg modules are defined dually and feature dual properties. In particular, they are homotopically injective.

The two following basic facts are used without further reference in this article.

Lemma. Let $A, B$ be $d g$ algebras.

(1) Let $A \rightarrow B$ be a morphism of dg algebras. If $B \simeq A \otimes V$ in $\mathcal{C}(A)$ for some complex of vector spaces $V$, then the restriction-of-scalars functor $\mathcal{C}(B) \rightarrow \mathcal{C}(A)$ preserves cofibrant objects.

(2) Let $A \rightarrow B$ be a morphism of dg algebras. Then, the extension-of-scalars functor $\mathcal{C}(A) \rightarrow \mathcal{C}(B)$ preserves cofibrant objects.

(3) The restriction-of-scalars functor $\mathcal{C}\left(A \otimes B^{\text {op }}\right) \rightarrow \mathcal{C}(A)$ preserves fibrant objects. 
For the sake of simplicity, the verifications of module structures omit the obvious quantifiers and use implicitly lower case letters for elements in a space named with the corresponding upper case letter $(x \in X, y \in Y$, etc.). Also $h, k, \ell$ always denote elements of $H$, whereas $a, b$ always denote homogeneous elements of $A$. As for the equalities presented as "identities", and which may involve parameters $(a, h, d$, etc.), it is implicit that they hold true for all possible values of the parameters $(\forall a \in A$, $\forall h \in H, \forall d \in D$, etc.).

\subsection{The smash product $\Lambda=A \sharp H$.}

2.2.1. Recall that a structure of $H$-module $\mathrm{dg}$ algebra on $A$ is a morphism of complexes of vector spaces $H \otimes A \rightarrow A, h \otimes a \mapsto h \rightarrow a$ (with $H$ in degree 0) such that the following identities hold true in $A$

$$
\begin{aligned}
& 1 \rightarrow a=a, \quad h \rightarrow(a b)=\left(h_{1} \rightarrow a\right)\left(h_{2} \rightarrow b\right), \\
& h \rightarrow 1=\epsilon(h), \quad(h k) \rightarrow a=h \rightarrow(k \rightarrow a) .
\end{aligned}
$$

The dg algebra $A \sharp H$ has $A \otimes H$ as underlying complex of vector spaces. A tensor $a \otimes h$ is denoted by $a h$. The (associative) product of $A \sharp H$ is given by

$$
(a h) \times(b k)=(a \otimes h) \times(b \otimes k)=a\left(h_{1} \rightarrow b\right) \otimes h_{2} k=a\left(h_{1} \rightarrow b\right) h_{2} k .
$$

2.2.2. Assume that $S$ is invertible. The following identities hold in $\Lambda^{e}$

$$
\begin{aligned}
& (h \otimes k) \times(a \otimes b)=\left(\left(h_{1} \rightarrow a\right) \otimes\left(S^{-1}\left(k_{1}\right) \rightarrow b\right)\right) \times\left(h_{2} \otimes k_{2}\right) \\
& (a \otimes b) \times(h \otimes k)=\left(h_{2} \otimes k_{2}\right) \times\left(\left(S^{-1}\left(h_{1}\right) \rightarrow a\right) \otimes\left(k_{1} \rightarrow b\right)\right) .
\end{aligned}
$$

The algebra $H^{\mathrm{op}}$ is a Hopf algebra with coproduct given by $h \mapsto h_{1} \otimes h_{2}$ and antipode $S^{-1}$. Also $H^{e}$ is a Hopf algebra with coproduct given by $(h \otimes k)_{1} \otimes(h \otimes$ $k)_{2}=\left(h_{1} \otimes k_{1}\right) \otimes\left(h_{2} \otimes k_{2}\right)$ and antipode $S \otimes S^{-1}$.

There is a structure of $\operatorname{dg} H^{e}$-module algebra on $A^{e}$ such that

$$
(h \otimes k) \rightarrow(a \otimes b)=(h \rightarrow a) \otimes\left(S^{-1}(k) \rightarrow b\right) .
$$

The resulting smash product dg algebra $A^{e} \sharp H^{e}$ is isomorphic to $\Lambda^{e}$ via the mapping $A^{e} \otimes H^{e} \rightarrow \Lambda^{e}$ defined by $a \otimes b \otimes h \otimes k \mapsto(a \otimes b) \times(h \otimes k)$. In other words, the following identity holds true in $\Lambda^{e}$

$$
(h \otimes k) \times(a \otimes b)=(h \otimes k)_{1} \rightarrow(a \otimes b) \times(h \otimes k)_{2} .
$$

The natural structure of left $\operatorname{dg} A^{e}$-module of $A^{e}$ extends to a structure of left $\operatorname{dg} \Lambda^{e}$-module such that $(a h \otimes b k) \rightarrow(x \otimes y)=(-1)^{\operatorname{deg}(b)(\operatorname{deg}(x)+\operatorname{deg}(y))}(a(h \rightarrow$ $x)) \otimes\left(S^{-1}(k) \rightarrow(y b)\right)$.

This structure and the structure of right $\mathrm{dg} A^{e}$-module of $A^{e}$ do not form a structure of $\Lambda^{e}-A^{e}$-bimodule. Instead, those two structures are compatible in the following sense (where $m \in A^{e}$ )

$$
\begin{aligned}
& (h \otimes k) \rightarrow(m<(a \otimes b))=\left((h \otimes k)_{1} \rightarrow m\right)<\left((h \otimes k)_{2} \rightarrow(a \otimes b)\right) \\
& \left(a^{\prime} \otimes b^{\prime}\right) \rightarrow(m<(a \otimes b))=\left(\left(a^{\prime} \otimes b^{\prime}\right) \rightarrow m\right)<(a \otimes b) .
\end{aligned}
$$

(which is part of the identities defining the $H^{e}$-module dg algebra structure on $A^{e}$ ).

\subsection{Duality conditions on $\mathbf{d g}$ algebras. Let $n$ be a natural integer.}

2.3.1. The definitions of the dualities considered in this article are recalled in the introduction. Note that, given a $\operatorname{dg} A^{e}$-bimodule $X$, then $\operatorname{Hom}_{A^{e}}(A, X)$ is a $\mathrm{dg}$ $A$-bimodule. Whence the functor $\operatorname{Hom}_{A^{e}}(A,-): \mathcal{C}\left(A^{e} \otimes\left(A^{e}\right)^{\mathrm{o} p}\right) \rightarrow \mathcal{C}\left(A^{e}\right)$. This is how $\operatorname{RHom}_{A^{e}}\left(A, A^{e}\right)$ is considered as an object of $\mathcal{D}\left(A^{e}\right)$. When $A$ is a $\mathbb{k}$-algebra, $\operatorname{Ext}_{A^{e}}^{i}\left(A, A^{e}\right)$ inherits of a structure of $A$-bimodule for every $i$. Recall that, if $A$ is a $\mathbb{k}$-algebra, then $A$ is Calabi-Yau if and only if it is skew Calabi-Yau and any Nakayama automorphism for $A$ is inner (equivalently, the identity map of $A$ is a Nakayama automorphism). 
2.3.2. When $A$ is a skew Calabi-Yau algebra in dimension $n$, every free generator $e$ of $\operatorname{Ext}_{A^{e}}^{n}\left(A, A^{e}\right)$ in $\bmod (A)$ determines a unique Nakayama automorphism $\mu \in$ $\operatorname{Aut}_{\mathrm{k}-\mathrm{alg}}(A)$ such that the identity $e a=\mu(a) e$ holds in $\operatorname{Ext}_{A^{e}}^{n}\left(A, A^{e}\right)$.

Lemma. Let $A$ be a $\mathbb{k}$-algebra. Let $D \in \bmod \left(A^{e}\right)$ be such that there exists $\nu \in$ $\operatorname{Aut}_{\mathrm{k}-\mathrm{alg}}(A)$ verifying $D \simeq A^{\nu}$ in $\bmod \left(A^{e}\right)$. Let $d \in D$ be a free generator of $D$ in $\bmod (A)$ and denote by $\mu: A \rightarrow A$ the algebra homomorphism such that the identity $d a=\mu(a) d$ holds in $D$. Then, $\mu \in \operatorname{Aut}_{\mathbb{k}-\operatorname{alg}}(A)$ and $D \simeq A^{\mu}$ in $\bmod \left(A^{e}\right)$.

Proof. The mapping $A^{\mu} \rightarrow D, a \mapsto a d$ is an isomorphism in $\bmod \left(A^{e}\right)$. It therefore suffices to prove that $\mu$ is bijective. There exists a free generator $d^{\prime} \in D$ in $\bmod (A)$ such that the identity $d^{\prime} a=\nu(a) d^{\prime}$ holds in $D$. Let $\alpha \in A^{\times}$be such that $d^{\prime}=\alpha d$. Then, $\mu(a) d=d a=\alpha^{-1} d^{\prime} a=\alpha^{-1} \nu(a) d^{\prime}=\alpha^{-1} \nu(a) \alpha d$. Therefore, $\mu \circ \nu^{-1} \in$ $\operatorname{Aut}_{\mathrm{k}-\mathrm{alg}}(A)$, and hence $\mu \in \operatorname{Aut}_{\mathrm{k}-\mathrm{alg}}(A)$.

2.3.3. When $A$ is moreover connected $(\mathbb{N}$-)graded, it is required that there exists $\ell \in \mathbb{Z}$ and a homogeneous $\mu_{A} \in \operatorname{Aut}_{\mathrm{k}-\operatorname{alg}}(A)$ such that $\operatorname{Ext}_{A^{e}}^{n}\left(A, A^{e}\right) \simeq A^{\mu_{A}}(\ell)$ as graded $A$-bimodules for $A$ to be considered as skew Calabi-Yau in the graded sense. Here, $-(\ell)$ denotes the degree shift of graded modules. The following lemma is used later on. Its proof is elementary and omitted.

Lemma. Let $A$ be a connected graded $\mathbb{k}$-algebra. Assume that $A$ is skew Calabi-Yau in the ungraded sense, then so is it in the graded sense.

2.3.4. Assume that $A$ is an augmented algebra. Then, $A$ is said to satisfy the left Artin-Schelter condition in dimension $n$ if

$$
\operatorname{dim}_{\mathbb{k}} \operatorname{Ext}_{A}^{i}\left({ }_{A} \mathbb{k}, A\right)= \begin{cases}1 & \text { for } i=n \\ 0 & \text { otherwise. }\end{cases}
$$

The right Artin-Schelter condition is defined analogously using right $A$-modules instead of left $A$-modules. When $A$ satisfies both the left and right Artin-Schelter conditions, then $A$ is said to satisfy the Artin-Schelter condition.

The algebra $A$ is called Artin-Schelter Gorenstein when it satisfies the ArtinSchelter condition and the injective dimensions of ${ }_{A} A$ and $A_{A}$ are finite and equal. It is called Artin-Schelter regular when, moreover, gl.dim. $(A)<\infty$.

\section{Homological dualities for Hopf algebras}

The objective of this section is to prove Proposition 1 the antipode of $H$ is invertible when $H$ has Van den Bergh duality. This is done in Section 3.4. For this purpose, a brief reminder on winding automorphisms is given in Section 3.1, next, a general description of an inverse dualising complex of $H$ is given in Section 3.2 . and a useful characterisation of homologically smooth Hopf algebras is proved in Section 3.3 Some consequences regarding Calabi-Yau duality and Nakayama automorphisms are discussed in Section 3.5. In particular, Theorem 1 is proved there.

3.1. Winding automorphisms (see [6, Section 4.5]). If $H$ satisfies the right Artin-Schelter condition in dimension $d$, then $\operatorname{Ext}_{H^{\text {op }}}^{d}\left(\mathbb{k}_{H}, H\right)$ is called the right homological integral of $H$ and denoted by $\int_{r}$. The algebra homomorphism $\pi: H \rightarrow$ $\mathbb{k}$ such that the left $H$-module structure of $\operatorname{Ext}_{H^{\text {op }}}^{d}\left(\mathbb{k}_{H}, H\right)$ is given by $h \rightarrow \alpha=$ $\pi(h) \alpha$ is also denoted by $\int_{r}$. It is called trivial if $\int_{r}=\epsilon$ as maps $H \rightarrow \mathbb{k}$. The left homological integral $\int_{\ell}$ is defined analogously using $\operatorname{Ext}_{H}^{d}\left({ }_{H} \mathbb{k}, H\right)$.

Let $\pi: H \rightarrow \mathbb{k}$ be any algebra homomorphism. The following mappings

$$
\begin{aligned}
& \Xi_{\pi}^{\ell}: H \rightarrow H \quad \Xi_{\pi}^{r}: H \rightarrow H \\
& h \mapsto \pi\left(h_{1}\right) h_{2} \quad h \mapsto h_{1} \pi\left(h_{2}\right)
\end{aligned}
$$


are algebra automorphism with respective inverses $\Xi_{\pi \circ S}^{\ell}$ and $\Xi_{\pi \circ S}^{r}$. Since $\pi \circ S^{2}=\pi$ (see [30, (E1.2.2)]), then both $\Xi_{\pi}^{\ell}$ and $\Xi_{\pi}^{r}$ commute with $S^{2}$. The automorphisms $\Xi_{\pi}^{\ell}$ and $\Xi_{\pi}^{r}$ are the left and right winding automorphisms of $\pi$.

Example. Let $\mathfrak{g}$ be a $d$-dimensional Lie algebra $(d \in \mathbb{N})$. Assume that $H$ is the universal enveloping algebra $\mathcal{U}(\mathfrak{g})$. Following [38, Corollary 2.2], there is an isomorphism of right $H$-modules $\operatorname{Ext}_{H}^{d}\left({ }_{H} \mathbb{k}, H\right) \simeq \Lambda^{d} \mathfrak{g}^{*}$. In particular

$$
(\forall X \in \mathfrak{g}) \quad \int_{\ell}(X)=\operatorname{Tr}\left(\operatorname{ad}_{X}\right) .
$$

3.2. The inverse dualising complex of a Hopf algebra. The result below describes $\mathrm{RHom}_{H^{e}}\left(H, H^{e}\right)$ in terms of $\mathrm{RHom}_{H^{\mathrm{op}}}\left(\mathbb{k}_{H}, H\right)$. Given any left $H$-module $N$, denote by $N \uparrow H^{e}$ the $H$-bimodule with underlying vector space $H \otimes N$ and with action by $H^{e}$ given by $h(\ell \otimes n) k=S^{2}\left(h_{1}\right) \ell k \otimes\left(h_{2} \rightarrow n\right)$. Note that $H \uparrow H^{e} \simeq H^{e}$ in $\bmod \left(H^{e}\right)$; More precisely, the mapping $H \uparrow H^{e} \rightarrow H^{e}$ defined by $\ell \otimes n \mapsto n_{2} \otimes S\left(n_{1}\right) \ell$ is an isomorphism of $H$-bimodules with inverse given by $h \otimes k \mapsto S^{2}\left(h_{1}\right) k \otimes h_{2}$. The assignment $N \mapsto N \uparrow H^{e}$ defines an exact functor $\bmod (H) \rightarrow \bmod \left(H^{e}\right)$ preserving projectives. It is isomorphic to the extension-of-scalars functor along the algebra homomorphism $H \rightarrow H^{e}$ given by $h \mapsto h_{2} \otimes S\left(h_{1}\right)$. The resulting total derived functor $\mathcal{D}(H) \rightarrow \mathcal{D}\left(H^{e}\right)$ is also denoted by $N \mapsto N \uparrow H^{e}$.

Proposition. Let $H$ be a Hopf algebra. Assume that $\mathbb{k}_{H}$ has a resolution in $\bmod \left(H^{\mathrm{op}}\right)$ by finitely generated projectives. Then,

$$
\operatorname{RHom}_{H^{e}}\left(H, H^{e}\right) \simeq \mathrm{RHom}_{H^{\mathrm{op}}}\left(\mathbb{k}_{H}, H\right) \uparrow H^{e} \text { in } \mathcal{D}\left(\left(H^{e}\right)^{\mathrm{op}}\right) \text {. }
$$

Proof. Let $P \rightarrow \mathbb{k}_{H}$ be a resolution in $\bmod \left(H^{\mathrm{op}}\right)$ by finitely generated projectives. Hence, $\operatorname{RHom}_{H^{\mathrm{op}}}\left(\mathbb{k}_{H}, H\right) \simeq \operatorname{Hom}_{H^{\mathrm{op}}}(P, H)$ in $\mathcal{D}(H)$. The stated isomorphism is proved in three steps. First, by proving that a projective resolution $\bar{P}$ of $H$ in $\bmod \left(H^{e}\right)$ can be deduced from $P$. Next, by proving that $\operatorname{RHom}_{H^{e}}\left(H, H^{e}\right) \simeq$ $\operatorname{Hom}_{H \text { op }}(P, H \otimes H)$ (with an adequate structure of right $H$-module on $\left.H \otimes H\right)$. Finally, by proving that $\operatorname{Hom}_{H^{\mathrm{op}}}(P, H \otimes H) \simeq \operatorname{Hom}_{H^{\mathrm{op}}}(P, H) \uparrow H^{e}$.

Step 1 - Given $X \in \bmod \left(H^{\mathrm{op}}\right)$, denote by $\bar{X}$ the $H$-bimodule equal to $H \otimes X$ as a vector space and with actions of $H$ given by $h(\ell \otimes x) k=h \ell k_{1} \otimes x<k_{2}$. Then,

- $\overline{H_{H}} \simeq H^{e}$ in $\bmod \left(H^{e}\right)$; More precisely, the mapping $\overline{H_{H}} \rightarrow H^{e}$ defined by $\ell \otimes x \mapsto \ell S\left(x_{1}\right) \otimes x_{2}$ is an isomorphism of $H$-bimodules with inverse given by $h \otimes k \mapsto h k_{1} \otimes k_{2}$,

- $\overline{\mathbb{k}_{H}} \simeq H$ in $\bmod \left(H^{e}\right)$.

Thus, $\bar{P} \rightarrow \overline{\mathrm{k}_{H}} \simeq H$ is a projective resolution in $\bmod \left(H^{e}\right)$. Hence, $\operatorname{RHom}_{H^{e}}\left(H, H^{e}\right)$ is isomorphic to $\operatorname{Hom}_{H^{e}}\left(\bar{P}, H^{e}\right)$ in $\mathcal{D}\left(H^{e}\right)$. Here, $\operatorname{Hom}_{H^{e}}\left(\bar{P}, H^{e}\right)$ is a complex of $H$-bimodules in the following sense: $(h f k)(-)=f(-) \times(k \otimes h) \in H^{e}$ if $f \in$ $\operatorname{Hom}_{H^{e}}\left(\bar{P}, H^{e}\right), h, k \in H$.

Step 2 - The following mapping

$$
\begin{aligned}
\operatorname{Hom}_{H^{e}}\left(\bar{P}, H^{e}\right) & \rightarrow \operatorname{Hom}_{H^{\mathrm{op}}}(P, H \otimes H) \\
f & \mapsto f(1 \otimes-)
\end{aligned}
$$

is well-defined provided that $H \otimes H$ is considered as a right $H$-module for the action such that $(h \otimes k)<\ell=S\left(\ell_{1}\right) h \otimes k \ell_{2}$. Indeed, this follows from the identity $(1 \otimes p<h)=S\left(h_{1}\right)(1 \otimes p) h_{2}$ in $\bar{P}$. This mapping is moreover bijective with inverse the mapping $\operatorname{Hom}_{H^{\mathrm{op}}}(P, H \otimes H) \rightarrow \operatorname{Hom}_{H^{e}}\left(\bar{P}, H^{e}\right)$ which assigns to any $g$ the morphism of $H$-bimodules $\bar{P} \rightarrow H^{e}$ defined by $\ell \otimes p \mapsto \ell g(p)$. Finally, it is an isomorphism of $H$-bimodules if $\operatorname{Hom}_{H^{\text {op }}}(P, H \otimes H)$ is endowed with the action of $H^{e}$ such that $(h f k)(-)=f(-) \times(k \otimes h) \in H^{e}$ (hence $H \otimes H$ is a right $H \otimes H^{e}$-module). Thus, $\operatorname{RHom}_{H^{e}}\left(H, H^{e}\right) \simeq \operatorname{Hom}_{H^{\text {op }}}(P, H \otimes H)$ in $\mathcal{D}\left(H^{e}\right)$. 
Step 3 - In order to get the announced description of $\operatorname{RHom}_{H^{e}}\left(H, H^{e}\right)$, it is necessary to transform $\operatorname{Hom}_{H^{\mathrm{op}}}(P, H \otimes H)$. Note that $\operatorname{Hom}_{H^{\mathrm{op}}}(P, H)$ is a complex of left $H$-modules in a natural way. This defines $\operatorname{Hom}_{H^{\mathrm{op}}}(P, H) \uparrow H^{e}$. The following mapping is well-defined

$$
\begin{aligned}
\operatorname{Hom}_{H^{\mathrm{op}}}(P, H) \uparrow H^{e} & \rightarrow \operatorname{Hom}_{H^{\mathrm{op}}}(P, H \otimes H) \\
\ell \otimes \theta & \mapsto\left(p \mapsto S\left(\theta(p)_{1}\right) \ell \otimes \theta(p)_{2}\right)
\end{aligned}
$$

It is moreover a morphism of complexes of $H$-bimodules. When $P$ is replaced by $H$, then it identifies with the mapping $H \otimes H \rightarrow H \otimes H$ defined by $\ell \otimes \theta \mapsto S\left(\theta_{1}\right) \ell \otimes \theta_{2}$; This is an isomorphism with inverse given by $h \otimes k \mapsto S^{2}\left(k_{1}\right) h \otimes k_{2}$. Since $P$ consists of finitely generated projective $H$-modules, it follows that the complexes of $H$-bimodules $\operatorname{Hom}_{H^{\mathrm{op}}}(P, H) \uparrow H^{e}$ and $\operatorname{Hom}_{H^{\mathrm{op}}}(P, H \otimes H)$ are isomorphic. Thus, $\operatorname{RHom}_{H^{e}}\left(H, H^{e}\right) \simeq \operatorname{Hom}_{H^{\text {op }}}(P, H) \uparrow H^{e}$.

Keep the setting of the previous result. Taking cohomology shows that, for every $n \in \mathbb{N}$, there is an isomorphism of $H$-bimodules

$$
\operatorname{Ext}_{H^{e}}^{n}\left(H, H^{e}\right) \simeq H \otimes \operatorname{Ext}_{H^{\text {op }}}^{n}\left(\mathbb{k}_{H}, H\right)
$$

where the right hand-side term is endowed with the structure of $H$-bimodule such that $h(\ell \otimes e) k=S^{2}\left(h_{1}\right) \ell k \otimes h_{2} \rightarrow e$. See [15, Corollary 2.2] (and [6, Section 4.5]) for a previous similar description when $H$ is Artin-Schelter Gorenstein (and with invertible antipode, respectively).

3.3. Homological smoothness of Hopf algebras. The following result seems to be well-known at least when $H$ is noetherian. See [29, Lemma 2.4] for a proof using that $S$ is invertible. In the present situation, where $S$ need not be invertible, it follows from the properties of the functor ? $\bmod \left(H^{\mathrm{op}}\right) \rightarrow \bmod \left(H^{e}\right)$ considered in the proof in 3.2 and from the corresponding ones of $\mathbb{k}_{H_{H}}: \bmod \left(H^{e}\right) \rightarrow \bmod \left(H^{\mathrm{op}}\right)$.

Lemma. The Hopf algebra $H$ is homologically smooth if and only if $\mathbb{k}_{H} \in \operatorname{per}\left(H^{\mathrm{op}}\right)$. If it is so, then $\operatorname{pd}_{H^{e}}(H)=\operatorname{pd}_{H^{\text {op }}}\left(\mathbb{k}_{H}\right)$.

\subsection{Invertibility of the antipode.}

3.4.1. A sufficient condition for the right Artin-Schelter property. The following lemma is a key-step in the proof of Proposition 2, it is very similar to the lemma in [6. Section 1.2] except that the condition that $S$ is invertible is dropped here. The proof here is adapted from the proof there.

Lemma. Let $H$ be a Hopf algebra. Assume the following:

(a) $\operatorname{id}\left({ }_{H} H\right)<\infty$,

(b) $\mathbb{k}_{H}$ has a resolution in $\bmod \left(H^{\mathrm{op}}\right)$ by finitely generated projective modules,

(c) there exists $d \in \mathbb{N}$ such that $\operatorname{Ext}_{H \text { op }}^{i}\left(\mathbb{k}_{H}, H\right)$ is finite dimensional if $i=d$ and zero otherwise.

Then, $\operatorname{dim}_{\mathbb{k}} \operatorname{Ext}_{H}^{d}\left({ }_{H} \mathbb{k}, H\right)=\operatorname{dim}_{\mathbb{k}} \operatorname{Ext}_{H^{\mathrm{op}}}^{d}\left(\mathbb{k}_{H}, H\right)=1$.

Proof. According to [6, Section 3.2], conditions (a) and (b) grant the existence of Ischebeck's spectral sequence $([17,1.8])$

$$
E_{2}^{p, q}:=\operatorname{Ext}_{H}^{p}\left(\operatorname{Ext}_{H^{\mathrm{op}}}^{-q}\left(\mathbb{k}_{H}, H\right), H\right) \Rightarrow \operatorname{Tor}_{-p-q}^{H}\left(\mathbb{k}_{H}, H\right)= \begin{cases}\mathbb{k} & \text { if } p+q=0 \\ 0 & \text { otherwise }\end{cases}
$$

Because of condition (c), the spectral sequence degenerates at $E_{2}$. In particular,

$$
\operatorname{dim}_{\mathbb{k}} \operatorname{Ext}_{H}^{d}\left(\operatorname{Ext}_{H^{\mathrm{op}}}^{d}\left(\mathbb{k}_{H}, H\right), H\right)=1 .
$$

Following the ideas in the proof of [5, Lemma 1.11], denote by $V$ the finite dimensional left $H$-module $\operatorname{Ext}_{H^{\circ \mathrm{p}}}^{d}\left(\mathbb{k}_{H}, H\right)$. Endow $\operatorname{Hom}_{\mathbb{k}}(V, H)$ with its usual structure of left $H$-module. The canonical bijection $\operatorname{Hom}_{\mathbb{k}}(V, H) \stackrel{\sim}{\longrightarrow} \operatorname{Hom}_{\mathbb{k}}\left(\mathbb{k}, \operatorname{Hom}_{\mathbb{k}}(V, H)\right)$ 
restricts to an isomorphism $\operatorname{Hom}_{H}(V, H) \stackrel{\sim}{\rightarrow} \operatorname{Hom}_{H}\left(H \mathbb{k}, \operatorname{Hom}_{k}(V, H)\right)$. Deriving this isomorphism yields that $\operatorname{Ext}_{H}^{d}(V, H) \simeq \operatorname{Ext}_{H}^{d}\left({ }_{H} \mathbb{k}, \operatorname{Hom}_{\mathrm{k}}(V, H)\right)$ (see [5, Proposition 1.3]). Besides, consider $H \otimes V^{*}$ as a left $H$-module in the usual way also. The canonical mapping $H \otimes V^{*} \rightarrow \operatorname{Hom}_{\mathrm{k}}(V, H)$ is $H$-linear. And it is bijective since $\operatorname{dim}_{\mathrm{k}} V<\infty$. Therefore, $\operatorname{Ext}_{H}^{d}\left({ }_{H} \mathbb{k}, \operatorname{Hom}_{\mathrm{k}}(V, H)\right) \simeq \operatorname{Ext}_{H}^{d}\left({ }_{H} \mathbb{k}, H \otimes V^{*}\right)$. Now, the left $H$-module $H \otimes V^{*}$ (with action given by $h \rightarrow(\ell \otimes \varphi)=h_{1} \rightarrow \ell \otimes h_{2} \rightarrow \varphi$ ) is isomorphic to the free of rank $\operatorname{dim}_{\mathbb{k}} V$ left $H$-module $H \otimes V$ with action given by $h \rightarrow(\ell \otimes \varphi)=h \ell \otimes \varphi$. Indeed, the mapping from the former to the latter defined by $\ell \otimes \varphi \mapsto \ell_{1} \otimes S\left(\ell_{2}\right) \rightarrow \varphi$ is an isomorphism in $\bmod (H)$ with inverse given by $\ell \otimes \varphi \mapsto \ell_{1} \otimes \ell_{2} \rightarrow \varphi$. Thus,

$$
\operatorname{Ext}_{H}^{d}(V, H) \simeq \operatorname{Ext}_{H}^{d}\left({ }_{H} \mathbb{k}, H\right)^{\operatorname{dim} V} .
$$

Combining (3.4.1.1) and (3.4.1.2) yields the announced equalities.

3.4.2. The following result entails Proposition 2, Part (2) is proved in 6, Section 4.4] when $H$ is Artin-Schelter Gorenstein and noetherian and $S$ is invertible.

Proposition. If a Hopf algebra $H$ has Van den Bergh duality in dimension d, then

(1) $H$ is right Artin-Schelter regular in dimension d, and

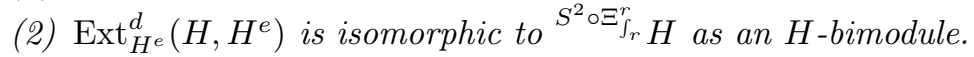

As a consequence, the antipode $S$ is invertible.

Proof. Note that $H$ has finite global dimension equal to $d$ because $H \in \operatorname{per}\left(H^{e}\right)$ and that $d=\operatorname{pd}_{H^{e}}(H)$. There exists a projective resolution $P \rightarrow \mathbb{k}_{H}$ in $\bmod \left(H^{\mathrm{op}}\right)$ having length $d$ and consisting of finitely generated projective right $H$-modules (see 3.3). Consider the resulting complex of $H$-bimodules $\bar{P}$ as introduced in the proof in 3.2. In particular, $\bar{P}$ is a projective resolution with length $d$ of $H$ in $\bmod \left(H^{e}\right)$. Note that $\operatorname{Hom}_{H^{\mathrm{op}}}(P, H)$ is a complex of projective left $H$-modules concentrated in degrees $0, \ldots, d$. Similarly, $\operatorname{Hom}_{H^{\mathrm{op}}}(P, H) \uparrow H^{e}$ is a complex of projective right $H^{e}$-modules whose cohomology is $\operatorname{Ext}_{H^{e}}^{*}\left(H, H^{e}\right)$ (see 3.2).

(1) Denote by $U$ the right $H^{e}$-bimodule $\operatorname{Ext}_{H^{e}}^{d}\left(H, H^{e}\right)$. Because of the assumption on the length of $P$, and since $H$ has Van den Bergh duality, there is a quasiisomorphism in $\mathcal{C}\left(H^{e}\right)$

$$
\operatorname{Hom}_{H^{\mathrm{op}}}(P, H) \uparrow H^{e} \rightarrow U[-d] .
$$

This is a homotopy equivalence in $\bmod \left(H^{\mathrm{op}}\right)$ because both sides are bounded complexes of projective right $H$-modules. Applying $-\underset{H}{\otimes} \mathbb{k}$ therefore yields a quasiisomorphism in $\mathcal{C}(H)$

$$
\left(\operatorname{Hom}_{H^{\mathrm{op}}}(P, H) \uparrow H^{e}\right) \underset{H}{\otimes} H \mathbb{k} \rightarrow U[-d] \underset{H}{\otimes} H \mathbb{k} .
$$

A direct computation shows that $\left(\operatorname{Hom}_{H^{\mathrm{op}}}(P, H) \uparrow H^{e}\right) \underset{H}{\otimes} H_{\mathbb{k}} \simeq \operatorname{Hom}_{H^{\mathrm{op}}}(P, H)$ in $\mathcal{C}(H)$. Consequently,

$$
\operatorname{Ext}_{H^{\text {op }}}^{i}\left(\mathbb{k}_{H}, H\right) \simeq \begin{cases}U \underset{H}{\otimes} \mathbb{k}^{\mathbb{k}} & \text { if } i=d \\ 0 & \text { otherwise. }\end{cases}
$$

Now, as any invertible $H$-bimodule, $U$ is finitely generated in $\bmod \left(H^{\mathrm{op}}\right)$. Applying $-\underset{H}{\otimes} \mathbb{k}_{\mathrm{k}}$ to a free of finite rank cover of $U$ in $\bmod \left(H^{\mathrm{op}}\right)$ yields that $U \underset{H}{\otimes} \mathbb{H}_{\mathrm{k}}$ is finite dimensional. Therefore, 3.4.1 applies here. Thus, $H$ is right Artin Schelter regular in dimension $d$.

(2) As explained earlier, $U \simeq H^{d}\left(\operatorname{Hom}_{H^{\text {op }}}(P, H) \uparrow H^{e}\right)$. The functor $\bmod (H) \rightarrow$ $\bmod \left(H^{e}\right)$ defined by $N \mapsto N \uparrow H^{e}$ is exact. Therefore, $H^{d}\left(\operatorname{Hom}_{H^{\circ \mathrm{p}}}(P, H) \uparrow H^{e}\right) \simeq$ 
$\operatorname{Ext}_{H^{\text {op }}}^{d}\left(\mathbb{k}_{H}, H\right) \uparrow H^{e}$ in $\bmod \left(H^{e}\right)$. Moreover, the construction in 3.2 and the definition of $\int_{r}: H \rightarrow \mathbb{k}_{k}$ yield that $\operatorname{Ext}_{H^{\circ \mathrm{op}}}^{d}\left(\mathbb{k}_{H}, H\right) \uparrow H^{e} \simeq{ }^{2} \circ \Xi_{\int_{r}}^{r} H$ in $\bmod \left(H^{e}\right)$. Thus, $U \simeq S^{2} \circ \Xi_{\int_{r}}^{r} H$. This proves (2).

Since $U$ is invertible as an $H$-bimodule and $U \simeq S^{2} \circ \Xi_{f_{r}}^{r} H$ in $\bmod \left(H^{e}\right)$, then $S^{2} \circ \Xi_{\int_{r}}^{r}$ is an automorphism of $H$. And hence so is $S^{2}$. Thus, $S$ is invertible.

3.5. On Van den Bergh duality of Hopf algebras. For later purposes, here are some consequences of 3.4 .2 many of which were proved in [6], 15, and 30] assuming that $S$ is invertible and/or that $H$ is noetherian.

3.5.1. Summary on Van den Bergh duality. The following characterisation is obtained from 3.4 and from the main results in [6]. When $S$ is assumed to be invertible and $H$ to be noetherian, the same result is already proved in [30, Lemma 1.3].

Theorem. Let $H$ be a Hopf algebra. The following conditions are equivalent

(i) $H$ has Van den Bergh duality,

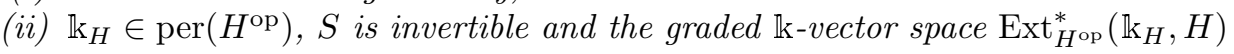
is finite-dimensional and concentrated in one degree,

(iii) $H$ is skew Calabi-Yau.

Under any of these conditions, the homological dimensions involved in $(i),($ ii) and (iii) coincide and $S^{-2} \circ \Xi_{\int_{r} \circ S}^{r}$ is a Nakayama automorphism of $H$.

Proof. The implication $(i) \Rightarrow(i i)$ is proved in 3.4 .2 and 3.3 .

The implication $($ ii $) \Rightarrow($ iii $)$ is proved in [6] when $H$ is noetherian and may be adapted to the present situation. Here is a proof for the convenience of the reader. Assume $(i i)$. Then, $H$ is homologically smooth (see 3.3 ). Let $d \in \mathbb{N}$ be such that $\operatorname{dim}_{\mathbb{k}} \operatorname{Ext}_{H^{\mathrm{op}}}^{i}\left(\mathbb{k}_{H}, H\right)$ is finite if $i=d$ and 0 otherwise. Therefore, $\operatorname{dim}_{\mathbb{k}} \operatorname{Ext}_{H^{\mathrm{op}}}^{d}\left(\mathbb{k}_{H}, H\right)=1$ (see 3.4.1). It follows from 3.2 that $\operatorname{Ext}_{H^{e}}^{i}\left(H, H^{e}\right)$ is isomorphic to $S^{2} \circ \Xi_{\int_{r}}^{r} H \simeq H^{S^{-2}{ }^{\circ} \Xi_{f_{r}}^{r} \circ S}$ in $\bmod \left(H^{e}\right)$ when $i=d$ and is 0 otherwise. This proves that $(i i) \Rightarrow(i i i)$.

The implication $($ iii $) \Rightarrow(i)$ follows from the definition.

3.5.2. Nakayama automorphisms. Using 3.5.1 yields the following relationship between the right homological integral and the Nakayama automorphisms of $H$. Part (1) was proved in [6, Section 0.3] assuming that $S$ is invertible and $H$ is noetherian.

Proposition. If a Hopf algebra $H$ has Van den Bergh duality in dimension d, then the antipode is invertible and

(1) $\left(S^{2} \circ \Xi_{\int_{r}}^{r}\right)^{-1}=S^{-2} \circ \Xi_{\int_{r} \circ S}^{r}$ is a Nakayama automorphism of $H$.

(2) If $\mu \in \operatorname{Aut}_{\mathrm{k}-\mathrm{alg}}(H)$ is any Nakayama automorphism of $H$ then $\epsilon \circ \mu^{-1}$ is the right homological integral of $H$,

Proof. (1) follows from 3.5.1. (2) follows from (1) and from the fact that Nakayama automorphisms differ from one another by an inner automorphism.

3.5.3. Calabi-Yau duality. Combining 3.5.1 and 3.5.2 yields the following.

Corollary. Let $H$ be a Hopf algebra. Then $H$ is Calabi-Yau in dimension $d$ if and only if the following conditions hold

(a) $\mathbb{k}_{H} \in \operatorname{per}\left(H^{\mathrm{op}}\right)$,

(b) $S^{2}$ is an inner automorphism of $H$,

(c) $H$ is satisfies the right Artin-Schelter condition and its right homological integral is trivial. 


\section{Equivariant modules}

This section develops properties and constructions based on $\mathrm{dg} A$-bimodules

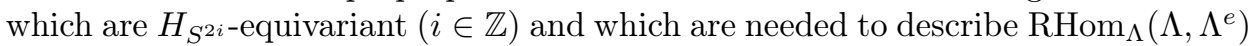
(Section 5), to describe the deformed Calabi-Yau completions of $\Lambda$ (Section 6), and to describe the Nakayama automorphisms of $\Lambda$ (Section 7). Section 4.1 introduces a dg algebra $\Delta_{i}$ such that $\mathcal{C}\left(\Delta_{i}\right)$ consists of those equivariant dg bimodules. Section 4.2 gives details on certain extension-of-scalars functors $D \mapsto D \sharp^{\sigma} H$ from $\mathcal{C}\left(\Delta_{i}\right)$ to $\mathcal{C}\left(\Lambda^{e}\right)$. Section 4.3 explains how $H$ acts on morphism spaces between $H_{S^{2 i}}$-equivariant dg $A$-bimodules. Sections 4.4 and 4.5 detail the behaviour of $H_{S^{2 i}}$-equivariant $\mathrm{dg} A$-bimodules under $\operatorname{Hom}_{A}(-, A)$ and under tensor products over $A$, respectively. Finally, Section 4.6 proves that such a bimodule $D$ is invertible over $A$ if and only if $D \sharp^{\sigma} H$ is invertible over $\Lambda$.

$\Delta_{0}$ was introduced in [19, Definition 3.1] and applied to smash products whether to investigate Calabi-Yau duality (see [26, 30]) or Hochschild cohomology (see [29]).

\subsection{Dg algebras which $\mathrm{dg}$ modules are equivariant $A$-bimodules.}

Definition. Let $i \in \mathbb{Z}$ and assume that $S$ is invertible when $i<0$. Define $\Delta_{i}$ to be the dg algebra whose underlying complex of vector spaces is $A^{e} \otimes H$ and whose (associative) product is given by the rule

$$
\begin{gathered}
(a \otimes b \otimes h) \times\left(a^{\prime} \otimes b^{\prime} \otimes k\right)= \\
(-1)^{\operatorname{deg}(b) \cdot\left(\operatorname{deg}\left(a^{\prime}\right)+\operatorname{deg}\left(b^{\prime}\right)\right)} \times\left(a\left(h_{1} \rightarrow a^{\prime}\right)\right) \otimes\left(\left(S^{2 i}\left(h_{3}\right) \rightarrow b^{\prime}\right) b\right) \otimes h_{2} k .
\end{gathered}
$$

It is elementary although tedious to check that the product is indeed associative. Note that the product in $\Delta_{i}$ is determined by the three following properties

- the product is associative,

- $A^{e}$ is a dg subalgebra of $\Delta_{i}$ via the mapping $A^{e} \rightarrow A^{e} \otimes H, a \otimes b \mapsto a \otimes b \otimes 1$,

- $H$ is dg subalgebra of $\Delta_{i}$ via the mapping $H \rightarrow A^{e} \otimes H, h \mapsto 1 \otimes 1 \otimes h$,

- the product satisfies the following identity in $\Delta_{i}$

$$
h \times(a \otimes b)=\left(h_{1} \rightarrow a \otimes S^{2 i}\left(h_{3}\right) \rightarrow b\right) \times h_{2} .
$$

When $S$ is invertible, $\Delta_{i}$ features the following useful identity

$$
(a \otimes b) \times h=h_{2} \times\left(S^{-1}\left(h_{1}\right) \rightarrow a \otimes S^{2 i+1}\left(h_{3}\right) \rightarrow b\right) .
$$

In general, the following mapping is a homomorphism of $\mathrm{dg}$ algebras that makes of $\Lambda^{e}$ a left (and right) dg $\Delta_{i}$-module

$$
\begin{aligned}
\Delta_{i} & \rightarrow \Lambda^{e} \\
(a \otimes b) \times h & \mapsto(a \otimes b) \times\left(h_{1} \otimes S^{2 i+1}\left(h_{2}\right)\right) .
\end{aligned}
$$

The image of this mapping is the dg subalgebra of $\Lambda^{e}$ generated by $A^{e} \cup\left\{h_{1} \otimes\right.$ $\left.S^{2 i+1}\left(h_{2}\right) \mid h \in H\right\}$ because of the following identity in $\Lambda^{e}$,

$$
\left(h_{1} \otimes S^{2 i+1}\left(h_{2}\right)\right) \times(a \otimes b)=\left(h_{1} \rightarrow a \otimes S^{2 i}\left(h_{4}\right) \rightarrow b\right) \times\left(h_{2} \otimes S^{2 i+1}\left(h_{3}\right)\right) .
$$

When $S$ is invertible, this mapping is injective and has a retraction given by $a h \otimes$ $b k \mapsto a \otimes S^{-1}(k) \rightarrow b \otimes h$.

If $H$ is cocommutative, then $\Delta_{i}$ does not depend on $i$ and is isomorphic to a smash product of $A^{e} \sharp H$ (see [26, Remark 1.7]). This does not hold true in general.

The left $\mathrm{dg} \Delta_{i}$-modules are the $H_{S^{2 i}}$-equivariant left $\mathrm{dg} A$-bimodules defined in [30, Definition 2.2]. The latter are the left $\operatorname{dg} A$-bimodules $M$ endowed with a structure of left $H$-module (which preserves the degree and is compatible with the differential of the complex) in such a way that the following identity holds in $M$

$$
h \rightarrow(a m b)=\left(h_{1} \rightarrow a\right)\left(h_{2} \rightarrow m\right)\left(S^{2 i}\left(h_{3}\right) \rightarrow b\right) .
$$

In particular, $A \in \mathcal{C}\left(\Delta_{0}\right)$. 
Example. Let $A=\mathbb{k}\left[x_{1}, \ldots, x_{n}\right]$. Let $H=\mathcal{U}(\mathfrak{g})$ as in the example of 3.1. Let $\mathfrak{g} \rightarrow \operatorname{Der}_{\mathbb{k}}(A), X \mapsto \partial_{X}$ be a homomorphism of Lie algebras. Hence, $A$ is an $H$-module algebra. The structure of $\Delta_{0}$-module on $A^{e}$ is given by

$$
X \rightarrow(a \otimes b)=\partial_{X}(a) \otimes b+a \otimes \partial_{X}(b) .
$$

The sequence $\left(x_{i} \otimes 1-1 \otimes x_{i}\right)_{1 \leqslant i \leqslant n}$ of the commutative ring $A^{e}$ is regular and the quotient of $A^{e}$ by the ideal generated by this sequence is isomorphic to $A$. Recall that the Koszul resolution $\left(K^{\bullet}, d_{K}\right)$ of ${ }_{A} A_{A}$ is as follows. Denote $\oplus_{i=1}^{n} \mathbb{k} \cdot x_{i}$ by $V$. Let $K^{\bullet}$ be the graded vector space $\Lambda_{A^{e}}^{-\bullet}(A \otimes V \otimes A)$ concentrated in degrees $-n,-n+1, \ldots,-1,0$. This is a graded-commutative algebra over the commutative ring $A^{e}$ in the usual way (the product is denoted by $\wedge$ ). By a skew derivation of degree $\ell$ of $K^{\bullet}$ is meant a homogeneous $\mathbb{k}$-linear mapping $d: K^{\bullet} \rightarrow K^{\bullet+\ell}$ such that, for all homogeneous $\omega_{1}, \omega_{2} \in K^{\bullet}$,

$$
d\left(\omega_{1} \wedge \omega_{2}\right)=d\left(\omega_{1}\right) \wedge \omega_{2}+(-1)^{\ell \operatorname{deg}\left(\omega_{1}\right)} \omega_{1} \wedge d\left(\omega_{2}\right) .
$$

The skew derivations of degree 0 are usual $\mathbb{k}$-linear algebra derivations. Denote by $d_{K}$ the unique skew derivation of degree +1 of $K^{\bullet}$ such that $d_{K}(1 \otimes v \otimes 1)=$ $v \otimes 1-1 \otimes v \in A^{e}$ for all $v \in V$. Hence, $d_{K}$ is $A^{e}$-linear and squares to zero. By construction, $\left(K^{\bullet}, d_{K}\right)$ is the Koszul complex of the sequence $\left(x_{i} \otimes 1-1 \otimes x_{i}\right)_{1 \leqslant i \leqslant n}$ of the commutative ring $A^{e}$. This is a projective resolution of $A$ as an $A$-bimodule.

Here is how to endow $\left(K^{\bullet}, d_{K}\right)$ with an action of $H$ for which $K^{\bullet}$ lies in $\mathcal{C}\left(\Delta_{0}\right)$. Given any $m \in A$ written as a linear combination of monomials

$$
m=\lambda_{0}+\sum_{r \geqslant 1} \sum_{1 \leqslant j_{1} \leqslant \cdots \leqslant j_{r} \leqslant n} \lambda_{j_{1}, \ldots, j_{r}} x_{j_{1}} x_{j_{2}} \cdots x_{j_{r}},
$$

where $\lambda_{0}, \lambda_{j_{1}, \ldots, j_{r}} \in \mathbb{k}$, use the symbolic piece of notation

$$
\sum_{i} m_{i}^{\prime} \otimes x_{i} \otimes m_{i}^{\prime \prime}
$$

to denote

$$
\sum_{r \geqslant 1} \sum_{1 \leqslant j_{1} \leqslant \cdots \leqslant j_{r} \leqslant n} \lambda_{j_{1}, \ldots, j_{r}} \sum_{t=1}^{r} x_{j_{1}} \cdots x_{j_{t-1}} \otimes x_{j_{t}} \otimes x_{j_{t+1}} \cdots x_{j_{r}} \in A \otimes V \otimes A .
$$

For every $X \in \mathfrak{g}$, there exists a unique skew derivation of degree 0 of $K^{\bullet}$ denoted by $\partial_{X}$ and such that

(a) $\partial_{X}(a \otimes b)=X \rightarrow(a \otimes b)$ for all $a \otimes b \in A \otimes A$,

(b) and $\partial_{X}(1 \otimes v \otimes 1)=\sum_{i} \partial_{X}(v)_{i}^{\prime} \otimes x_{i} \otimes \partial_{X}(v)_{i}^{\prime \prime}$ for all $v \in V$.

Then, $d_{K} \circ \partial_{X}-\partial_{X} \circ d_{K}$ is a skew derivation of degree +1 of $K^{\bullet}$. Therefore, it vanishes on $K^{0}$. In view of (b), it vanishes on $1 \otimes V \otimes 1$. Thus, it is zero, that is, $\partial_{X}: K^{\bullet} \rightarrow K^{\bullet}$ is a morphism of complexes of vector spaces. The family $\left(\partial_{X}\right)_{X \in \mathfrak{g}}$ hence yields an action of $H$ on $K^{\bullet}$. In view of (a), $K^{\bullet}$ is a dg $\Delta_{0}$-module.

4.2. $A \sharp H$-bimodules arising from equivariant $A$-bimodules. Let $i \in \mathbb{Z}$ and assume that $S$ is invertible whenever $i<0$. Let $D$ be an $H_{S^{2 i}}$-equivariant $\operatorname{dg} A$ bimodule (equivalently, a left $\operatorname{dg} \Delta_{i}$-module). Let $\sigma \in \operatorname{Aut}_{\mathrm{k}-\text { alg }}(H)$ be such that the following identity holds in $H$

$$
\sigma(h)_{1} \otimes \sigma(h)_{2}=S^{2 i}\left(h_{1}\right) \otimes \sigma\left(h_{2}\right) .
$$

(for instance, $\sigma=S^{2 i} \circ \Xi_{\pi}^{r}$ for some algebra homomorphism $\pi: H \rightarrow \mathbb{k}$ ). When $S$ is invertible, this condition is equivalent to the following identity in $H$

$$
\sigma^{-1}(h)_{1} \otimes \sigma^{-1}(h)_{2}=S^{-2 i}\left(h_{1}\right) \otimes \sigma^{-1}\left(h_{2}\right) .
$$


4.2.1. According to [30, Lemma 2.5], the following actions endow $D \otimes H$ with a structure of $\operatorname{dg} \Lambda$-bimodule (see 4.1.0.4)

$$
\left\{\begin{array}{l}
a(d \otimes \ell) b=a d\left(\ell_{1} \rightarrow b\right) \otimes \ell_{2} \\
h(d \otimes \ell) k=h_{1} \rightarrow d \otimes \sigma\left(h_{2}\right) \ell k
\end{array}\right.
$$

In the sequel, it is denoted by $D \sharp{ }^{\sigma} H$. When $S$ is invertible, $D \sharp{ }^{\sigma} H$ features the following identity

$$
d \otimes \ell=\sigma^{-1}\left(\ell_{2}\right)\left(S^{-1-2 i}\left(\ell_{1}\right) \rightarrow d \otimes 1\right)
$$

In [29, Definition 3.2], the construction $D \sharp H$ is extended to $D \sharp X$, where $X$ is any complex of Hopf bimodules over $H$.

4.2.2. Assume that $S$ is invertible. The following lemma is a functorial interpretation of the previous construction. The mapping $\varphi: \Lambda \rightarrow \Lambda$ defined by $\varphi(a h)=$ $a \sigma\left(S^{-2 i}(h)\right)$ is an automorphism of the dg algebra $\Lambda$. Hence, $\Lambda \otimes{ }^{\varphi} \Lambda$ is a $\Lambda^{e}-\Delta_{i^{-}}$ bimodule. As a left $\operatorname{dg} \Lambda^{e}$-module it equals ${ }_{\Lambda} \Lambda \otimes \Lambda_{\Lambda}$. Its structure of right $\mathrm{dg}$ $\Delta_{i}$-module is given by $\left(\lambda \otimes \lambda^{\prime}\right) \cdot(a \otimes b \otimes h)= \pm \lambda a h_{1} \otimes \varphi\left(S^{2 i+1}\left(h_{2}\right) b\right) \lambda^{\prime}$ (where $\left.\lambda, \lambda^{\prime} \in \Lambda\right)$. In particular, $\left(\Lambda \otimes \otimes^{\varphi} \Lambda\right) \underset{\Delta_{i}}{\otimes D}$ inherits of a structure of $\operatorname{dg} \Lambda$-bimodule.

Lemma. Keep the setting introduced previously. Then, $D_{\sharp}^{\sigma} H \simeq\left(\Lambda \otimes{ }^{\varphi} \Lambda\right) \otimes D$ in $\mathcal{C}\left(\Lambda^{e}\right)$.

Proof. Denote $\left(\Lambda \otimes \otimes^{\varphi} \Lambda\right) \underset{\Delta_{i}}{\otimes} D$ by $\bar{D}$. Note the identity in $\bar{D}$

$$
(h \otimes k) \underset{\Delta_{i}}{\otimes} d=\left(1 \otimes \sigma\left(h_{2}\right) k\right) \underset{\Delta_{i}}{\otimes}\left(h_{1} \rightarrow d\right) .
$$

Indeed, the right hand-side term equals $\left(h_{1} \otimes \varphi\left(S^{2 i+1}\left(h_{2}\right)\right) \sigma\left(h_{3}\right) k\right) \underset{\Delta_{i}}{\otimes} d$; This is equal to $(h \otimes k) \otimes d$. Therefore, the linear mapping from $(\Lambda \otimes \Lambda) \otimes D$ to $D \otimes H$ defined by $(h a \otimes b k) \otimes d \mapsto \pm\left(h_{1} \rightarrow(a d b)\right) \otimes \sigma\left(h_{2}\right) k$ induces a linear mapping

$$
\begin{aligned}
\bar{D} & \rightarrow D \otimes H \\
(h a \otimes b k) \otimes d & \mapsto\left(h_{1} \rightarrow(a d b)\right) \otimes \sigma\left(h_{2}\right) k .
\end{aligned}
$$

Here, \pm is the $\operatorname{sign}(-1)^{\operatorname{deg}(b) \cdot \operatorname{deg}(d)}$. In view of 4.2.2.1), it is bijective and its inverse is the mapping defined by $d \otimes \ell \mapsto(1 \otimes \ell) \otimes d$. In order to prove the assertion of the lemma, it therefore suffices to prove that the latter mapping is a morphism of $\operatorname{dg} \Lambda$-bimodules from $D \sharp{ }^{\sigma} H$ to $\bar{D}$. This is done in the computations below made in $\bar{D}$ :

$$
\begin{aligned}
a\left((1 \otimes \ell) \underset{\Delta_{i}}{\otimes d} d\right) b & =(-1)^{\operatorname{deg}(b) \operatorname{deg}(d)}(a \otimes \ell b) \underset{\Delta_{i}}{\otimes} d \\
& =\left(1 \otimes \ell_{2}\right) \underset{\Delta_{i}}{\otimes} a d\left(\ell_{1}-b\right) .
\end{aligned}
$$

and

$$
\begin{aligned}
h\left((1 \otimes \ell) \underset{\Delta_{i}}{\otimes} d\right) k & =(h \otimes \ell k) \underset{\Delta_{i}}{\otimes d} \\
& =\left(1 \otimes \sigma\left(h_{2}\right) \ell k\right) \underset{\Delta_{i}}{\otimes}\left(h_{1} \rightarrow d\right) .
\end{aligned}
$$


4.2.3. Assume that $S$ is invertible and define a $\operatorname{dg} \Lambda$-bimodule $H^{\sigma^{-1}} \sharp D$ as follows. Its underlying complex of vector spaces is $H \otimes D$ and the actions of $\Lambda$ are given by

$$
\left\{\begin{aligned}
a(\ell \otimes d) b & =\ell_{2} \otimes\left(S^{-1}\left(\ell_{1}\right) \rightarrow a\right) d b \\
h(\ell \otimes d) k & =h \ell \sigma^{-1}\left(k_{2}\right) \otimes S^{-2 i-1}\left(k_{1}\right) \rightarrow d .
\end{aligned}\right.
$$

Lemma. Keep the setting introduced previously. The following mapping is an isomorphism in $\mathcal{C}\left(\Lambda^{e}\right)$

$$
\begin{aligned}
D \sharp{ }^{\sigma} H & \rightarrow H^{\sigma^{-1} \sharp D} \\
d \otimes \ell & \mapsto \sigma^{-1}\left(\ell_{2}\right) \otimes S^{-2 i-1}\left(\ell_{1}\right) \rightarrow d .
\end{aligned}
$$

Proof. Denote this mapping by $\varphi$. Consider the mapping $H^{\sigma^{-1}} \sharp D \rightarrow D \sharp{ }^{\sigma} H$ defined by $\ell \otimes d \mapsto \ell_{1} \rightarrow d \otimes \sigma\left(\ell_{2}\right)$. In view of 4.2.0.1), 4.2.0.2 and 4.2.1.2), it is an inverse of $\varphi$. That $\varphi$ is a morphism in $\mathcal{C}\left(\Lambda^{e}\right)$ follows from the computations below

$$
\begin{aligned}
\varphi(h(d \otimes \ell) k) & =\varphi\left(h_{1} \rightarrow d \otimes \sigma\left(h_{2}\right) \ell k\right) \\
& =h_{3} \sigma^{-1}\left(\ell_{2}\right) \sigma^{-1}\left(k_{2}\right) \otimes\left(S^{-2 i-1}\left(k_{1}\right) S^{-2 i-1}\left(\ell_{1}\right) S^{-1}\left(h_{2}\right) h_{1}\right) \rightarrow d \\
& =h \varphi(d \otimes \ell) k
\end{aligned}
$$

and

$$
\begin{aligned}
\varphi(a(d \otimes \ell) b)= & \varphi\left(a d\left(\ell_{1} \rightarrow b\right) \otimes \ell_{2}\right) \\
= & \sigma^{-1}\left(\ell_{3}\right) \otimes S^{-2 i-1}\left(\ell_{2}\right) \rightarrow\left(a d\left(\ell_{1} \rightarrow b\right)\right) \\
= & \sigma^{-1}\left(\ell_{5}\right) \otimes \\
& \left(S^{-2 i-1}\left(\ell_{4}\right) \rightarrow a\right)\left(S^{-2 i-1}\left(\ell_{3}\right) \rightarrow d\right)\left(\left(S^{-1}\left(\ell_{2}\right) \ell_{1}\right) \rightarrow b\right) \\
= & a\left(\sigma^{-1}\left(\ell_{2}\right) \otimes S^{-2 i-1}\left(\ell_{1}\right) \rightarrow d\right) b .
\end{aligned}
$$

4.3. Morphisms defined on equivariant $\mathrm{dg}$ bimodules. The following result is used in the description of $\operatorname{RHom}_{\Lambda^{e}}\left(\Lambda, \Lambda^{e}\right)$ in Section 5 and in the description of (deformed) Calabi-Yau completions of $A \sharp H$ in Section 6. Note that,

- when $i=1$, part (1) is being considered in [26, (1.3)],

- when $i=0$, the definition of $h \rightarrow f$ in part (2) coincides with the one of $S^{-2}(h) \rightarrow f$ in [26, (1.2)] and with the one of $f S^{-1}(h)$ in [29, Definition $4.1]$,

Lemma. Let $i \in \mathbb{Z}$ and assume that $S$ is invertible. Let $X \in \mathcal{C}\left(\Delta_{i}\right), U \in \mathcal{C}\left(\Delta_{1-i}\right)$, and $M \in \mathcal{C}\left(\Lambda^{e}\right)$.

(1) There exists a structure of left $d g H$-module on $U \underset{A^{e}}{\otimes} M$ such that

$$
h \rightarrow(u \otimes m)=\left(h_{2} \rightarrow u\right) \otimes S^{2-2 i}\left(h_{3}\right) m S\left(h_{1}\right) .
$$

(2) There is a structure of left $d g H$-module on $\operatorname{Hom}_{A^{e}}(X, M)$ such that

$$
(h \rightarrow f)(x)=S^{2-2 i}\left(h_{3}\right) f\left(S^{1-2 i}\left(h_{2}\right) \rightarrow x\right) S\left(h_{1}\right) .
$$

(3) Given $f \in \operatorname{Hom}_{A^{e}}(X, M)$, the following are equivalent

(i) $f \in \operatorname{Hom}_{\Delta_{i}}(X, M)$,

(ii) $(\forall h \in H) h \rightarrow f=\epsilon(h) f$,

(iii) $(\forall h \in H)(\forall x \in X) \quad f(h \rightarrow x)=h_{1} f(x) S^{1+2 i}\left(h_{2}\right)$.

(4) There is a functorial isomorphism

$$
\begin{aligned}
\operatorname{Hom}_{H}\left({ }_{H} \mathbb{k}, \operatorname{Hom}_{A^{e}}(X, M)\right) & \stackrel{\simeq}{ } \operatorname{Hom}_{\Delta_{i}}(X, M) \\
\lambda & \mapsto \lambda(1) .
\end{aligned}
$$

(5) Assume, here, that $M$ has an additional structure of right $d g A^{e}$-module which is compatible with the structure of left $d g \Lambda^{e}$-module in the sense of 2.2.2.4) (e.g. $\left.M=A^{e}\right)$. Consider $\operatorname{Hom}_{A^{e}}(X, M)$ as $d g$ A-bimodule using this additional action of $A^{e}$ on $M$. Then (2) makes of $\operatorname{Hom}_{A^{e}}(X, M)$ an $H_{S^{2-2 i} \text {-equivariant }} d g$ A-bimodule: $\operatorname{Hom}_{A^{e}}(X, M) \in \mathcal{C}\left(\Delta_{1-i}\right)$. 
(6) The canonical mapping $\operatorname{Hom}_{A^{e}}\left(X, A^{e}\right) \underset{A^{e}}{\otimes} M \rightarrow \operatorname{Hom}_{A^{e}}(X, M)$ is H-linear.

Proof. (1) Here, $U$ is considered as a right $\operatorname{dg} A^{e}$-module in a natural way: $u<$ $(a \otimes b)=(-1)^{\operatorname{deg}(b)(\operatorname{deg}(a u))} b u a$. The identity given in the statement endows $U \otimes M$ with a structure of left $\mathrm{dg} H$-module. Using that $U$ is $H_{S^{2-2 i}}$-equivariant, a simple computation shows that this structure factors through $U \otimes M \rightarrow U \otimes A^{e} U$.

(2) The given action makes of $\operatorname{Hom}_{\mathbb{k}}(X, M)$ a left $\mathrm{dg} H$-module. The following computation where $f \in \operatorname{Hom}_{A^{e}}(X, M)$ proves that $\operatorname{Hom}_{A^{e}}(X, M)$ is a $\operatorname{dg} H$ submodule of $\operatorname{Hom}_{\mathbb{k}}(X, M)$

$$
\begin{aligned}
(h \rightarrow f)(a x b) & =S^{2-2 i}\left(h_{3}\right) f\left(S^{1-2 i}\left(h_{2}\right) \rightarrow(a x b)\right) S\left(h_{1}\right) \\
& = \pm S^{2-2 i}\left(h_{5}\right) S^{1-2 i}\left(h_{4}\right) \rightarrow a f\left(S^{1-2 i}\left(h_{3}\right) \rightarrow x\right) S\left(h_{2}\right) \rightarrow b S\left(h_{1}\right) \\
& = \pm a(h \rightarrow f)(x) b
\end{aligned}
$$

where \pm is the $\operatorname{sign}(-1)^{\operatorname{deg}(f) \cdot \operatorname{deg}(a)}$.

(3) The equivalence " $(i) \Leftrightarrow($ iii $)$ " follows from the definition of $\Delta_{i}$ (see 4.1.0.3). The implication " $($ iii $) \Rightarrow($ ii $)$ " follows from the definition of the action of $H$ on $\operatorname{Hom}_{A^{e}}(X, M)$. If $(i i)$ holds true, then

$$
\begin{aligned}
f(h \rightarrow x) & =\epsilon\left(h_{1}\right) f\left(h_{2} \rightarrow x\right) \epsilon\left(h_{3}\right) \\
& =h_{1} S\left(h_{2}\right) f\left(h_{3} \rightarrow x\right) S^{2 i}\left(h_{4}\right) S^{1+2 i}\left(h_{5}\right) \\
& =h_{1}\left(S^{2 i-1}\left(h_{2}\right) \rightarrow f\right)(x) S^{1+2 i}\left(h_{3}\right) \\
& =h_{1} f(x) S^{1+2 i}\left(h_{2}\right) .
\end{aligned}
$$

This proves that $(i i) \Rightarrow(i i i)$.

(4) In view of (3), the given mapping is well-defined and surjective. The mapping is injective, and hence it is an isomorphism.

(5) This follows from the computation below where $f \in \operatorname{Hom}_{A^{e}}(X, M)$. To avoid any confusion, note that the computation does not involve the action of $A^{e}$ on $M$ arising from the action of $\Lambda^{e}$, and that the third equality is due to 2.2.2.4.

$$
\begin{array}{ll}
(h \rightarrow(f \leftarrow(a \otimes b)))(x) & = \\
\left(S^{2-2 i}\left(h_{3}\right) \otimes S\left(h_{1}\right)\right) \rightarrow\left((f<(a \otimes b))\left(S^{1-2 i}\left(h_{2}\right) \rightarrow x\right)\right) & = \\
\pm\left(S^{2-2 i}\left(h_{3}\right) \otimes S\left(h_{1}\right)\right) \rightarrow\left(f\left(S^{1-2 i}\left(h_{2}\right) \rightarrow x\right) \leftarrow(a \otimes b)\right) & = \\
\pm\left(\left(S^{2-2 i}\left(h_{4}\right) \otimes S\left(h_{2}\right)\right) \rightarrow\left(f\left(S^{1-2 i}\left(h_{3}\right) \rightarrow x\right)\right)\right) \leftarrow & \\
\quad\left(\left(S^{2-2 i}\left(h_{5}\right) \otimes S\left(h_{1}\right)\right) \rightarrow(a \otimes b)\right) & = \\
\pm\left(\left(S^{2-2 i}\left(h_{4}\right) \otimes S\left(h_{2}\right)\right) \rightarrow f\left(S^{1-2 i}\left(h_{3}\right) \rightarrow x\right)\right) & \\
\quad \leftarrow\left(S^{2-2 i}\left(h_{5}\right) \rightarrow a \otimes h_{1} \rightarrow b\right) & = \\
\left(\left(h_{2} \rightarrow f\right) \leftarrow\left(S^{2-2 i}\left(h_{3}\right) \rightarrow a \otimes h_{1} \rightarrow b\right)\right)(x), &
\end{array}
$$

where \pm is the $\operatorname{sign}(-1)^{\operatorname{deg}(x) \cdot(\operatorname{deg}(a)+\operatorname{deg}(b))}$. In other words, using bimodule notation, then

$$
h \rightarrow(a f b)=\left(h_{1} \rightarrow a\right)\left(h_{2} \rightarrow f\right)\left(S^{2-2 i}\left(h_{3}\right) \rightarrow b\right) .
$$

(6) Following (5), $\operatorname{Hom}_{A^{e}}\left(X, A^{e}\right)$ is a left $\mathrm{dg} \Delta_{1-i}$-module for which the corresponding structure of right dg $A^{e}$-module is given by $(f \leftarrow(a \otimes b))(x)=f(x) \times(a \otimes$ $b)$. Hence, (1) provides $\operatorname{Hom}_{A^{e}}\left(X, A^{e}\right) \underset{A^{e}}{\otimes} M$ with a structure of left $\operatorname{dg} H$-module.

Let $\varphi \in \operatorname{Hom}_{A^{e}}\left(X, A^{e}\right), m \in M$ and $h \in H$; Denote by $f$ the image of $\varphi \otimes m$ in $\operatorname{Hom}_{A^{e}}(X, M)$; It is defined by $f(x)=(-1)^{\operatorname{deg}(m) \cdot \operatorname{deg}(x)} \varphi(x) \rightarrow m$ (where $\rightarrow$ is used to denote the action of $\Lambda^{e}$ on $\left.M\right)$; Denote by $g$ the image of $h \rightarrow(\varphi \otimes$ $m)$ in $\operatorname{Hom}_{A^{e}}(X, M)$; It is defined by $g(x)=(-1)^{\operatorname{deg}(m) \cdot \operatorname{deg}(x)}\left(h_{2} \rightarrow \varphi\right)(x) \rightarrow$ $\left(S^{2-2 i}\left(h_{3}\right) m S\left(h_{1}\right)\right)$. Then $g=h \rightarrow f$ according to the following computation 
where $M$ is considered alternatively as a left $\operatorname{dg} \Lambda^{e}$-module and as a $\operatorname{dg} \Lambda$-bimodule

$$
\begin{array}{rlll}
(h \rightarrow f)(x) & = & \left(S^{2-2 i}\left(h_{3}\right) \otimes S\left(h_{1}\right)\right) \rightarrow f\left(S^{1-2 i}\left(h_{2}\right) \rightarrow x\right) \\
& = & \pm\left(S^{2-2 i}\left(h_{3}\right) \otimes S\left(h_{1}\right)\right) \rightarrow\left(\varphi\left(S^{1-2 i}\left(h_{2}\right) \rightarrow x\right) \rightarrow m\right) \\
= & \pm\left(\left(S^{2-2 i}\left(h_{4}\right) \otimes S\left(h_{2}\right)\right) \rightarrow \varphi\left(S^{1-2 i}\left(h_{3}\right) \rightarrow x\right)\right) \\
2.2 .2 .3 & & \rightarrow\left(\left(S^{2-2 i}\left(h_{5}\right) \otimes S\left(h_{1}\right)\right) \rightarrow m\right) \\
& = & \pm\left(h_{2} \rightarrow \varphi\right)(x) \rightarrow\left(S^{2-2 i}\left(h_{3}\right) m S\left(h_{1}\right)\right) \\
= & g(x) .
\end{array}
$$

4.4. Equivariant actions on duals over $A$. In this section, $S$ is assumed to be invertible and $A$ is assumed to be a $\mathbb{k}$-algebra. Let $i \in \mathbb{Z}$, let $D$ be an $H_{S^{2 i}-}$ equivariant $A$-bimodule. Let $\sigma \in \operatorname{Aut}_{\mathrm{k}-\text { alg }}(H)$ be such that the identity $\sigma(h)_{1} \otimes$ $\sigma(h)_{2}=S^{2 i}\left(h_{1}\right) \otimes \sigma\left(h_{2}\right)$ holds in $H$. When $D$ is invertible as an $A$-bimodule, then $\operatorname{Hom}_{A}(D, A) \simeq \operatorname{Hom}_{A^{\text {op }}}(D, A)$ is the inverse of $D$. Hence, this section considers $\Lambda$-bimodules arising from $\operatorname{Hom}_{A}(D, A)$ and $\operatorname{Hom}_{A^{\mathrm{op}}}(D, A)$.

4.4.1. The following result shows that $\operatorname{Hom}_{A}(D, A)$ and $\operatorname{Hom}_{A^{\mathrm{op}}}(D, A)$ both have

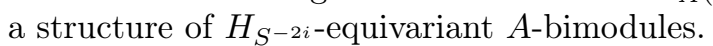

Lemma. Keep the setting stated previously.

(1) There exists a structure of left $H$-module on $\operatorname{Hom}_{A}(D, A)$ such that $(h \rightarrow$ $f)(d)=S^{-2 i}\left(h_{2}\right) \rightarrow f\left(S^{-1-2 i}\left(h_{1}\right) \rightarrow d\right)$ for every $f \in \operatorname{Hom}_{A}(D, A)$. For this structure, $\operatorname{Hom}_{A}(D, A)$ is an $H_{S^{-2 i}}$-equivariant $A$-bimodule.

(2) There exists a structure of left $H$-module on $\operatorname{Hom}_{A^{\mathrm{op}}}(D, A)$ such that $(h \rightarrow$ $f)(d)=h_{1} \rightarrow f\left(S^{1-2 i}\left(h_{2}\right) \rightarrow d\right)$ for every $f \in \operatorname{Hom}_{A^{\mathrm{op}}}(D, A)$. For this

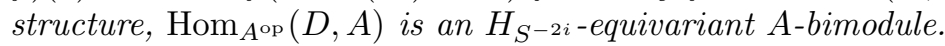

Proof. (1) If $f \in \operatorname{Hom}_{A}(D, A)$, then the mapping $h \rightarrow f: D \rightarrow A$ introduced in the statement is $A$-linear, indeed

$$
\begin{aligned}
(h \rightarrow f)(a d) & =S^{-2 i}\left(h_{2}\right) \rightarrow f\left(S^{-1-2 i}\left(h_{1}\right) \rightarrow(a d)\right) \\
& =S^{-2 i}\left(h_{3}\right) \rightarrow\left(\left(S^{-1-2 i}\left(h_{2}\right) \rightarrow a\right) f\left(S^{-1-2 i}\left(h_{1}\right) \rightarrow d\right)\right) \\
& =a(h \rightarrow f)(d) .
\end{aligned}
$$

Note that the $A$-bimodule structure of $\operatorname{Hom}_{A}(D, A)$ is such that $(a f b)(d)=f(d a) b$. The following computation shows the second assertion of (1)

$$
\begin{aligned}
& (h \rightarrow(a f b))(d) \quad=\quad S^{-2 i}\left(h_{2}\right) \rightarrow\left(f\left(\left(S^{-1-2 i}\left(h_{1}\right) \rightarrow d\right) a\right) b\right) \\
& \begin{array}{ll}
\underset{4.1 .0 .2}{=} & S^{-2 i}\left(h_{3}\right) \rightarrow\left(f\left(S^{-1-2 i}\left(h_{2}\right) \rightarrow\left(d\left(h_{1} \rightarrow a\right)\right)\right) b\right) \\
= & \left(h_{2} \rightarrow f\right)\left(d\left(h_{1} \rightarrow a\right)\right)\left(S^{-2 i}\left(h_{3}\right) \rightarrow b\right)
\end{array} \\
& =\left(\left(h_{1} \rightarrow a\right)\left(h_{2} \rightarrow f\right)\left(S^{-2 i}\left(h_{3}\right) \rightarrow b\right)\right)(d) \text {. }
\end{aligned}
$$

(2) is proved similarly.

4.4.2. The following technical result is used later to prove that $D$ is invertible as an $A$-bimodule if and only if $D \sharp^{\sigma} H$ is invertible as a $\Lambda$-bimodule.

Lemma. Keep the setting stated previously. Let $M \in \bmod (\Lambda)$. Let $N \in \bmod \left(\Lambda^{\mathrm{op}}\right)$.

(1) The following endows $\operatorname{Hom}_{A}(D, M)$ with a structure of left $\Lambda$-module (here $\left.g \in \operatorname{Hom}_{A}(D, M)\right)$

$$
\begin{aligned}
a g: \quad d & \mapsto g(d a) \\
h g: \quad d & \mapsto \sigma^{-1}\left(h_{2}\right) g\left(S^{-2 i-1}\left(h_{1}\right) \rightarrow d\right) .
\end{aligned}
$$

For this structure, the following mapping is an isomorphism in $\bmod (\Lambda)$

$$
\begin{aligned}
\operatorname{Hom}_{\Lambda}\left(D \sharp^{\sigma} H, M\right) & \rightarrow \operatorname{Hom}_{A}(D, M) \\
f & \mapsto f(-\otimes 1) .
\end{aligned}
$$


(2) Assume that $M=\Lambda$. Then, the canonical mapping from $\operatorname{Hom}_{A}(D, A) \otimes H$ to $\operatorname{Hom}_{A}(D, A \otimes H)$ is a morphism in $\bmod \left(\Lambda^{e}\right)$ from $\operatorname{Hom}_{A}(D, A) \sharp^{\sigma^{-1}} H$ to $\operatorname{Hom}_{A}(D, \Lambda)$. In particular, if $D$ is finitely presented as a left $A$-module or if $\operatorname{dim}_{\mathbb{k}} H<\infty$, then $\operatorname{Hom}_{\Lambda}(D \sharp \sigma H, \Lambda) \simeq \operatorname{Hom}_{A}(D, A) \sharp^{\sigma^{-1}} H$ in $\bmod \left(\Lambda^{e}\right)$.

(3) The following actions endow $\operatorname{Hom}_{A^{\mathrm{op}}}(D, N)$ with a structure of right $\Lambda$ module (here $g \in \operatorname{Hom}_{A \text { op }}(D, N)$ )

$$
\begin{aligned}
& g a: d \mapsto g(a d) \\
& g h: \quad d \mapsto g\left(h_{1} \rightarrow d\right) \sigma\left(h_{2}\right) .
\end{aligned}
$$

For this structure, the following mapping is an isomorphism in $\bmod \left(\Lambda^{\mathrm{op}}\right)$

$$
\begin{aligned}
\operatorname{Hom}_{\Lambda^{\mathrm{op}}}\left(D \sharp^{\sigma} H, N\right) & \rightarrow \operatorname{Hom}_{A^{\mathrm{op}}}(D, N) \\
f & \mapsto f(-\otimes 1) .
\end{aligned}
$$

(4) Assume that $N=\Lambda$. Then, there is a morphism in $\bmod \left(\Lambda^{e}\right)$

$$
\operatorname{Hom}_{A^{\mathrm{op}}}(D, A) \sharp^{\sigma^{-1}} H \rightarrow \operatorname{Hom}_{A^{\mathrm{op}}}(D, \Lambda)
$$

such that the image of a tensor $\varphi \otimes \ell$ is the morphism $D \rightarrow \Lambda$ given by $d \mapsto \varphi\left(\ell_{1} \rightarrow d\right) \sigma\left(\ell_{2}\right)$. If $D$ is finitely presented as a right $A$-module or if $\operatorname{dim}_{\mathbb{k}} H<\infty$, then it is an isomorphism.

Proof. (1) Given $f \in \operatorname{Hom}_{\Lambda}\left(D \sharp{ }^{\sigma} H, M\right)$, consider $g: D \rightarrow M$ defined by $g(d)=$ $f(d \otimes 1)$. Then,

- $g \in \operatorname{Hom}_{A}(D, M)$,

- $f$ is given by $f(d \otimes \ell)=\sigma^{-1}\left(\ell_{2}\right) g\left(S^{-1-2 i}\left(\ell_{1}\right) \rightarrow d\right)$ (see 4.2.1.2)).

Under the above construction, given $a \in A$ and $h \in H$, the morphisms af, $h f \in$ $\operatorname{Hom}_{\Lambda}\left(D \sharp^{\sigma} H, M\right)$ are mapped onto $a g$ and $h g$, respectively.

Conversely, let $g \in \operatorname{Hom}_{A}(D, M)$ and define $f: D \otimes H \rightarrow M$ by $f(d \otimes \ell)=$ $\sigma^{-1}\left(\ell_{2}\right) g\left(S^{-1-2 i}\left(\ell_{1}\right) \rightarrow d\right)$. The following computations made in $M$ show that $f \in \operatorname{Hom}_{\Lambda}\left(D \sharp^{\sigma} H, M\right)$

$$
\begin{aligned}
f(a(d \otimes \ell)) & =f(a d \otimes \ell) \\
& =\sigma^{-1}\left(\ell_{2}\right) g\left(S^{-1-2 i}\left(\ell_{1}\right) \rightarrow(a d)\right) \\
& =\sigma^{-1}\left(\ell_{3}\right)\left(S^{-1-2 i}\left(\ell_{2}\right) \rightarrow a\right) g\left(S^{-1-2 i}\left(\ell_{1}\right) \rightarrow d\right) \\
& =\sigma^{-1}\left(\ell_{2}\right)_{2}\left(S^{-1}\left(\sigma^{-1}\left(\ell_{2}\right)_{1}\right) \rightarrow a\right) g\left(S^{-1-2 i}\left(\ell_{1}\right) \rightarrow d\right) \\
& =a \sigma^{-1}\left(\ell_{2}\right) g\left(S^{-1-2 i}\left(\ell_{1}\right) \rightarrow d\right) \\
& =a f(d \otimes \ell)
\end{aligned}
$$

and

$$
\begin{aligned}
f(h(d \otimes \ell)) & =f\left(h_{1} \rightarrow d \otimes \sigma\left(h_{2}\right) \ell\right) \\
& =\sigma^{-1}\left(\sigma\left(h_{3}\right) \ell_{2}\right) g\left(S^{-1-2 i}\left(S^{2 i}\left(h_{2}\right) \ell_{1}\right) \rightarrow\left(h_{1} \rightarrow d\right)\right) \\
& =h \sigma^{-1}\left(\ell_{2}\right) g\left(S^{-1-2 i}\left(\ell_{1}\right) \rightarrow d\right) \\
& =h f(d \otimes \ell) .
\end{aligned}
$$

The previous considerations prove that the mapping from $\operatorname{Hom}_{\Lambda}(D \sharp \sigma H, M)$ to $\operatorname{Hom}_{A}(D, M)$ given in the statement of the lemma is well-defined and bijective. They also prove that the actions of $A$ and $H$ on $\operatorname{Hom}_{A}(D, M)$ given in the statement form a structure of $\Lambda$-module such that the mapping mentioned previously if $\Lambda$ linear.

(2) It suffices to prove the first statement. The structure of left $H$-module on $\operatorname{Hom}_{A}(D, M)$ is functorial in $M$, and hence, $\operatorname{Hom}_{A}(D, \Lambda)$ is a $\Lambda$-bimodule with structure of right $\Lambda$-module inherited from the one of $\Lambda$. The given canonical mapping is a morphism in $\bmod (A)$ and $\bmod \left(H^{\mathrm{op}}\right)$. There remains to prove that it is so in $\bmod \left(A^{\mathrm{op}}\right)$ and in $\bmod (H)$. Let $\varphi \otimes \ell \in \operatorname{Hom}_{A}(D, A) \otimes H$, and denote by $f: d \mapsto \varphi(d) \ell$ its image in $\operatorname{Hom}_{A}(D, \Lambda)$. 
Let $a \in A$, and denote by $f^{\prime}$ the image of $(\varphi \otimes \ell) a=\left(\varphi\left(\ell_{1} \rightarrow a\right) \otimes \ell_{2}\right)$ in $\operatorname{Hom}_{A}(D, \Lambda)$. The computation below made in $\Lambda$ proves that $f^{\prime}=f a$

$$
\begin{aligned}
(f a)(d) & =f(d) a \\
& =\varphi(d) \ell a \\
& =\varphi(d)\left(\ell_{1} \rightarrow a\right) \ell_{2} \\
& =f^{\prime}(d) .
\end{aligned}
$$

Let $h \in H$, and denote by $f^{\prime \prime}$ the image of $h(\varphi \otimes \ell)=h_{1} \rightarrow \varphi \otimes \sigma^{-1}\left(h_{2}\right) \ell$ in $\operatorname{Hom}_{\Lambda}(D, \Lambda)$. The computation below made in $\Lambda$ proves that $f^{\prime \prime}=h f$

$$
\begin{aligned}
& (h f)(d) \underset{\text { see }(1)}{=} \sigma^{-1}\left(h_{2}\right) f\left(S^{-1-2 i}\left(h_{1}\right) \rightarrow d\right) \\
& =\sigma^{-1}\left(h_{2}\right) \varphi\left(S^{-1-2 i}\left(h_{1}\right) \rightarrow d\right) \ell \\
& =S^{-2 i}\left(h_{2}\right) \rightarrow \varphi\left(S^{-1-2 i}\left(h_{1}\right) \rightarrow d\right) \sigma^{-1}\left(h_{3}\right) \ell \\
& =\left(h_{1} \rightarrow \varphi\right)(d) \sigma^{-1}\left(h_{2}\right) \ell \\
& =f^{\prime \prime}(d) \text {. }
\end{aligned}
$$

This proves (2).

(3) Let $f \in \operatorname{Hom}_{\Lambda} \mathrm{op}(\Lambda, N)$ and define $g \in \operatorname{Hom}_{\mathbb{k}}(D, N)$ by $g(d)=f(d \otimes 1)$. Then, $g \in \operatorname{Hom}_{A^{\mathrm{op}}}(D, N)$. This construction defines a mapping from $\operatorname{Hom}_{\Lambda^{\mathrm{op}}}\left(D \sharp{ }^{\sigma} H, N\right)$ to $\operatorname{Hom}_{A^{\text {op }}}(D, N)$. Keep $f$ and $g$ as above. Given $a \in A$, then $g a$ corresponds to $f a$ under the same construction. Also, given $h \in H$, then $g h$ corresponds to $f h$ under this construction as proved by the following computation

$$
\begin{aligned}
(f h)(d \otimes 1) & =f(h(d \otimes 1)) \\
& =f\left(h_{1} \rightarrow d \otimes \sigma\left(h_{2}\right)\right) \\
& =g\left(h_{1} \rightarrow d\right) \sigma\left(h_{2}\right) .
\end{aligned}
$$

Now, let $g \in \operatorname{Hom}_{A^{\text {op }}}(D, N)$ and define $f: D \sharp{ }^{\sigma} H \rightarrow N$ by $f(d \otimes \ell)=g(d) \ell$. Then, $f$ lies in $\operatorname{Hom}_{H^{\mathrm{op}}}\left(D \sharp{ }^{\sigma} H, N\right)$. And so does it in $\operatorname{Hom}_{A^{\mathrm{op}}}\left(D \sharp{ }^{\sigma} H, N\right)$ as proved by the following computation

$$
\begin{aligned}
f((d \otimes \ell) a) & =f\left(d\left(\ell_{1} \rightarrow a\right) \otimes \ell_{2}\right) \\
& =g\left(d\left(\ell_{1} \rightarrow a\right)\right) \ell_{2} \\
& =g(d)\left(\ell_{1} \rightarrow a\right) \ell_{2} \\
& =g(d) \ell a \\
& =f(d \otimes \ell) a .
\end{aligned}
$$

These considerations prove that there is a well-defined mapping from $\operatorname{Hom}_{A^{\mathrm{op}}}(D, N)$ to $\operatorname{Hom}_{\Lambda^{\mathrm{op}}}\left(D \sharp{ }^{\sigma} H, N\right)$ which is inverse to the mapping from $\operatorname{Hom}_{\Lambda^{\mathrm{op}}}\left(D \sharp{ }^{\sigma} H, N\right)$ to $\operatorname{Hom}_{A^{\text {op }}}(D, N)$ introduced in the statement of $(3)$. This proves $(3)$.

(4) Like in $(2), \operatorname{Hom}_{A \text { op }}(D, \Lambda)$ is a $\Lambda$-bimodule with structure of left $\Lambda$-module inherited from the one of $\Lambda$.

First, the given mapping from $\operatorname{Hom}_{A^{\text {op }}}(D, A) \sharp \sigma^{-1} H$ to $\operatorname{Hom}_{A^{\text {op }}}(D, \Lambda)$ is welldefined. Indeed, let $\varphi \otimes \ell \in \operatorname{Hom}_{A^{\mathrm{op}}}(D, A) \sharp^{\sigma^{-1} H}$. Define a mapping $g: D \rightarrow \Lambda$ by $g(d)=\varphi\left(\ell_{1} \rightarrow d\right) \sigma\left(\ell_{2}\right)$. In view of the following equalities in $\Lambda$, this mapping is a morphism in $\bmod \left(A^{\text {op }}\right)$

$$
\begin{aligned}
g(d a) & =\varphi\left(\ell_{1} \rightarrow(d a)\right) \sigma\left(\ell_{2}\right) \\
& =\varphi\left(\ell_{1} \rightarrow d\right)\left(S^{2 i}\left(\ell_{2}\right) \rightarrow a\right) \sigma\left(\ell_{3}\right) \\
& =\varphi\left(\ell_{1} \rightarrow d\right) \sigma\left(\ell_{2}\right) a .
\end{aligned}
$$

Denote by $\theta$ the resulting mapping from $\operatorname{Hom}_{A^{\mathrm{op}}}(D, A) \sharp \sigma^{-1} H$ to $\operatorname{Hom}_{A^{\text {op }}}(D, \Lambda)$. 
Next, $\theta$ is a morphism in $\bmod \left(\Lambda^{e}\right)$. Indeed, keep the notation $(\varphi, \ell, g)$ introduced previously, then

$$
\begin{aligned}
(a g)(d) & =a g(d) \\
& =(a \varphi)\left(\ell_{1} \rightarrow d\right) \sigma\left(\ell_{2}\right) \\
(h g)(d) & =h g(d) \\
& =h \varphi\left(\ell_{1} \rightarrow d\right) \sigma\left(\ell_{2}\right) \\
& =h_{1} \rightarrow \varphi\left(\ell_{1} \rightarrow d\right) h_{2} \sigma\left(\ell_{2}\right) \\
& =h_{1} \rightarrow \varphi\left(\left(S^{1-2 i}\left(h_{2}\right) S^{-2 i}\left(h_{3}\right) \ell_{1}\right) \rightarrow d\right) h_{4} \sigma\left(\ell_{2}\right) \\
& =\left(h_{1} \rightarrow \varphi\right)\left(\left(\sigma^{-1}\left(h_{2}\right) \ell_{1} \rightarrow d\right) \sigma\left(\left(\sigma^{-1}\left(h_{2}\right) \ell\right)_{2}\right)\right. \\
& \\
(g a)(d) & =g(a d) \\
& =\varphi\left(\ell_{1} \rightarrow(a d)\right) \sigma\left(\ell_{2}\right) \\
& =\left(\varphi \cdot\left(\ell_{1} \rightarrow a\right)\right)\left(\ell_{2} \rightarrow d\right) \sigma\left(\ell_{3}\right) \\
& =g\left(h_{1} \rightarrow d\right) \sigma\left(h_{2}\right) \\
(g h)(d) & =\left(\left(\ell_{1} h_{1}\right) \rightarrow d\right) \sigma\left(\ell_{2} h_{2}\right) .
\end{aligned}
$$

In order to prove (4), there only remains to prove that $\theta$ is bijective when $D$ is finitely presented as a right $A$-module or $\operatorname{dim}_{\mathbb{k}} H<\infty$. For this purpose, consider the composite mapping

$$
H^{\sigma} \sharp \operatorname{Hom}_{A^{\text {op }}}(D, A) \underset{\left[\frac{\sim}{4.2 .3}\right.}{\stackrel{\sim}{\longrightarrow}} \operatorname{Hom}_{A^{\text {op }}}(D, A) \sharp{ }^{\sigma^{-1}} H \stackrel{\theta}{\rightarrow} \operatorname{Hom}_{A^{\mathrm{op}}}(D, \Lambda) .
$$

Recall from the proof in 4.2 .3 that the left hand-side mapping is defined by $\ell \otimes \varphi \mapsto$ $\ell_{1} \rightarrow \varphi \otimes \sigma^{-1}\left(\ell_{2}\right)$. Hence, to any $\ell \otimes \varphi \in H \otimes \operatorname{Hom}_{A^{\text {op }}}(D, A)$, the composite mapping associates $\theta\left(\ell_{1} \rightarrow \varphi \otimes \sigma^{-1}\left(\ell_{2}\right)\right)$. Denote this morphism by $g$. Then

$$
\begin{aligned}
g(d) & =\left(\ell_{1} \rightarrow \varphi\right)\left(\sigma^{-1}\left(\ell_{2}\right)_{1} \rightarrow d\right) \sigma\left(\sigma^{-1}\left(\ell_{2}\right)_{2}\right) \\
& =\left(\ell_{1} \rightarrow \varphi\right)\left(S^{-2 i}\left(\ell_{2}\right) \rightarrow d\right) \ell_{3} \\
& =\ell_{1} \rightarrow \varphi\left(\left(S^{1-2 i}\left(\ell_{2}\right) S^{-2 i}\left(\ell_{3}\right)\right) \rightarrow d\right) \ell_{4} \\
& =\ell \varphi(d) .
\end{aligned}
$$

Hence, the above composite mapping is the canonical one from $H \otimes \operatorname{Hom}_{A^{\text {op }}}(D, A)$ to $\operatorname{Hom}_{A^{\text {op }}}(D, H \otimes A)$. It is hence bijective when $D$ is finitely presented in $\bmod \left(A^{\mathrm{op}}\right)$ or $\operatorname{dim}_{\mathbb{k}} H<\infty$. Accordingly, $\theta$ is bijective under the same assumption.

4.5. Tensor product of equivariant bimodules. Here, $A$ is a dg algebra.

4.5.1. The following result describes $\left(D \sharp^{\sigma} H\right) \underset{\Lambda}{\otimes}\left(D^{\prime} \sharp^{\tau} H\right)$ for equivariant $\operatorname{dg} A$ bimodules $D$ and $D^{\prime}$. Here is its setting

- $i, j \in \mathbb{Z}$ and $S$ is invertible as soon as $i<0$ or $j<0$,

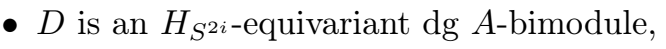

- $D^{\prime}$ is an $H_{S^{2 j}}$-equivariant dg $A$-bimodule,

- $\sigma, \tau$ are automorphisms of $H$ which satisfy the identities $\sigma(h)_{1} \otimes \sigma(h)_{2}=$ $S^{2 i}\left(h_{1}\right) \otimes \sigma\left(h_{2}\right)$ and $\tau(h)_{1} \otimes \tau(h)_{2}=S^{2 j}\left(h_{1}\right) \otimes \tau\left(h_{2}\right)$ in $H$, and such that $\sigma$ and $S^{2}$ commute, and such that $\tau$ and $S^{2}$ commute (for instance, $\sigma=$ $S^{+2 i} \circ \Xi_{\pi}^{r}$ and $\mu=S^{+2 j} \circ \Xi_{\pi^{\prime}}^{r}$ for algebra homomorphisms $\pi, \pi^{\prime}: H \rightarrow \mathbb{k}$ ).

Lemma. Keep the setting stated previously.

(1) There is a structure of $H_{S^{2(i+j)}}$-equivariant $d g$ A-bimodule on $D \otimes_{A} D^{\prime}$ such that $h \rightarrow\left(d \otimes d^{\prime}\right)=h_{1} \rightarrow d \otimes S^{2 i}\left(h_{2}\right) \rightarrow d^{\prime}$,

(2) $(D \sharp \sigma H) \otimes\left(D^{\prime} \sharp \tau\right) \simeq\left(D \otimes D^{\tau} D^{\prime}\right) \sharp \tau \circ \sigma H$ as $d g \Lambda$-bimodules. 
Proof. (1) The given action is indeed a structure of left $H$-module on $D \otimes D^{\prime}$ because

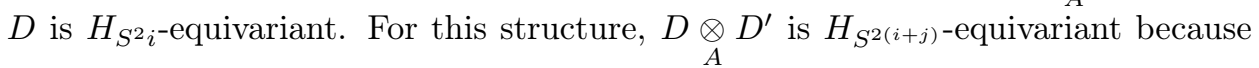

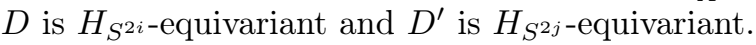

(2) Note the following identity in $H$

$$
\begin{aligned}
(\tau \circ \sigma)(h)_{1} \otimes(\tau \circ \sigma)(h)_{2} & =S^{2 j}\left(\sigma(h)_{1}\right) \otimes \tau\left(\sigma(h)_{2}\right) \\
& =S^{2 i+2 j}\left(h_{1}\right) \otimes \tau \circ \sigma\left(h_{2}\right) .
\end{aligned}
$$

Hence, $\left(D \underset{A}{\otimes} D^{\prime}\right) \sharp^{\tau \circ \sigma} H$ is well-defined.

Note the following identity in $\left(D \sharp^{\sigma} H\right) \underset{\Lambda}{\otimes}\left(D^{\prime} \sharp \tau\right)$ :

$$
(d \otimes \ell) \otimes_{\Lambda}\left(d^{\prime} \otimes \ell^{\prime}\right)=(d \otimes 1) \underset{\Lambda}{\otimes}\left(\ell_{1} \rightarrow d^{\prime} \otimes \tau\left(\ell_{2}\right) \ell^{\prime}\right) .
$$

Therefore, there is a well-defined linear mapping

$$
\begin{aligned}
& (D \sharp \sigma H) \underset{\Lambda}{\otimes}\left(D^{\prime} \sharp \tau\right) \rightarrow\left(D \underset{A}{\otimes} D^{\prime}\right) \otimes H \\
& (d \otimes \ell) \stackrel{\Lambda}{\Lambda}_{(}\left(d^{\prime} \otimes \ell^{\prime}\right) \quad \mapsto \quad\left(d \underset{A}{\otimes} \ell_{1} \rightarrow d^{\prime}\right) \otimes \tau\left(\ell_{2}\right) \ell^{\prime} .
\end{aligned}
$$

There is also a well-defined linear mapping

$$
\begin{aligned}
& \left(D \underset{A}{\otimes} D^{\prime}\right) \otimes H \quad \rightarrow \quad\left(D \sharp^{\sigma} H\right) \underset{\Lambda}{\otimes}\left(D^{\prime} \sharp \tau\right) \\
& \left(d \underset{A}{A} d^{\prime}\right) \otimes \ell^{\prime} \mapsto \quad(d \otimes 1) \underset{\Lambda}{\otimes}\left(d^{\prime} \otimes \ell^{\prime}\right)
\end{aligned}
$$

In view of 4.5.1.1, these two linear mappings are inverse to each other. In order to prove the lemma, it therefore suffices to check that the latter mapping is a morphism of $\operatorname{dg} \Lambda$-bimodules from $\left(D \underset{A}{\otimes} D^{\prime}\right) \sharp \tau \circ \sigma H$ to $\left(D \sharp^{\sigma} H\right) \underset{\Lambda}{\otimes}\left(D^{\prime} \sharp^{\tau} H\right)$. This follows from the computations below made in $(D \sharp \sigma H) \underset{\Lambda}{\otimes}\left(D^{\prime} \sharp^{\tau} H\right)$ :

$$
\begin{aligned}
a\left((d \otimes 1) \otimes{ }_{\Lambda}\left(d^{\prime} \otimes \ell^{\prime}\right)\right) b & =(a(d \otimes 1)) \otimes \\
& =(a d \otimes 1) \otimes{ }_{\Lambda}\left(d^{\prime}\left(\ell_{1}^{\prime} \rightarrow b\right) \otimes \ell_{2}^{\prime}\right)
\end{aligned}
$$

and

$$
\begin{aligned}
& h\left((d \otimes 1) \underset{\Lambda}{\otimes}\left(d^{\prime} \otimes \ell^{\prime}\right)\right) k=\quad=\quad(h(d \otimes 1)) \otimes_{\Lambda}\left(\left(d^{\prime} \otimes \ell^{\prime}\right) k\right) \\
& =\quad\left(h_{1} \rightarrow d \otimes \sigma\left(h_{2}\right)\right) \otimes\left(d^{\prime} \otimes \ell^{\prime} k\right)
\end{aligned}
$$

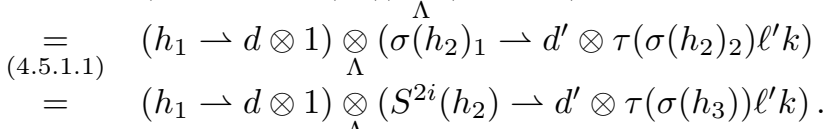

4.5.2. In this paragraph, $S$ need not be invertible. Let $D$ be an $H_{S^{2}}$-equivariant dg $A$-bimodule and let $\sigma \in \operatorname{Aut}_{\mathbb{k}-\operatorname{alg}}(H)$ satisfy the identity $\sigma(h)_{1} \otimes \sigma(h)_{2}=S^{2}\left(h_{1}\right) \otimes$ $\sigma\left(h_{2}\right)$ in $H$ and commute with $S^{2}$. The preceding result provides a description of $T_{A \sharp H}(D \sharp \sigma H)$. This is used later when discussing on Calabi-Yau completions.

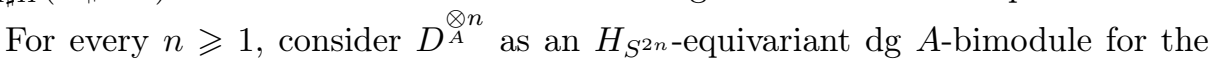
following action of $H$ (see 4.5.1

$(4.5 .2 .1) h \rightarrow\left(d_{1}{\underset{A}{A}}_{\otimes} \cdots{\underset{A}{\otimes}}_{n} d_{n}\right)=h_{1} \rightarrow d_{1}{\underset{A}{\otimes}} S^{2}\left(h_{2}\right) \rightarrow d_{2} \underset{A}{\otimes} \cdots \underset{A}{\otimes} S^{2(n-1)}\left(h_{n}\right) \rightarrow d_{n}$.

According to 4.5.1 there exists an isomorphism of algebras

$$
T_{A}(D) \otimes H \stackrel{\sim}{\longrightarrow} T_{A \sharp H}\left(D \sharp{ }^{\sigma} H\right)
$$


which extends the identity maps $A \rightarrow A$ and $H \rightarrow H$ as well as the mapping $D \rightarrow D \sharp{ }^{\sigma} H, d \mapsto d \otimes 1$, and where $T_{A}(D) \otimes H$ is endowed with the product such that $T_{A}(D)$ and $H$ are subalgebras in the natural way and

$$
h \times\left(d_{1} \otimes_{A} \cdots \otimes_{A} d_{n}\right)=h_{1} \rightarrow\left(d_{1} \otimes_{A} \cdots \otimes_{A} d_{n}\right) \otimes \sigma^{n}\left(h_{2}\right) .
$$

This dg algebra with underlying complex $T_{A}(D) \otimes H$ is denoted by $T_{A}(D) \sharp \sigma^{*} H$. If $\sigma=\operatorname{Id}_{H}$, then $T_{A}(D)$ is an $H$-module dg algebra and $T_{A}(D) \sharp \sigma^{*} H=T_{A}(D) \sharp H$.

4.6. On invertible $\Lambda$-bimodules. Assume that $A$ is a $\mathbb{k}$-algebra and that $S$ is invertible. The following results relate equivariant $A$-bimodules which are invertible as $A$-bimodules to invertible $\Lambda$-bimodules. Recall that an $A$-bimodule $D$ is called invertible if there exists an $A$-bimodule $D^{\prime}$ such that $D \underset{A}{\otimes} D^{\prime} \simeq D^{\prime} \otimes_{A} D \simeq A$ in $\bmod \left(A^{e}\right)$. In view of the adjunctions

$$
\begin{array}{cc}
\bmod (A) & \bmod \left(A^{\mathrm{op}}\right) \\
\underset{A}{\otimes}-\left(\uparrow_{A} \operatorname{Hom}_{A}(D,-)\right. & -\underset{A}{\otimes} D\left(\operatorname{Hom}_{A} \text { op }(D,-)\right. \\
\bmod (A) & \bmod \left(A^{\mathrm{op}}\right)
\end{array}
$$

the bimodule $D$ is invertible if and only if the canonical morphisms $\operatorname{Hom}_{A}(D, A) \otimes_{A}$ $D \rightarrow A$ and $D \otimes_{A} \operatorname{Hom}_{A^{\text {op }}}(D, A) \rightarrow A$ are bijective.

4.6.1. The following result gives a sufficient condition for $D \sharp{ }^{\sigma} H$ to be invertible.

Proposition. Let $H$ be a Hopf algebra with invertible antipode. Let $A$ be an $H$ module $\mathbb{k}$-algebra. Let $D$ be an $H_{S^{2 i}}$-equivariant $A$-bimodule (for some $i \in \mathbb{Z}$ ) which is invertible as an A-bimodule. Let $\sigma$ be an automorphism of $H$ which commutes with $S^{2}$ and satisfies the identity $\sigma(h)_{1} \otimes \sigma(h)_{2}=S^{2 i}\left(h_{1}\right) \otimes \sigma\left(h_{2}\right)$ in $H$. Then, $D \sharp{ }^{\sigma} H$ is an invertible $\Lambda$-bimodule.

Proof. The conclusion is a consequence of following the assertion proved below: $\operatorname{Hom}_{A}(D, A) \sharp^{\sigma^{-1}} H$ and $\operatorname{Hom}_{A^{\text {op }}}(D, A) \sharp^{\sigma^{-1}} H$ are right and left inverses of $D \sharp{ }^{\sigma} H$, respectively. Note that $\operatorname{Hom}_{A}(D, A)$ and $\operatorname{Hom}_{A^{\text {op }}}(D, A)$ are $H_{S^{-2 i}}$-equivariant $A$ bimodules (see 4.4.1) and $\sigma^{-1}$ satisfies the identity $\sigma^{-1}(h)_{1} \otimes \sigma^{-1}(h)_{2}=S^{-2 i}\left(h_{1}\right) \otimes$ $\sigma^{-1}\left(h_{2}\right)$.

Using part (2) of the lemma in 4.5.1 yields isomorphisms of $\Lambda$-bimodules

$$
\begin{aligned}
& \left(D \sharp^{\sigma} H\right) \underset{\Lambda}{\otimes}\left(\operatorname{Hom}_{A}(D, A) \sharp^{\sigma^{-1}} H\right) \simeq\left(D \underset{A}{\otimes} \operatorname{Hom}_{A}(D, A)\right) \sharp H \\
& \left(\operatorname{Hom}_{A^{\mathrm{op}}}(D, A) \sharp{ }^{\sigma^{-1}} H\right){\underset{\Lambda}{\otimes}}_{(D \sharp}\left(D \sharp^{\sigma} H\right) \simeq\left(\operatorname{Hom}_{A^{\mathrm{op}}}(D, A) \underset{A}{\otimes} D\right) \sharp H .
\end{aligned}
$$

Note that the $D \otimes \otimes_{A} \operatorname{Hom}_{A}(D, A)$ and $\operatorname{Hom}_{A}$ op $(D, A) \underset{A}{\otimes} D$ are $H$-equivariant $A$ bimodules (see 4.5 .1 . Moreover, the following mappings are isomorphisms in $\bmod \left(A^{e}\right)$

$$
\begin{aligned}
& D \underset{A}{\otimes} \operatorname{Hom}_{A}(D, A) \rightarrow A \quad \operatorname{Hom}_{A^{\mathrm{op}}}(D, A) \underset{A}{\otimes} D \rightarrow A \\
& d \otimes f \mapsto f(d) \quad f \otimes d \mapsto f(d) .
\end{aligned}
$$

These are actually $H$-linear as proved by the two following computations where $f$ lies in $\operatorname{Hom}_{A}(D, A)$ and $\operatorname{Hom}_{A}$ op $(D, A)$, respectively

$$
\begin{aligned}
\left(S^{2 i}\left(h_{2}\right) \rightarrow f\right)\left(h_{1} \rightarrow d\right) & =h_{3} \rightarrow f\left(S^{-1}\left(h_{2}\right) \rightarrow\left(h_{1} \rightarrow d\right)\right) \\
& =h \rightarrow f(d)
\end{aligned}
$$

and

$$
\begin{aligned}
\left(h_{1} \rightarrow f\right)\left(S^{-2 i}\left(h_{2}\right) \rightarrow d\right) & =h_{1} \rightarrow f\left(S^{1-2 i}\left(h_{2}\right) \rightarrow\left(S^{-2 i}\left(h_{3}\right) \rightarrow d\right)\right) \\
& =h \rightarrow f(d) .
\end{aligned}
$$


Therefore, $D \underset{A}{\otimes} \operatorname{Hom}_{A}(D, A) \simeq A$ and $\operatorname{Hom}_{A^{\mathrm{op}}}(D, A) \underset{A}{\otimes} D \simeq A$ as $H$-equivariant $A$-bimodules. Thus, there are isomorphisms of $\Lambda$-bimodules

$$
\begin{aligned}
& \left(D \otimes \operatorname{Hom}_{A}(D, A)\right) \sharp H \simeq \Lambda \\
& \left(\operatorname{Hom}_{A^{\text {op }}}(D, A) \underset{A}{\otimes} D\right) \sharp H \simeq \Lambda .
\end{aligned}
$$

This proves that $\operatorname{Hom}_{A}(D, A) \sharp^{\sigma^{-1}} H$ and $\operatorname{Hom}_{A^{\text {op }}}(D, A) \sharp^{\sigma^{-1}} H$ are right inverse and left inverse to $D \sharp{ }^{\sigma} H$, respectively.

4.6.2. The following result gives a necessary condition for $D \sharp^{\sigma} H$ to be invertible.

Proposition. Let $H$ be a Hopf algebra with invertible antipode. Let $A$ be an $H$ module $\mathbb{k}$-algebra. Let $D$ be an $H_{S^{2 i}}$-equivariant $A$-bimodule. Let $\sigma \in \operatorname{Aut}_{\mathbb{k}-\operatorname{alg}}(H)$ be such that $\sigma \circ S^{2}=S^{2} \circ \sigma$ and such that the identity $\sigma(h)_{1} \otimes \sigma(h)_{2}=S^{2 i}\left(h_{1}\right) \otimes \sigma\left(h_{2}\right)$ holds in $H$. Assume that $D \sharp^{\sigma} H$ is invertible as a $\Lambda$-bimodule. Then, $D$ is invertible as an A-bimodule.

Proof. Since $\left(D \sharp^{\sigma} H\right) \otimes A \simeq D$ in $\bmod (A)$, and since $D \sharp^{\sigma} H$ is invertible as a $\Lambda$ bimodule, then $D$ is finitely generated and projective in $\bmod (A)$. Similarly, since

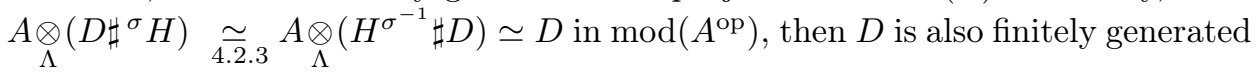
and projective in $\bmod \left(A^{\mathrm{op}}\right)$.

In order to prove that $D$ is invertible as an $A$-bimodule, it suffices to prove that the canonical mappings $D \otimes_{A} \operatorname{Hom}_{A}(D, A) \rightarrow A$ and $\operatorname{Hom}_{A^{\mathrm{op}}}(D, A) \otimes_{A} D \rightarrow A$ are bijective. Consider the former one. The following mapping is an isomorphism in $\bmod \left(\Lambda^{e}\right)(\operatorname{see} 4.4 .1$ and 4.5 .1$)$

$$
\begin{aligned}
\left(D \otimes \operatorname{Hom}_{A}(D, A)\right) \sharp H & \longrightarrow\left(D \sharp \sharp^{\sigma} H\right) \underset{\Lambda}{\otimes}\left(\operatorname{Hom}_{A}(D, A) \sharp{ }^{\sigma^{-1}} H\right) \\
(d \otimes \varphi) \otimes \ell & \longmapsto(d \otimes 1) \underset{\Lambda}{\otimes}(\varphi \otimes \ell)
\end{aligned}
$$

Combining the isomorphisms given in parts (1) and (2) of 4.4.2 yields the following isomorphism in $\bmod \left(\Lambda^{e}\right)$

$$
\begin{aligned}
\operatorname{Hom}_{A}(D, A) \sharp^{\sigma^{-1} H} & \longrightarrow \operatorname{Hom}_{\Lambda}\left(D \sharp^{\sigma} H, \Lambda\right) \\
\varphi \otimes \ell & \longmapsto\left(d \otimes h \mapsto \sigma^{-1}\left(h_{2}\right) \varphi\left(S^{-1-2 i}\left(h_{1}\right) \rightarrow d\right) \ell\right) .
\end{aligned}
$$

Now, since $D \sharp{ }^{\sigma} H$ is invertible, the following canonical mapping is an isomorphism in $\bmod \left(\Lambda^{e}\right)$

$$
\left(D \sharp^{\sigma} H\right) \underset{\Lambda}{\otimes} \operatorname{Hom}_{\Lambda}\left(D \sharp^{\sigma} H, \Lambda\right) \rightarrow \Lambda .
$$

Thus, combining 4.6.2.1, 4.6.2.2 and 4.6.2.3 yields an isomorphism in $\bmod \left(\Lambda^{e}\right)$

$$
\begin{aligned}
\left(D \otimes \operatorname{Hom}_{A}(D, A)\right) \sharp H & \rightarrow \Lambda \\
(d \otimes \varphi) \otimes \ell & \mapsto \varphi(d) \ell .
\end{aligned}
$$

Note that the canonical mapping $D \otimes \operatorname{Hom}_{A}(D, A) \rightarrow A$ is obtained upon applying $-\otimes A: \bmod \left(A^{e}\right) \rightarrow \bmod (A)$ to 4.6 .2 .4 , hence it is bijective.

Using similar considerations proves that the canonical mapping $\operatorname{Hom}_{A^{\text {op }}}(D, A) \otimes$ $D \rightarrow A$ is bijective. Thus, $A$ is invertible as an $A$-bimodule.

4.6.3. Combining the results in 4.6.1 and 4.6.2 yields the following corollary.

Corollary. Let $H$ be a Hopf algebra with invertible antipode. Let $A$ be an $H$ module $\mathbb{k}$-algebra. Let $D$ be an $H_{S^{2 i}}$-equivariant $A$-bimodule (for some $i \in \mathbb{Z}$ ). Let $\sigma$ be an automorphism of $H$ which commutes with $S^{2}$ and satisfies the identity $\sigma(h)_{1} \otimes \sigma(h)_{2}=S^{2 i}\left(h_{1}\right) \otimes \sigma\left(h_{2}\right)$ in $H$. Then, the following assertions are equivalent. 
(i) $D$ is invertible as an A-bimodule.

(ii) $D \sharp^{\sigma} H$ is invertible as an $A \sharp H$-bimodule.

\section{The inverse DuAlising COMPlex of $A \sharp H$}

This section assumes that $S$ is invertible and describes $\mathrm{RHom}_{\Lambda^{e}}\left(\Lambda, \Lambda^{e}\right)$. Starting from the observations that $A \in \mathcal{C}\left(\Delta_{0}\right)$ and $\Lambda^{e} \otimes_{\Delta_{0}} A \simeq \Lambda$ in $\mathcal{C}\left(\Lambda^{e}\right)$, the description is obtained by transforming $\operatorname{RHom}_{\Lambda^{e}}\left(\Lambda^{e} \otimes_{\Delta_{0}} A, \Lambda^{e}\right)$ using a series of adjunctions and the existence of some $D_{A} \in \mathcal{C}\left(\Delta_{1}\right)$ such that $D_{A} \simeq \operatorname{RHom}_{A^{e}}\left(A, A^{e}\right)$ in $\mathcal{D}\left(A^{e}\right)$. For this purpose, Section 5.1 collects some needed identities in $\Delta_{0}$ and $\Delta_{1}$, Section 5.2 proves technical details used in the series of adjunctions, Section 5.3 establishes a sufficient condition for $\Lambda$ to be homologically smooth, Section 5.4 introduces $D_{A}$, and Section 5.5 gives the description of $\operatorname{RHom}_{\Lambda^{e}}\left(\Lambda, \Lambda^{e}\right)$.

\subsection{Identities on the involved dg algebras.}

5.1.1. The following identities hold true in $\Delta_{0}$

$$
\begin{aligned}
h \times(a \otimes b) & =\left(\left(h_{1} \rightarrow a\right) \otimes\left(h_{3} \rightarrow b\right)\right) \times h_{2} \\
& =\left(\left(h_{1} \otimes S\left(h_{3}\right)\right) \rightarrow(a \otimes b)\right) \times h_{2} . \\
(a \otimes b) \times h= & h_{2} \times\left(\left(S^{-1}\left(h_{1}\right) \rightarrow a\right) \otimes\left(S\left(h_{3}\right) \rightarrow b\right)\right) \\
= & h_{2} \times\left(\left(S^{-1}\left(h_{1}\right) \otimes S^{2}\left(h_{3}\right)\right) \rightarrow(a \otimes b)\right) .
\end{aligned}
$$

The following identities hold true in $\Delta_{1}$

$$
\begin{gathered}
h \times(a \otimes b)=\left(h_{1} \rightarrow a \otimes S^{2}\left(h_{3}\right) \rightarrow b\right) \times h_{2} . \\
(a \otimes b) \times h=h_{2} \times\left(S^{-1}\left(h_{1}\right) \rightarrow a \otimes S^{3}\left(h_{3}\right) \rightarrow b\right) .
\end{gathered}
$$

5.1.2. Dg module structures over $A$ and $A^{e}$. There is a natural structure of left dg $\Lambda$-module on $A$ defined by $(a h) \rightarrow x=a(h \rightarrow x)$. Also there is a natural structure of left $\operatorname{dg} \Delta_{0}$-module on $A$ defined by $(a \otimes b \otimes h) \cdot x=(-1)^{\operatorname{deg}(b) \operatorname{deg}(x)} a(h \rightarrow x) b$.

Lemma. There is an isomorphism $\Lambda^{e} \otimes A \rightarrow \Lambda$ in $\mathcal{C}\left(\Lambda^{e}\right)$ which maps $(u \otimes v) \otimes a$ to $(-1)^{\operatorname{deg}(v) \operatorname{deg}(a)} u a v$ (where $u, v \in \Lambda$ and $a \in A, v$ and a being homogeneous).

Proof. Let $\mu: \Lambda^{e} \otimes A \rightarrow \Lambda$ be defined by $\mu((u \otimes v) \otimes a)=u a v$. Then

$$
\mu\left(\left((u \otimes v) \times\left(h_{1} \otimes S\left(h_{2}\right)\right)\right) \otimes a\right)= \pm u \underbrace{h_{1} a S\left(h_{2}\right)}_{h \rightarrow a} v=\mu((u \otimes v) \otimes(h \rightarrow a))
$$

and $\mu(((u \otimes v) \times(b \otimes c)) \otimes a)= \pm u b a c v= \pm \mu((u \otimes v) \otimes(b a c)$. Whence the existence of the morphism in $\mathcal{C}\left(\Lambda^{e}\right)$ given in the statement of the lemma. Denote it by $\nu$.

Let $\lambda: \Lambda \rightarrow \Lambda^{e} \otimes A$ be defined by $a h \mapsto(a \otimes h) \otimes 1$. Then $\lambda$ is a right inverse for $\mu$ and

$$
\begin{aligned}
\lambda \circ \mu((a h \otimes b k) \otimes c) & = \pm \lambda(a h c b k)= \pm\left(a\left(h_{1} \rightarrow c\right)\left(h_{2} \rightarrow b\right) \otimes h_{3} k\right) \otimes 1 \\
& = \pm\left(a \otimes h_{3} k\right) \otimes\left(h_{1} \rightarrow c\right)\left(h_{2} \rightarrow b\right) \\
& =\left(a \otimes\left(h_{2} \rightarrow b\right) h_{3} k\right) \otimes\left(h_{1} \rightarrow c\right) \\
& =\left(\left(a \otimes\left(h_{3} \rightarrow b\right) h_{4} k\right) \times\left(h_{1} \otimes S\left(h_{2}\right)\right)\right) \otimes_{\Delta_{0}} c \\
& =(a h \otimes b k) \otimes c .
\end{aligned}
$$

Thus, $\lambda$ is an isomorphism in $\mathcal{C}\left(\Lambda^{e}\right)$. 


\subsection{Useful (bi)module structures on morphism spaces.}

Lemma. Let $U \in \mathcal{C}\left(\Delta_{1}\right)$ and $Y \in \mathcal{C}\left(H^{\mathrm{op}}\right)$.

(1) $U \otimes H^{e}$ has a structure of $d g H-\Lambda^{e}$-bimodule where the action of $H$ is given by $\ell \rightarrow(u \otimes h \otimes k)=\ell_{2} \rightarrow u \otimes S^{2}\left(\ell_{3}\right) h \otimes k S\left(\ell_{1}\right)$ and the action of $\Lambda^{e}$ is given (in bimodule notation) by

$$
\begin{aligned}
a(u \otimes h \otimes k) b & =\left(\left(S^{-1}\left(k_{1}\right) \rightarrow a\right) u\left(h_{1} \rightarrow b\right)\right) \otimes h_{2} \otimes k_{2} \\
h^{\prime}(u \otimes h \otimes k) k^{\prime} & =u \otimes h k^{\prime} \otimes h^{\prime} k,
\end{aligned}
$$

(2) the canonical mapping $U \otimes H^{e} \rightarrow U \underset{A^{e}}{\otimes} \Lambda^{e}$ is both $H$-linear and $\Lambda^{e}$-linear,

(3) $U \otimes H \otimes Y$ has a structure of $d g$ right $\Lambda^{e}$-module such that (in bimodule notation) $a(u \otimes \ell \otimes y) b=a u\left(\ell_{1} \rightarrow b\right) \otimes \ell_{2} \otimes y$ and $h(u \otimes \ell \otimes y) k=\left(h_{1} \rightarrow\right.$ $u) \otimes S^{2}\left(h_{2}\right) \ell k \otimes y<S^{-1}\left(h_{3}\right)$,

(4) the mapping $U \otimes H \otimes Y \rightarrow Y \underset{H}{\otimes}\left(U \otimes H^{e}\right)$ defined by

$$
u \otimes \ell \otimes y \mapsto(-1)^{\operatorname{deg}(y) \operatorname{deg}(u)} y \otimes(u \otimes \ell \otimes 1)
$$

is a $\Lambda^{e}$-linear isomorphism.

Proof. (1) By construction the given actions define a structure of $\operatorname{dg} H-\left(H^{e}\right)$ bimodule. The given action of $A^{e}$ on $U \otimes H^{e}$ may be rewritten as the following composite map

$$
\left(U \otimes H^{e}\right) \otimes A^{e} \stackrel{\mathrm{Id} \otimes \tau}{\longrightarrow} U \otimes A^{e} \otimes H^{e} \stackrel{\alpha \otimes \mathrm{Id}}{\longrightarrow} U \otimes H^{e} .
$$

Here, $\tau: H^{e} \otimes A^{e} \rightarrow A^{e} \otimes H^{e}$ is defined by $\tau(h \otimes k \otimes a \otimes b)=\left(h_{1} \rightarrow a \otimes S^{-1}\left(k_{1}\right) \rightarrow\right.$ b) $\times\left(h_{2} \otimes k_{2}\right)$, and $\alpha: U \otimes A^{e} \rightarrow U$ is the action of $A^{e}$ on $U$ inherited from the action of $\Delta_{1}$. These considerations together with 2.2.2.1 explain that the given actions in the statement of the lemma define a structure of right $\operatorname{dg} \Lambda^{e}$-module on $U \otimes H^{e}$. Hence, there only remains to prove that the given actions of $H$ on the left and of $A^{e}$ on the right commute in order to prove that $U \otimes H^{e} \in \mathcal{C}\left(H \otimes\left(\Lambda^{e}\right)^{\mathrm{op}}\right)$. To this end, adopt the $A$-bimodule notation to show that $a(\ell \rightarrow(u \otimes h \otimes k)) b$ is equal to

$$
\begin{aligned}
& a\left(\ell_{2} \rightarrow u \otimes S^{2}\left(\ell_{3}\right) h \otimes k S\left(\ell_{1}\right)\right) b \\
= & \left(\left(\left(\ell_{2} S^{-1}\left(k_{1}\right)\right) \rightarrow a\right)\left(\ell_{3} \rightarrow u\right)\left(\left(S^{2}\left(\ell_{4}\right) h_{1}\right) \rightarrow b\right)\right) \otimes S^{2}\left(\ell_{5}\right) h_{2} \otimes k_{2} S\left(\ell_{1}\right) \\
= & \left(\ell_{2} \rightarrow\left(\left(S^{-1}\left(k_{1}\right) \rightarrow a\right) u\left(h_{1} \rightarrow b\right)\right)\right) \otimes S^{2}\left(\ell_{3}\right) h_{2} \otimes k_{2} S\left(\ell_{1}\right) \\
= & \ell \rightarrow(a(u \otimes h \otimes k) b) .
\end{aligned}
$$

(2) The action of $H$ on $U \otimes \Lambda^{e}$ which is being considered here is the one given in part (1) of the lemma in 4.3 . The given mapping is $H$-linear. Note that the structure of right $\operatorname{dg} \Lambda^{e}$-module of $U \underset{A^{e}}{\otimes} \Lambda^{e}$ is inherited from the one of $\Lambda^{e}$ itself. Hence, the given mapping is $\Lambda^{e}$-linear.

(3) The given actions define structures of right $\mathrm{dg} H^{e}$-module and right $\mathrm{dg} A^{e}$ module on $U \otimes H \otimes Y$. They form a structure of right $\operatorname{dg} \Lambda^{e}$-module in view of the following computations (with bimodule notation)

$$
\begin{aligned}
\left(h_{1} \rightarrow a\right)\left(h_{2}(u \otimes \ell \otimes y)\right)= & \left(h_{1} \rightarrow a\right)\left(h_{2} \rightarrow u\right) \otimes S^{2}\left(h_{3}\right) \ell \otimes y \leftarrow S^{-1}\left(h_{4}\right) \\
= & h(a(u \otimes h \otimes y)), \\
\left((u \otimes \ell \otimes y)\left(k_{1} \rightarrow b\right)\right) k_{2}= & u\left(\ell_{1} \rightarrow\left(k_{1} \rightarrow b\right)\right) \otimes \ell_{2} k_{2} \otimes y \\
& =((u \otimes \ell \otimes y) k) b \\
h((u \otimes \ell \otimes y) b)= & h_{1} \rightarrow\left(u\left(\ell_{1} \rightarrow b\right)\right) \otimes S^{2}\left(h_{2}\right) \ell_{2} \otimes y \leftarrow S^{-1}\left(h_{3}\right) \\
= & \left(h_{1} \rightarrow u\right)\left(\left(S^{2}\left(h_{2}\right) \ell_{1}\right) \rightarrow b\right) \otimes S^{2}\left(h_{3}\right) \ell_{2} \otimes y \leftarrow S^{-1}\left(h_{4}\right) \\
= & (h(u \otimes \ell \otimes y)) b .
\end{aligned}
$$


(4) Since $U \otimes H^{e} \in \mathcal{C}\left(H \otimes\left(\Lambda^{e}\right)^{\text {op }}\right)$, then $Y \underset{H}{\otimes}\left(U \otimes H^{e}\right) \in \mathcal{C}\left(\left(\Lambda^{e}\right)^{\text {op }}\right)$. Note the following identity in $U \otimes H^{e}$

$$
(u \otimes h \otimes k)=S^{-1}\left(k_{3}\right) \rightarrow\left(k_{1} \rightarrow u \otimes S^{2}\left(k_{2}\right) h \otimes 1\right),
$$

Indeed,

$$
\begin{aligned}
S^{-1}\left(k_{3}\right) \rightarrow\left(k_{1} \rightarrow u \otimes S^{2}\left(k_{2}\right) h \otimes 1\right) & =S^{-1}\left(k_{2}\right) \rightarrow\left(k_{1} \rightarrow u\right) \otimes h \otimes k_{3} \\
& =(u \otimes h \otimes k) .
\end{aligned}
$$

The following computations in $Y \underset{H}{\otimes}\left(U \otimes H^{e}\right)$ show that the mapping from $U \otimes H \otimes Y$ to $Y \underset{H}{\otimes}\left(U \otimes H^{e}\right)$ given in the statement of the lemma is $\Lambda^{e}$-linear,

$$
\begin{aligned}
a(y \underset{H}{\otimes}(u \otimes \ell \otimes 1)) b & =(-1)^{\operatorname{def}(y) \operatorname{deg}(a)} y \underset{H}{\otimes}(a(u \otimes \ell \otimes 1) b) \\
& =(-1)^{\operatorname{def}(y) \operatorname{deg}(a)} \underset{H}{\underset{H}{\otimes}}\left(a u\left(\ell_{1} \rightarrow b\right) \otimes \ell_{2} \otimes 1\right)
\end{aligned}
$$

and

$$
\begin{array}{rll}
h(y \underset{H}{\otimes}(u \otimes \ell \otimes 1)) k & = & y \underset{H}{\otimes} h(u \otimes \ell \otimes 1) k \\
& = & y \otimes_{H}^{\otimes}(u \otimes \ell k \otimes h) \\
= & y \underset{H}{\otimes} S^{-1}\left(h_{3}\right) \rightarrow\left(h_{1} \rightarrow u \otimes S^{2}\left(h_{2}\right) \ell k \otimes 1\right) \\
\stackrel{5.2 .0 .1}{=} & y \leftarrow S^{-1}\left(h_{3}\right) \underset{H}{\otimes}\left(h_{1} \rightarrow u \otimes S^{2}\left(h_{2}\right) \ell k \otimes 1\right) .
\end{array}
$$

The given mapping is bijective with inverse $Y \underset{H}{\otimes}\left(U \otimes H^{e}\right) \rightarrow U \otimes H \otimes Y$ welldefined by $y \otimes(u \otimes h \otimes k) \mapsto(-1)^{\operatorname{deg}(y) \operatorname{deg}(u)}\left(k_{1} \rightarrow u\right) \otimes S^{2}\left(k_{2}\right) h \otimes y<S^{-1}\left(k_{3}\right)$ (see 5.2 .0 .1$)$.

5.3. Preliminaries on homological algebra. This section gives some needed material to transform $\mathrm{RHom}_{\Lambda^{e}}\left(\Lambda, \Lambda^{e}\right)$ using adjunctions. It also proves that $\Lambda$ is homologically smooth if $A$ and $H$ are so.

5.3.1. The following result gives sufficient conditions for $\operatorname{Hom}_{A^{e}}(X, M)$ to be homotopically injective in $\mathcal{C}(H)$ given $M \in \mathcal{C}\left(\Lambda^{e}\right)$ and $X \in \mathcal{C}\left(\Delta_{0}\right)$. Note that the structure of left dg $H$-module on $\operatorname{Hom}_{A^{e}}(X, M)$ is taken from 4.3 (part (2)).

Lemma. Let $X \in \mathcal{C}\left(\Delta_{0}\right)$ and $M \in \mathcal{C}\left(\Lambda^{e}\right)$. For every $N \in \mathcal{C}(H)$, there is a natural structure of left dg $\Delta_{0}$-module on $\operatorname{Hom}_{\mathrm{k}}(N, M)$ such that (functorially)

$$
\operatorname{Hom}_{H}\left(N, \operatorname{Hom}_{A^{e}}(X, M)\right) \simeq \operatorname{Hom}_{\Delta_{0}}\left(X, \operatorname{Hom}_{\mathrm{k}}(N, M)\right) .
$$

As a consequence, $\operatorname{Hom}_{A^{e}}(X, M)$ is homotopically injective in $\mathcal{C}(H)$ if and only if $X$ is homotopically projective in $\mathcal{C}\left(\Delta_{0}\right)$.

Proof. On the one hand, the following action of $H$ on $\operatorname{Hom}_{\mathrm{k}}(N, M)$ is a structure of left dg $H$-module

$$
(h \rightarrow f)(n)=h_{1} f\left(S^{-1}\left(h_{2}\right) \rightarrow n\right) S\left(h_{3}\right) .
$$

On the other hand, the action of $A^{e}$ on $M$ defines a structure of left $\operatorname{dg} A^{e}$-module on $\operatorname{Hom}_{\mathrm{k}}(N, M)$. Those two structures form a structure of left $\operatorname{dg} \Delta_{0}$-module. This claim follows from the following computation where $f \in \operatorname{Hom}_{\mathrm{k}}(N, M)$,

$$
\begin{aligned}
(h \rightarrow(a f b))(n) & =h_{1} a f\left(S^{-1}\left(h_{2}\right) \rightarrow n\right) b S\left(h_{3}\right) \\
& =\left(h_{1} \rightarrow a\right) h_{2} f\left(S^{-1}\left(h_{3}\right) \rightarrow n\right) S\left(h_{4}\right)\left(h_{5} \rightarrow b\right) \\
& =\left(\left(h_{1} \rightarrow a\right)\left(h_{2} \rightarrow f\right)\left(h_{3} \rightarrow b\right)\right)(n) .
\end{aligned}
$$

Here is a mapping $F: \operatorname{Hom}_{H}\left(N, \operatorname{Hom}_{A^{e}}(X, M)\right) \rightarrow \operatorname{Hom}_{\Delta_{0}}\left(X, \operatorname{Hom}_{\mathrm{k}}(N, M)\right)$ which fits the conclusion of the lemma. Let $\lambda \in \operatorname{Hom}_{H}\left(N, \operatorname{Hom}_{A^{e}}(X, M)\right)$ and 
define $\mu \in \operatorname{Hom}_{\mathrm{k}}\left(X, \operatorname{Hom}_{\mathbb{k}}(N, M)\right)$ by $\mu(x)(n)=(-1)^{\operatorname{deg}(n) \cdot \operatorname{deg}(x)} \lambda(n)(x)$. Then, $\mu$ is $\Delta_{0}$-linear. Indeed,

$$
\begin{aligned}
\mu((a \otimes b) x)(n) & =(-1)^{\operatorname{deg}(n) \cdot(\operatorname{deg}(a \otimes b)+\operatorname{deg}(x))} \lambda(n)((a \otimes b) x) \\
& =(-1)^{\operatorname{deg}(n) \cdot(\operatorname{deg}(a \otimes b)+\operatorname{deg}(x))+\operatorname{deg}(a \otimes b) \cdot \operatorname{deg}(\lambda(n))}(a \otimes b) \lambda(n)(x) \\
& =(-1)^{\operatorname{deg}(a \otimes b) \cdot \operatorname{deg}(\lambda)} \mu(x)(n)
\end{aligned}
$$

and

$$
\begin{array}{ccl}
(h \rightarrow \mu(x))(n) & \underset{15.1 .1}{=} & h_{1} \mu(x)\left(S^{-1}\left(h_{2}\right) \rightarrow n\right) S\left(h_{3}\right) \\
& = & \pm h_{1} \lambda\left(S^{-1}\left(h_{2}\right) \rightarrow n\right)(x) S\left(h_{3}\right) \\
= & \pm h_{1}\left(S^{-1}\left(h_{2}\right) \rightarrow \lambda(n)\right)(x) S\left(h_{3}\right) \\
& = & \pm h_{1} S\left(h_{2}\right) \lambda(n)\left(h_{3} \rightarrow x\right) h_{4} S\left(h_{5}\right) \\
& = & \mu(h \rightarrow x)(n),
\end{array}
$$

where \pm is the sign $(-1)^{\operatorname{deg}(x) \cdot \operatorname{deg}(n)}$. Thus, defining $F(\lambda)$ by $F(\lambda)=\mu$ yields an injective mapping

$$
F: \operatorname{Hom}_{H}\left(N, \operatorname{Hom}_{A^{e}}(X, M)\right) \rightarrow \operatorname{Hom}_{\Delta_{0}}\left(X, \operatorname{Hom}_{k}(N, M)\right) .
$$

Here is why $F$ is surjective. Let $\mu \in \operatorname{Hom}_{\Delta_{0}}\left(X, \operatorname{Hom}_{\mathrm{k}}(N, M)\right)$. For every $n$, define $\lambda(n) \in \operatorname{Hom}_{\mathbb{k}}(X, M)$ by $\lambda(n)(x)=(-1)^{\operatorname{deg}(x) \cdot \operatorname{deg}(n)} \mu(x)(n)$. Then, $\lambda(n)$ is $A^{e}$-linear because $\mu$ is $\Delta_{0}$-linear. Moreover, the mapping $N \rightarrow \operatorname{Hom}_{A^{e}}(X, M)$ defined by $n \mapsto \lambda(n)$ is $H$-linear:

$$
\begin{array}{ccl}
(h \rightarrow \lambda(n))(x) & = & S^{2}\left(h_{3}\right) \lambda(n)\left(S\left(h_{2}\right) \rightarrow x\right) S\left(h_{1}\right) \\
& = & \pm S^{2}\left(h_{3}\right) \mu\left(S\left(h_{2}\right) \rightarrow x\right)(n) S\left(h_{1}\right) \\
= & \pm S^{2}\left(h_{3}\right)\left(S\left(h_{2}\right) \rightarrow \mu(x)\right)(n) S\left(h_{1}\right) \\
= & \pm S^{2}\left(h_{5}\right) S\left(h_{4}\right) \mu(x)\left(h_{3} \rightarrow n\right) S^{2}\left(h_{2}\right) S\left(h_{1}\right) \\
& = & \\
& = & \lambda(h \rightarrow n)(x),
\end{array}
$$

where \pm is the degree $(-1)^{\operatorname{deg}(x) \cdot \operatorname{deg}(n)}$. Thus, $\lambda \in \operatorname{Hom}_{H}\left(N, \operatorname{Hom}_{A^{e}}(X, M)\right)$ and $F(\lambda)=\mu$. This proves that $F$ is surjective, and hence bijective. And it is functorial. Whence the first assertion of the lemma. The remaining assertion follows immediately.

5.3.2. The following result asserts that $\Lambda$ is homologically smooth if $A$ and $H$ are so. For ordinary algebras this was already proved in [26, Proposition 2.11].

Proposition. Let $H$ be a Hopf algebra with invertible antipode. Let $A$ be an $H$ module dg algebra. If ${ }_{H} \mathbb{k} \in \operatorname{per}(H)$ and $A \in \operatorname{per}\left(A^{e}\right)$, then $\Lambda \in \operatorname{per}\left(\Lambda^{e}\right)$.

Proof. Let $P \rightarrow A$ be a cofibrant replacement in $\mathcal{C}\left(\Delta_{0}\right)$. This is also a cofibrant replacement in $\mathcal{C}\left(A^{e}\right)$ because $\Delta_{0} \simeq A^{e} \otimes H$ in $\mathcal{C}\left(A^{e}\right)$. Moreover, the induced morphism $\Lambda^{e} \underset{\Delta_{0}}{\otimes} P \rightarrow \Lambda^{e} \underset{\Delta_{0}}{\otimes} A \simeq \Lambda$ is a cofibrant replacement in $\mathcal{C}\left(\Lambda^{e}\right)$. Let $Q \rightarrow{ }_{H} \mathbb{k}$ be a cofibrant replacement in $\mathcal{C}(H)$. In order to prove the statement of the lemma, it suffices to prove that $\Lambda$ is compact in $\mathcal{D}\left(\Lambda^{e}\right)$, that is, for any given family $\left(M_{i}\right)_{i \in I}$ in $\mathcal{C}\left(\Lambda^{e}\right)$ with direct sum denoted by $M$, the canonical mapping $\oplus_{i \in I} \operatorname{Hom}_{\Lambda^{e}}\left(\Lambda^{e} \otimes\right.$ $\left.P, M_{i}\right) \rightarrow \operatorname{Hom}_{\Lambda^{e}}\left(\Lambda^{e} \underset{\Delta_{0}}{\otimes} P, M\right)$ is a quasi-isomorphism. By adjunction, this reduces to proving that the canonical mapping $\oplus_{i \in I} \operatorname{Hom}_{\Delta_{0}}\left(P, M_{i}\right) \rightarrow \operatorname{Hom}_{\Delta_{0}}(P, M)$ is a quasi-isomorphism.

Note that, if $N$ is either $M$ or one of the $M_{i}$, then $\operatorname{Hom}_{A^{e}}(P, N)$ has a structure of left dg $H$-module which is functorial in $N$ (see 4.3, part (2)) and such that there is a functorial isomorphism $\operatorname{Hom}_{\Delta_{0}}(P, N) \stackrel{\simeq}{\rightarrow} \operatorname{Hom}_{H}\left(H \mathbb{k}, \operatorname{Hom}_{A^{e}}(P, N)\right)$. 
Since $\operatorname{Hom}_{A^{e}}(P, M)$ is homotopically injective in $\mathcal{C}(H)$ (see 5.3.1), the functorial isomorphism $\operatorname{Hom}_{\Delta_{0}}(P, M) \stackrel{\simeq}{\rightarrow} \operatorname{Hom}_{H}\left(H \mathbb{k}, \operatorname{Hom}_{A^{e}}(P, M)\right)$ induces a quasiisomorphism

$$
\operatorname{Hom}_{\Delta_{0}}(P, M) \stackrel{\lambda}{\rightarrow} \operatorname{Hom}_{H}\left(Q, \operatorname{Hom}_{A^{e}}(P, M)\right) .
$$

Since $P \rightarrow A$ is a cofibrant replacement in $\mathcal{C}\left(A^{e}\right)$ and since $A \in \operatorname{per}\left(A^{e}\right)$, the canonical mapping $\oplus_{i \in I} \operatorname{Hom}_{A^{e}}\left(P, M_{i}\right) \rightarrow \operatorname{Hom}_{A^{e}}(P, M)$ is a quasi-isomorphism. Therefore it induces a quasi-isomorphism

$$
\operatorname{Hom}_{H}\left(Q, \oplus_{i \in I} \operatorname{Hom}_{A^{e}}\left(P, M_{i}\right)\right) \stackrel{\mu_{1}}{\longrightarrow} \operatorname{Hom}_{H}\left(Q, \operatorname{Hom}_{A^{e}}(P, M)\right) \text {. }
$$

Now, using that $Q \rightarrow{ }_{H} \mathbb{k}$ is a cofibrant replacement in $\mathcal{C}(H)$ and that ${ }_{H} \mathbb{k} \in \operatorname{per}(H)$, it follows that the canonical mapping below is a quasi-isomorphism

$$
\oplus_{i \in I} \operatorname{Hom}_{H}\left(Q, \operatorname{Hom}_{A^{e}}\left(P, M_{i}\right)\right) \stackrel{\mu_{2}}{\longrightarrow} \operatorname{Hom}_{H}\left(Q, \oplus_{i \in I} \operatorname{Hom}_{A^{e}}\left(P, M_{i}\right)\right) \text {. }
$$

The analogues of $\lambda$ for the $M_{i}$ (instead of for $M$ ) give rise to a quasi-isomorphism

$$
\oplus_{i \in I} \operatorname{Hom}_{\Delta_{0}}\left(P, M_{i}\right) \stackrel{\nu}{\rightarrow} \oplus_{i \in I} \operatorname{Hom}_{H}\left(Q, \operatorname{Hom}_{A^{e}}\left(P, M_{i}\right)\right) .
$$

Therefore, the canonical mapping $\oplus_{i \in I} \operatorname{Hom}_{\Delta_{0}}\left(P, M_{i}\right) \rightarrow \operatorname{Hom}_{\Delta_{0}}(P, M)$ is a quasiisomorphism because it fits into a commutative diagram

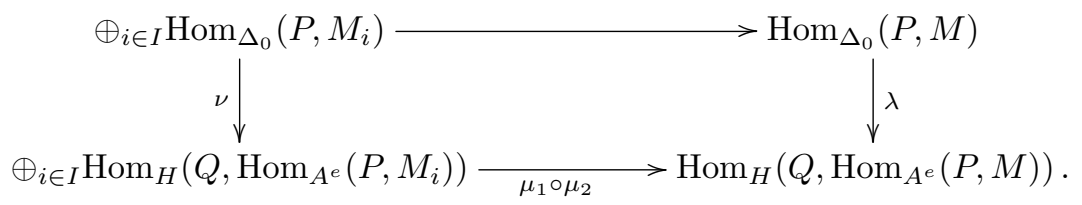

Thus, $\Lambda$ is compact in $\mathcal{D}\left(\Lambda^{e}\right)$, and hence $\Lambda \in \operatorname{per}\left(\Lambda^{e}\right)$.

5.4. Additional structure on the inverse dualising complex of $A$. The following is a direct consequence of 4.3 . Recall that $A^{e}$ is a $\mathrm{dg} H^{e}$-module by means of 2.2.2.3 and that, if $X \in \mathcal{C}\left(\Delta_{0}\right)$, then $\operatorname{Hom}_{A^{e}}\left(X, A^{e}\right)$ is a left $\operatorname{dg} \Delta_{1}$-module obtained by combining its natural structure of $\operatorname{dg} A$-bimodule and the structure of left dg $H$-module defined by 4.3.0.1).

Proposition. Let $H$ be a Hopf algebra with invertible antipode. Let $A$ be an $H$ module dg algebra. There exists $D_{A} \in \mathcal{C}\left(\Delta_{1}\right)$ such that $D_{A} \simeq \operatorname{RHom}_{A^{e}}\left(A, A^{e}\right)$ in $\mathcal{D}\left(\left(A^{e}\right)^{\mathrm{op}}\right)$. More precisely, if $P \rightarrow A$ is a quasi-isomorphism in $\mathcal{C}\left(\Delta_{0}\right)$ such that $P$ is cofibrant in $\mathcal{C}\left(A^{e}\right)$, then $D_{A}$ may be taken equal to $\operatorname{Hom}_{A^{e}}\left(P, A^{e}\right)$.

Whenever a cochain $\varphi$ of $\operatorname{Hom}_{A^{e}}\left(P, A^{e}\right)$ is given and denoted symbolically by $p \mapsto \varphi^{\prime}(p) \otimes \varphi^{\prime \prime}(p)$, the structure of $\operatorname{dg} \Delta_{1}$-module of $\operatorname{Hom}_{A^{e}}\left(P, A^{e}\right)$ is such that $h \rightarrow \varphi$ is the cochain

$$
p \mapsto S^{2}\left(h_{3}\right) \rightarrow \varphi^{\prime}\left(S\left(h_{2}\right) \rightarrow p\right) \otimes h_{1} \rightarrow \varphi^{\prime \prime}\left(S\left(h_{2}\right) \rightarrow p\right) .
$$

Note that, when $S^{2}=\operatorname{Id}_{H}$, then $(h \rightarrow \varphi)(p)=h_{3} \rightarrow \varphi^{\prime}\left(S\left(h_{2}\right) \rightarrow p\right) \otimes h_{1} \rightarrow$ $\varphi^{\prime \prime}\left(S\left(h_{2}\right) \rightarrow p\right)$.

Example. Consider the setting of the running example in 4.1. Let $P$ be the Koszul resolution $K^{\bullet}$. Given $X \in \mathfrak{g}$ and given a cochain $\varphi \in \operatorname{Hom}_{A^{e}}\left(K^{\bullet}, A^{e}\right)$, then $X \rightarrow \varphi$ is the cochain denoted by $\partial_{X}(\varphi)$

$$
\begin{aligned}
\partial_{X}(\varphi): K^{\bullet} & \rightarrow A^{e} \\
\omega & \mapsto \partial_{X}(\varphi(\omega))-\varphi\left(\partial_{X}(\omega)\right) .
\end{aligned}
$$

\subsection{An expression of an inverse dualising complex of $\Lambda$.}


5.5.1. Here is a description of $\operatorname{RHom}_{\Lambda^{e}}\left(\Lambda, \Lambda^{e}\right)$.

Proposition. Let $A$ be an $H$-module dg algebra where $H$ is a Hopf algebra with invertible antipode. Assume that both $A$ and $H$ are homologically smooth. Then, $\Lambda$ is homologically smooth and $D_{A} \otimes H \otimes E_{H} \simeq \operatorname{RHom}_{\Lambda^{e}}\left(\Lambda, \Lambda^{e}\right)$ in $\mathcal{D}\left(\left(\Lambda^{e}\right)^{\mathrm{op}}\right)$ where

- $D_{A}$ is any dg $\Delta_{1}$-module isomorphic to $\operatorname{RHom}_{A^{e}}\left(A, A^{e}\right)$ in $\mathcal{D}\left(A^{e}\right)$, see 5.4 .

- $E_{H} \in \mathcal{C}\left(H^{\mathrm{op}}\right)$ is any cofibrant replacement of $\mathrm{RHom}_{H}\left({ }_{H} \mathbb{k}, H\right)$,

- the right $d g \Lambda^{e}$-module structure is given (with bimodule notation) by

$$
\begin{aligned}
a(d \otimes \ell \otimes e) b & =a d\left(\ell_{1} \rightarrow b\right) \otimes \ell_{2} \otimes e \\
h(d \otimes \ell \otimes e) k & =h_{1} \rightarrow d \otimes S^{2}\left(h_{2}\right) \ell k \otimes e \leftarrow S^{-1}\left(h_{3}\right) .
\end{aligned}
$$

Proof. Let $P \rightarrow A$ be a quasi-isomorphism in $\mathcal{C}\left(\Delta_{0}\right)$ such that $P$ is cofibrant in $\mathcal{C}\left(A^{e}\right)$. Note that any cofibrant replacement of $A$ in $\mathcal{C}\left(\Delta_{0}\right)$ fits this requirement because $\Delta_{0} \simeq A^{e} \otimes H$ in $\mathcal{C}\left(A^{e}\right)$. Let $Q \rightarrow{ }_{H} \mathbb{k}$ be a cofibrant replacement in $\mathcal{C}(H)$. Then, $\operatorname{Hom}_{A^{e}}\left(P, A^{e}\right)$ may be used for $D_{A}$ (see 5.4) and $\operatorname{Hom}_{H}(Q, H)$ may be used for $E_{H}$. Let $\Lambda^{e} \rightarrow I$ be a fibrant replacement in $\mathcal{C}\left(\left(\Lambda^{e}\right)^{e}\right)$. Then, $\operatorname{Hom}_{\Lambda^{e}}(\Lambda, I)$ has a structure of right $\operatorname{dg} \Lambda^{e}$-module inherited by the one of $I$ and

$$
\operatorname{Hom}_{\Lambda^{e}}(\Lambda, I) \simeq \operatorname{RHom}_{\Lambda^{e}}\left(\Lambda, \Lambda^{e}\right) \text { in } \mathcal{D}\left(\left(\Lambda^{e}\right)^{\mathrm{op}}\right) .
$$

Since $\Lambda \simeq \Lambda^{e} \otimes_{\Delta_{0}} A$ in $\mathcal{C}\left(\Lambda^{e}\right)$ (see 5.1.2), there is an isomorphism in $\mathcal{C}\left(\left(\Lambda^{e}\right)^{\mathrm{op}}\right)$

$$
\operatorname{Hom}_{\Lambda^{e}}(\Lambda, I) \simeq \operatorname{Hom}_{\Delta_{0}}(A, I) .
$$

Since $\Lambda^{e} \simeq \Delta_{0} \otimes H$ in $\mathcal{C}\left(\Delta_{0}\right)$, then $I$ is fibrant in $\mathcal{C}\left(\Delta_{0}\right)$. Hence, the quasiisomorphism $P \rightarrow A$ induces a quasi-isomorphism in $\mathcal{C}\left(\left(\Lambda^{e}\right)^{\text {op }}\right)$

$$
\operatorname{Hom}_{\Delta_{0}}(A, I) \stackrel{\text { qis }}{\longrightarrow} \operatorname{Hom}_{\Delta_{0}}(P, I) \text {. }
$$

Using the functorial construction in 4.3 (part (2)), the structure of right $\operatorname{dg} \Lambda^{e_{-}}$ module on $\operatorname{Hom}_{A^{e}}(P, I)$ may be extended to a structure of dg $H-\Lambda^{e}$-bimodule such that there is an isomorphism in $\mathcal{C}\left(\left(\Lambda^{e}\right)^{\text {op }}\right)$

$$
\operatorname{Hom}_{\Delta_{0}}(P, I) \simeq \operatorname{Hom}_{H}\left({ }_{H} \mathbb{k}, \operatorname{Hom}_{A^{e}}(P, I)\right) .
$$

It follows from 5.3.1 that $\operatorname{Hom}_{A^{e}}(P, I)$ is homotopically injective in $\mathcal{C}(H)$. Therefore, $Q \rightarrow{ }_{H} \mathbb{k}$ induces a quasi-isomorphism in $\mathcal{C}\left(\left(\Lambda^{e}\right)^{\text {op }}\right)$

$$
\operatorname{Hom}_{H}\left({ }_{H} \mathbb{k}, \operatorname{Hom}_{A^{e}}(P, I)\right) \stackrel{\text { qis }}{\longrightarrow} \operatorname{Hom}_{H}\left(Q, \operatorname{Hom}_{A^{e}}(P, I)\right) \text {. }
$$

Like for $\operatorname{Hom}_{A^{e}}(P, I)$, the structure of right $\operatorname{dg} \Lambda^{e}$-module of $\operatorname{Hom}_{A^{e}}\left(P, \Lambda^{e}\right)$ extends to a structure of dg $H-\Lambda^{e}$-bimodule. Since $P$ and $Q$ are cofibrant in $\mathcal{C}\left(A^{e}\right)$ and $\mathcal{C}(H)$, respectively, the quasi-isomorphism $\Lambda^{e} \rightarrow I$ induces a quasi-isomorphism in $\mathcal{C}\left(\left(\Lambda^{e}\right)^{\text {op }}\right)$

$$
\operatorname{Hom}_{H}\left(Q, \operatorname{Hom}_{A^{e}}\left(P, \Lambda^{e}\right)\right) \stackrel{\text { qis }}{\longrightarrow} \operatorname{Hom}_{H}\left(Q, \operatorname{Hom}_{A^{e}}(P, I)\right) .
$$

Thanks to 4.3 (parts (1) and (6)), there is a structure of left dg $H$-module on $\operatorname{Hom}_{A^{e}}\left(P, A^{e}\right) \underset{A^{e}}{\otimes} \Lambda^{e}$ such that the canonical mapping from $\operatorname{Hom}_{A^{e}}\left(P, A^{e}\right) \underset{A^{e}}{\otimes} \Lambda^{e}$ to $\operatorname{Hom}_{A^{e}}\left(P, \Lambda^{e}\right)$ is $H$-linear. This is a quasi-isomorphism because $A \in \operatorname{per}\left(A^{e}\right)$. And it is $\Lambda^{e}$-linear (to the right) by functoriality of the involved constructions. It therefore induces a quasi-isomorphism in $\mathcal{C}\left(\left(\Lambda^{e}\right)^{\text {op }}\right)$

$$
\operatorname{Hom}_{H}\left(Q, \operatorname{Hom}_{A^{e}}\left(P, A^{e}\right) \underset{A^{e}}{\otimes} \Lambda^{e}\right) \stackrel{\text { qis }}{\longrightarrow} \operatorname{Hom}_{H}\left(Q, \operatorname{Hom}_{A^{e}}\left(P, \Lambda^{e}\right)\right) .
$$

Recall that $\operatorname{Hom}_{A^{e}}\left(P, A^{e}\right) \in \mathcal{C}\left(\Delta_{1}\right)$ as detailed in 4.3 (part (5)). Accordingly (see 5.2 parts $(1)$ and $(2)), \operatorname{Hom}_{A^{e}}\left(P, A^{e}\right) \otimes H^{e} \in \mathcal{C}\left(H \otimes\left(\Lambda^{e}\right)^{\mathrm{op}}\right)$ in such a way that the canonical mapping $\operatorname{Hom}_{A^{e}}\left(P, A^{e}\right) \otimes H^{e} \rightarrow \operatorname{Hom}_{A^{e}}\left(P, A^{e}\right) \underset{A^{e}}{\otimes} \Lambda^{e}$ is both $H$-linear 
and $\Lambda^{e}$-linear. It is an isomorphism because $\Lambda^{e} \simeq A^{e} \otimes H^{e}$ in $\mathcal{C}\left(A^{e}\right)$. Therefore, it induces an isomorphism in $\mathcal{C}\left(\left(\Lambda^{e}\right)^{\mathrm{op}}\right)$

$$
\operatorname{Hom}_{H}\left(Q, \operatorname{Hom}_{A^{e}}\left(P, A^{e}\right) \otimes H^{e}\right) \stackrel{\sim}{\longrightarrow} \operatorname{Hom}_{H}\left(Q, \operatorname{Hom}_{A^{e}}\left(P, A^{e}\right) \underset{A^{e}}{\otimes} \Lambda^{e}\right) .
$$

Since ${ }_{H} \mathbb{k} \in \operatorname{per}(H)$, the following canonical mapping is a quasi-isomorphism in $\mathcal{C}\left(\left(\Lambda^{e}\right)^{\text {op }}\right)$

$$
\operatorname{Hom}_{H}(Q, H) \underset{H}{\otimes}\left(\operatorname{Hom}_{A^{e}}\left(P, A^{e}\right) \otimes H^{e}\right) \stackrel{\text { qis }}{\longrightarrow} \operatorname{Hom}_{H}\left(Q, \operatorname{Hom}_{A^{e}}\left(P, A^{e}\right) \otimes H^{e}\right) .
$$

Finally, using the structure of left $\operatorname{dg} \Delta_{1}$-module on $\operatorname{Hom}_{A^{e}}\left(P, A^{e}\right)$, there is an associated structure of right $\operatorname{dg} \Lambda^{e}$-module on $\operatorname{Hom}_{A^{e}}\left(P, A^{e}\right) \otimes H \otimes \operatorname{Hom}_{H}(Q, H)$ introduced in 5.2 (part $(3))$. For this structure, there is an isomorphism in $\mathcal{C}\left(\left(\Lambda^{e}\right)^{\mathrm{op}}\right)$ (see 5.2 part (4))

$$
\operatorname{Hom}_{A^{e}}\left(P, A^{e}\right) \otimes H \otimes \operatorname{Hom}_{H}(Q, H) \stackrel{\sim}{\longrightarrow} \operatorname{Hom}_{H}(Q, H) \underset{H}{\otimes}\left(\operatorname{Hom}_{A^{e}}\left(P, A^{e}\right) \otimes H^{e}\right) .
$$

Therefore $\operatorname{RHom}_{\Lambda^{e}}\left(\Lambda, \Lambda^{e}\right) \simeq \operatorname{Hom}_{A^{e}}\left(P, A^{e}\right) \otimes H \otimes \operatorname{Hom}_{H}(Q, H)$ in $\mathcal{D}\left(\left(\Lambda^{e}\right)^{\text {op }}\right)$ for the structure given in 5.2, part (3). This structure is precisely the one given in 5.5.1.1. This proves the proposition.

5.5.2. The description of $\operatorname{RHom}_{\Lambda^{e}}\left(\Lambda, \Lambda^{e}\right)$ made in 5.5.1 gets simpler when $H$ satisfies the left Artin-Schelter condition. Indeed, keep the setting of 5.5.1 and assume that there exists a natural integer $d$ such that $\operatorname{dim}_{\mathbb{k}} \operatorname{Ext}_{H}^{i}\left({ }_{H} \mathbb{k}, H\right)$ equals 1 if $i=d$ and 0 otherwise. The left homological integral is $\int_{\ell}=\operatorname{Ext}_{H}^{d}\left({ }_{H} \mathbb{k}, H\right)$. Also, denote by $\int_{\ell}: H \rightarrow \mathbb{k}$ the algebra homomorphism such that the right $H$-module structure of $\operatorname{Ext}_{H}^{d}\left({ }_{H} \mathbb{k}, H\right)$ is given by $\alpha<h=\int_{\ell}(h) \alpha$. Let $D_{A} \in \mathcal{C}\left(\Delta_{1}\right)$ be such that $D_{A} \simeq \operatorname{RHom}_{A^{e}}\left(A, A^{e}\right)$ in $\mathcal{D}\left(\left(A^{e}\right)^{\mathrm{op}}\right)$. Given that $\Xi_{\int_{e} \mathrm{o} S^{-1}}^{r}=\left(\Xi_{\int_{e}}^{r}\right)^{-1}$, the conclusion of 5.5.1 entails that $\operatorname{RHom}_{\Lambda^{e}}\left(\Lambda, \Lambda^{e}\right) \simeq D_{A} \otimes H[-d]$ in $\mathcal{D}\left(\left(\Lambda^{e}\right)^{\mathrm{op}}\right)$, where $D_{A} \otimes H[-d]$ has structure of $\operatorname{dg} \Lambda$-bimodule given by

$$
\begin{aligned}
& a(d \otimes \ell) b=\left(a d\left(\ell_{1} \rightarrow b\right)\right) \otimes \ell_{2} \\
& h(d \otimes \ell) k=\left(h_{1} \rightarrow d\right) \otimes\left(S^{-2} \circ \Xi_{\int_{\ell}}^{r}\right)^{-1}\left(h_{2}\right) \ell k .
\end{aligned}
$$

To sum up:

Corollary. Let $H$ be a Hopf algebra with Van den Bergh duality in dimension $d$ (and hence with invertible antipode $S$, see 3.4.2). Let $A$ be an $H$-module $d g$ algebra. Assume that $A$ is homologically smooth and let $D_{A} \in \mathcal{C}\left(\Delta_{1}\right)$ be such that $D_{A} \simeq \operatorname{RHom}_{A^{e}}\left(A, A^{e}\right)$ in $\mathcal{D}\left(\left(A^{e}\right)^{\mathrm{op}}\right)$ (see 5.4). Then, $\Lambda$ is homologically smooth and $\operatorname{RHom}_{\Lambda^{e}}\left(\Lambda, \Lambda^{e}\right) \simeq D_{A \sharp}\left(S^{-2} \circ \Xi_{\int_{\ell}}^{r}\right)^{-1} H[-d]$ in $\mathcal{D}\left(\left(\Lambda^{e}\right)^{\mathrm{op}}\right)$.

\section{Application to Constructions of Calabi-Yau algebras}

This section describes the deformed Calabi-Yau completions of $\Lambda$. The undeformed case is treated in Section 6.1 and the general case is treated in Section 6.2. Section 6.3 makes a specialisation to the case where $H$ is involutive and Calabi-Yau.

Given a homologically smooth $\operatorname{dg}$ algebra $A$ and an integer $n \in \mathbb{Z}$, the CalabiYau completion $\Pi_{n}(A)$ is the dg algebra $T_{A}\left(D_{A}[n-1]\right)$, where $D_{A}$ is any cofibrant replacement of $\mathrm{RHom}_{A^{e}}\left(A, A^{e}\right)$ in $\mathcal{C}\left(\left(A^{e}\right)^{\text {op }}\right)$. Every $\alpha \in \mathrm{HH}_{n-2}(A)$ yields a deformation $\Pi_{n}(A, \alpha)$ called a deformed Calabi-Yau completion of $A$ : Since $\mathrm{HH}_{n-2}(A) \simeq H^{0} \operatorname{Hom}_{A^{e}}\left(D_{A}[n-1], A[1]\right)$, then $\alpha$ is represented by some 0 -cocycle $c: D_{A}[n-1] \rightarrow A[1]$; Then, $\Pi_{n}(A, \alpha)$ is the unique dg algebra such that

- it has the same underlying graded algebra as $\Pi_{n}(A)$,

- its differential extends the one of $A$,

- if $d_{A} \in D_{A}[n-1]$, then its differential in $\Pi_{n}(A, \alpha)$ is the sum of its differential in $D_{A}[n-1]$ and of $c\left(d_{A}\right)$. 
$\Pi_{n}(A)$ is $n$-Calabi-Yau (see [21]), and so is $\Pi_{n}(A, \alpha)$ if $A$ is finitely cellular and $c$ lifts to the negative cyclic homology of $A$ (see [39]).

In this section, $H$ is a Hopf algebra with Van den Bergh duality in dimension $d$ and $A$ is a homologically smooth $H$-module $\operatorname{dg}$ algebra. Denote $A \sharp H$ by $\Lambda$. Let $n \in \mathbb{Z}$. Let $\sigma=S^{2} \circ \Xi_{\int_{\ell} \circ S}^{r}$. Let $D_{A}$ be a cofibrant left $\operatorname{dg} \Delta_{1}$-module such that $D_{A} \simeq \operatorname{RHom}_{A^{e}}\left(A, A^{e}\right)$ in $\mathcal{D}\left(A^{e}\right)$. In particular, $D_{A}$ is cofibrant in $\mathcal{C}\left(A^{e}\right)$ (see the proof in 5.3.2). Until the end of the section, $\Pi_{n}(A)$ and $T_{A}\left(D_{A}[n-1]\right)$ are identified.

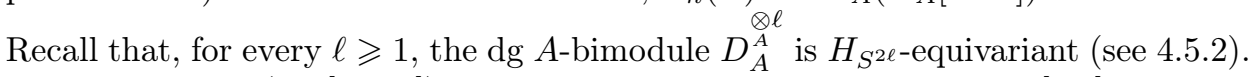
In particular, $T_{A}\left(D_{A}[n-1]\right)$ is a left $\operatorname{dg} H$-module. Denote $D_{A} \sharp^{\sigma} H[-d]$ by $D_{\Lambda}$.

Lemma. $D_{\Lambda}$ is a cofibrant replacement of $\operatorname{RHom}_{\Lambda^{e}}\left(\Lambda, \Lambda^{e}\right)$ in $\mathcal{C}\left(\Lambda^{e}\right)$.

Proof. Following 5.5.2, it is sufficient to prove that $D_{A} \sharp^{\sigma} H$ is cofibrant in $\mathcal{C}\left(\Lambda^{e}\right)$. Since $D_{A}$ is cofibrant in $\mathcal{C}\left(\Delta_{1}\right)$, this follows from the isomorphism proved in 4.2 .2

Until the end of the section, $\Pi_{n+d}(\Lambda)$ and $T_{\Lambda}\left(D_{\Lambda}[n+d-1]\right)$ are identified. Note that $D_{\Lambda}[n+d-1]=D_{A} \sharp^{\sigma} H[n-1]$.

6.1. The Calabi-Yau completion of $A \sharp H$. The dg algebra $\Pi_{n}(A) \sharp \sigma^{*} H$ was defined in 4.5.2. It has $T_{A}\left(D_{A}[n-1]\right) \otimes H$ as underlying complex, it contains $A \sharp H$ as a dg subalgebra, and its product is given by 4.5.2.2.

Proposition. (1) There is an isomorphism of $d g$ algebras $\Pi_{n}(A) \sharp \sigma^{*} H \stackrel{\sim}{\longrightarrow}$ $\Pi_{n+d}(A \sharp H)$.

(2) If $H$ is involutive and Calabi-Yau, then $\Pi_{n}(A)$ is an $H$-module dg algebra and $\Pi_{n}(A) \sharp H \simeq \Pi_{n+d}(A \sharp H)$.

Proof. (1) This follows from the considerations in 4.5 .2

(2) Following 3.5.3. $\int_{\ell}=\epsilon$, and hence $\sigma=\operatorname{Id}_{H}$. Thus (2) follows from (1).

Note that the isomorphism $\Pi_{n}(A) \sharp \sigma^{*} H \rightarrow \Pi_{n+d}(A \sharp H)$ in the previous result is uniquely determined by the following properties

- it extends the identity mappings $A \rightarrow A$ and $H \rightarrow H$,

- it maps every $d_{A} \in D_{A}[n-1]$ to $d_{A} \otimes 1 \in D_{\Lambda}[n+d-1]$.

6.2. Deformed Calabi-Yau completions of $A \sharp H$. This section gives some descriptions of the deformed Calabi-Yau completions of $A \sharp H$.

6.2.1. For this purpose, the following lemma relates the cohomology classes in $\mathrm{HH}_{n+d-2}(\Lambda)$ to Hochschild cohomology classes of $A$.

Lemma. The mapping $c \mapsto c_{\mid D_{A} \otimes 1}$ yields an isomorphism from $\operatorname{Hom}_{\Lambda^{e}}\left(D_{A} \sharp \sigma, \Lambda\right)$ to the subcomplex of $\operatorname{Hom}_{A^{e}}\left(D_{A}, \Lambda\right)$ consisting of those $c$ satisfying the identity

$$
c(h \rightarrow d)=h_{1} c(d) \int_{\ell}\left(h_{2}\right) S^{3}\left(h_{3}\right) .
$$

Proof. Note that every $\operatorname{dg} \Lambda^{e}$-module is a $\mathrm{dg} \Delta_{1}$-module by restriction-of-scalars along 4.1.0.3. By adjunction, there is an isomorphism

$$
\operatorname{Hom}_{\Lambda^{e}}\left(D_{A} \sharp^{\sigma} H, \Lambda\right) \simeq \operatorname{Hom}_{\Delta_{1}}\left(D_{A}, \operatorname{Hom}_{\Lambda^{e}}\left(\Lambda \otimes{ }^{\varphi} \Lambda, \Lambda\right)\right),
$$

where $\varphi \in \operatorname{Aut}_{\mathrm{dg}-\mathrm{alg}}(\Lambda)$ is defined by $\varphi(a h)=a \sigma\left(S^{-2}(h)\right)$ (see 4.2.2). Moreover, the $\operatorname{dg} \Lambda^{e}$-module $\operatorname{Hom}_{\Lambda^{e}}(\Lambda \otimes \varphi \Lambda, \Lambda)$ identifies with $\Lambda^{\varphi}$. Finally, 4.3 yields that $\operatorname{Hom}_{\Delta_{1}}\left(D_{A}, \Lambda^{\varphi}\right)$ is the subcomplex of $\operatorname{Hom}_{A^{e}}\left(D_{A}, \Lambda\right)$ consisting of those $c$ satisfying the identity

$$
c(h \rightarrow d)=h_{1} c(d) \varphi\left(S^{3}\left(h_{2}\right)\right) .
$$

Note that $\varphi\left(S^{3}(h)\right)=\sigma(S(h))=S^{3}\left(h_{2}\right) \int_{\ell}\left(S^{2}\left(h_{1}\right)\right)=S^{3}\left(h_{2}\right) \int_{\ell}\left(h_{1}\right)$. Whence the conclusion of the lemma. 
Note that the inverse of the isomorphism established in the previous lemma maps every $c$ to the morphism $D_{A} \sharp^{\sigma} H \rightarrow \Lambda, d \otimes \ell \mapsto c(d) \ell$. Moreover, this lemma identifies $\mathrm{HH}_{n+d-2}(\Lambda)$ with the 0-th cohomology space of the subcomplex of $\operatorname{Hom}_{A^{e}}\left(D_{A}[n-1], \Lambda[1]\right)$ consisting of those morphisms $c$ satisfying the identity 6.2.1.1.

6.2.2. In general, a deformed Calabi-Yau completion of $A \sharp H$ may not be expressed in terms of one of $A$. Instead, it may be related to a deformation of $\Pi_{n}(A) \sharp \sigma^{*} H$. This deformation is determined by the cocycle introduced by the following lemma. Let $\alpha \in \mathrm{HH}_{n+d-2}(\Lambda)$ be represented by $c \in Z^{0} \operatorname{Hom}_{A^{e}}\left(D_{A}[n-1], \Lambda[1]\right)$ (in the sense of 6.2.1 satisfying the identity 6.2.1.1.

Lemma. There exists a unique degree 1 square-zero derivation $\partial$ on the graded algebra $\Pi_{n}(A) \sharp^{\sigma^{*}} H$ such that

(a) $\partial_{\mid \Lambda}$ is the differential of $\Lambda$,

(b) $\partial_{\mid D_{A}[n-1]}$ takes its values in $\Lambda[1] \oplus D_{A}[n]$ and is equal to the sum of $c: D_{A}[n-$ $1] \rightarrow \Lambda[1]$ and the differential of $D_{A}[n-1]$.

Proof. Since the graded algebra $\Pi_{n}(A) \sharp \sigma^{*} H$ is generated by $\Lambda$ and $D_{A}$, the given conditions force $\partial$ to be unique when it exists. Note the following identities in $\Lambda$

$$
\begin{aligned}
c\left(h_{1} \rightarrow d\right) \sigma\left(h_{2}\right) & =h_{1} c(d) \int_{\ell}\left(h_{2}\right) S^{3}\left(h_{3}\right) \sigma\left(h_{4}\right) \\
& =h_{1} c(d) \int_{\ell}\left(h_{2}\right) S^{3}\left(h_{3}\right) S^{2}\left(h_{4}\right) \int_{\ell}\left(S\left(h_{5}\right)\right) \\
& =h c(d) .
\end{aligned}
$$

Given that $c$ is $A^{e}$-linear and that $\sigma$ satisfies the identity $\sigma(h)_{1} \otimes \sigma(h)_{2}=S^{2}\left(h_{1}\right) \otimes$ $\sigma\left(h_{2}\right)$, these considerations entail that there exists a degree 1 derivation $\partial$ on the graded algebra $\Pi_{n}(A) \sharp \sigma^{*} H$ satisfying (a) and (b). Since $c$ is a cocycle, then $\partial^{2}=$ 0 .

6.2.3. The following proposition expresses the deformed Calabi-Yau completions of $A \sharp H$ as deformations of $\Pi_{n}(A) \sharp \sigma^{*} H$.

Proposition. Let $\alpha \in \mathrm{HH}_{n+d-2}(\Lambda)$ be represented by the cocycle $D_{\Lambda}[n+d-1] \rightarrow$ $\Lambda[1], d \otimes \ell \mapsto c(d) \otimes \ell$ where $c \in Z^{0} \operatorname{Hom}_{A^{e}}\left(D_{A}[n-1], \Lambda[1]\right)$ satisfies the identity 6.2.1.1. Then, $\Pi_{n+d}(A \sharp H, \alpha) \simeq\left(\Pi_{n}(A) \sharp \sigma^{*} H, \partial\right)$ as dg algebras, where $\partial$ is as in 6.2.2.

Proof. Following 6.1 there exists an isomorphism of dg algebras $\varphi: T_{A}\left(D_{A}[n-\right.$ $1]) \sharp \sigma^{*} H \rightarrow T_{\Lambda}\left(D_{\Lambda}[n+d-1]\right)$ extending the identity mappings $A \rightarrow A$ and $H \rightarrow H$ and mapping every $d_{A} \in D_{A}[n-1]$ to $d_{A} \otimes 1 \in D_{\Lambda}[n+d-1]$. Comparing the differentials of $d_{A}$ and $d_{A} \otimes 1$ in $\left(\Pi_{n}(A) \sharp \sigma^{*} H, \partial\right)$ and $\Pi_{n+d}(A \sharp H, \alpha)$, respectively, yields that $\varphi$ is also an isomorphism of $d g$ algebras $\left(\Pi_{n}(A) \sharp \sigma^{*} H, \partial\right) \rightarrow \Pi_{n+d}(A \sharp H, \alpha)$.

6.3. Smash products with involutory Hopf algebras. Assume that $S^{2}=\operatorname{Id}_{H}$ and $H$ is Calabi-Yau. In this particular case, the following result shows that every deformed Calabi-Yau completion of $A$ is an $H$-module dg algebra, and that the resulting smash product is a deformed Calabi-Yau completion of $A \sharp H$.

Indeed, $\int_{\ell}=\epsilon($ see 3.5.3 $)$ and $\sigma=\operatorname{Id}_{H}$. Then, any $H$-linear cocycle $c: D_{A}[n-$ $1] \rightarrow A[1]$ may be considered as lying in $Z^{0} \operatorname{Hom}_{A^{e}}\left(D_{A}[n-1], \Lambda[1]\right)$ and, as such, satisfies the identity 6.2.1.1); In such a situation, the corresponding cocycle (see 6.2.1) in $\operatorname{Hom}_{\Lambda^{e}}\left(D_{\Lambda}[n+d-1], \Lambda[1]\right)$ is denoted by $\bar{c}$.

Proposition. Let $H$ be an involutive Hopf algebra which is moreover Calabi-Yau in dimension $d$. Let $A$ be a homologically smooth $H$-module dg algebra. Let $n \in \mathbb{Z}$. Let $c \in Z^{0} \operatorname{Hom}_{A^{e}}\left(D_{A}[n-1], A[1]\right)$ be H-linear. Denote by $\alpha \in \mathrm{HH}_{n-2}(A)$ and $\bar{\alpha} \in \mathrm{HH}_{n+d-2}(\Lambda)$ the cohomology classes of $c$ and $\bar{c}$, respectively. Then $\Pi_{n}(A, \alpha)$ is an $H$-module dg algebra and $\Pi_{n}(A, \alpha) \sharp H \simeq \Pi_{n+d}(A \sharp H, \bar{\alpha})$. 
Proof. Denote by $\delta$ the differential of $D_{A}[n-1]$. Hence, $\delta \otimes \operatorname{Id}_{H}$ is the differential of $D_{\Lambda}[n+d-1]$. Note that $\bar{c}$ is given by $\bar{c}\left(d_{A} \otimes \ell\right)=c\left(d_{A}\right) \otimes \ell$ for every $d_{A} \otimes \ell \in D_{\Lambda}$.

The dg algebra $\Pi_{n}(A, \alpha)$ is uniquely determined by the following properties

- it has the same underlying graded algebra as $\Pi_{n}(A)$,

- its differential extends the one of $A$,

- if $d_{A} \in D_{A}[n-1]$, then its differential is $\delta\left(d_{A}\right)+c\left(d_{A}\right)$.

Since $\Pi_{n}(A)$ is an $H$-module dg algebra (see 6.1. part (2)) and since $c$ is $H$-linear, then $\Pi_{n}(A, \alpha)$ is an $H$-module dg algebra.

The dg algebra $\Pi_{n+d}(A \sharp H, \bar{\alpha})$ is uniquely determined by the following properties

- it has the same underlying graded algebra as $\Pi_{n+d}(A \sharp H)$,

- its differential extends the one of $A \sharp H$,

- if $d_{A} \otimes \ell \in D_{\Lambda}[n+d-1]$, then its differential is $\left(\delta \otimes \operatorname{Id}_{H}\right)\left(d_{A} \otimes \ell\right)+\bar{c}\left(d_{A} \otimes \ell\right)=$ $\delta\left(d_{A}\right) \otimes \ell+c\left(d_{A}\right) \otimes \ell$.

Now, the isomorphism of $\mathrm{dg}$ algebras $\Pi_{n}(A) \sharp H \rightarrow \Pi_{n+d}(A \sharp H)$ of 6.1 (part (2)) extends the identity mappings $A \rightarrow A$ and $H \rightarrow H$ and it maps every $d_{A} \in$ $D_{A}[n-1]$ to $d_{A} \otimes 1 \in D_{\Lambda}[n+d-1]$. Hence, it also is an isomorphism of dg algebras $\Pi_{n}(A, \alpha) \sharp H \rightarrow \Pi_{n+d}(A \sharp H, \bar{\alpha})$.

\section{Application to Van den Bergh and Calabi-Yau duality}

Assume that $A$ is a $\mathbb{k}$-algebra. This section studies when $\Lambda=A \sharp H$ has Van den Bergh duality (in Section 7.1) or is skew Calabi-Yau (in Section 7.3). In the latter case a Nakayama automorphism is given for $\Lambda$. This is based on the notion of weak homological determinant given in Section 7.2 This notion extends the definition of homological determinant when the latter is not defined properly. Finally, a characterisation of when $\Lambda$ is Calabi-Yau is given in Section 7.4 when $A$ is connected $(\mathbb{N}$-)graded and $H$ is Calabi-Yau.

7.1. The inverse dualising bimodule. Following 5.4 when the antipode of $H$ is invertible, the cohomology space $\operatorname{Ext}_{A^{e}}^{i}\left(A, A^{e}\right)$ is an $H_{S^{2}}$-equivariant $A$-bimodule (equivalently a left $\Delta_{1}$-module). Recall (3.5.1) that a Hopf algebra with Van den Bergh duality has an invertible antipode. Note that in the particular case where $H$ is Calabi-Yau, it is proved in [12, Theorem 17] that if $A$ has Van den Bergh duality then so does $\Lambda$.

Proposition. Let $A$ be an $H$-module algebra where $H$ is a Hopf algebra. Assume that the antipode $S$ is invertible and that both $A$ and $H$ are homologically smooth. Then, the following assertions are equivalent

(i) $A$ and $H$ have Van den Bergh duality,

(ii) $\Lambda$ has Van den Bergh duality.

When these conditions are satisfied and $n, d$ are the corresponding homological dimensions of $A$ and $H$, respectively, then $\Lambda$ has dimension $n+d$ and

$$
\operatorname{Ext}_{\Lambda^{e}}^{n+d}\left(\Lambda, \Lambda^{e}\right) \simeq \operatorname{Ext}_{A^{e}}^{n}\left(A, A^{e}\right) \sharp{ }^{\left(S^{-2} \circ \Xi_{\int_{\ell}}^{r}\right)^{-1}} H .
$$

Proof. The implication $(i) \Rightarrow(i i)$ follows from 5.5 .2 and 4.6 .1 .

Conversely, assume that $\Lambda$ has Van den Bergh duality. There exist maximal integers $n$ and $d$ such that $\operatorname{Ext}_{A^{e}}^{n}\left(A, A^{e}\right)$ and $\operatorname{Ext}_{H}^{d}\left({ }_{H} \mathbb{k}, H\right)$ are nonzero. Denote $\operatorname{Ext}_{A^{e}}^{n}\left(A, A^{e}\right), \operatorname{Ext}_{H}^{d}\left({ }_{H} \mathbb{k}, H\right)$ and $\operatorname{Ext}_{\Lambda^{e}}^{n+d}\left(\Lambda, \Lambda^{e}\right)$ by $D_{A}, E$ and $D_{\Lambda}$, respectively. The proof that $A$ has Van den Bergh duality proceeds according the following steps

(a) $\Lambda$ has Van den Bergh duality in dimension $n+d$, moreover $\operatorname{Ext}_{A^{e}}^{i}\left(A, A^{e}\right) \neq 0$ if and only if $i=n$, and $\operatorname{Ext}_{H}^{j}\left({ }_{H} \mathbb{k}, H\right) \neq 0$ if and only if $j=d$, 
(b) given any left $\Lambda$-module $M$, there exists an isomorphism of left $\Lambda$-modules $D_{\Lambda} \underset{\Lambda}{\otimes} M \simeq\left(D_{A} \otimes_{A} M\right) \otimes E$ where the module structure of the right handside term is given by $a(d \otimes m \otimes e)=a d \otimes m \otimes e$ and $h(d \otimes m \otimes e)=h_{1} \rightarrow$ $d \otimes S^{2}\left(h_{2}\right) m \otimes e<S^{-1}\left(h_{3}\right)$,

(c) $H$ has Van den Bergh duality,

(d) $A$ has Van den Bergh duality.

Step (a) - Taking cohomology in the description of $\operatorname{RHom}_{\Lambda^{e}}\left(\Lambda^{e}, \Lambda\right)$ (5.5.1) shows that $n+d$ is the largest natural integer such that $\operatorname{Ext}_{\Lambda^{e}}^{n+d}\left(\Lambda, \Lambda^{e}\right) \neq 0$. Therefore, $\Lambda$ has Van den Bergh duality in dimension $n+d$. The same consideration then shows that $\operatorname{Ext}_{A^{e}}^{i}\left(A, A^{e}\right)=0$ if $i \neq n$ and $\operatorname{Ext}_{H}^{j}(H \mathbb{k}, H)=0$ if $j \neq d$. Using 5.5.1. the $\Lambda$-bimodule $D_{\Lambda}$ is identified with $D_{A} \otimes H \otimes E$ for the structure of $\Lambda$-bimodule described in (5.5.1.1).

Step (b) - In view of the structure of right $\Lambda$-module of $D_{\Lambda}=D_{A} \otimes H \otimes E$, there are well-defined linear mappings $\Phi:\left(D_{A} \otimes \otimes_{A} M\right) \otimes E \rightarrow D_{\Lambda} \otimes_{\Lambda} M$ and $\Psi: D_{\Lambda} \otimes{ }_{\Lambda} M \rightarrow$ $\left(D_{A} \otimes M\right) \otimes E$ given by $\Phi(d \otimes m \otimes e)=(d \otimes 1 \otimes e) \otimes m$ and $\Psi(d \otimes \ell \otimes e) \otimes m=d \otimes \ell m \otimes e$. Note the identity $(d \otimes \ell \otimes e) \otimes m=(d \otimes 1 \otimes e) \otimes \ell m$ in $D_{\Lambda} \otimes M$. Therefore $\Phi$ and $\Psi$ are inverse to each other. The claim of Step (b) is therefore proved by transporting the left $\Lambda$-module structure of $D_{\Lambda} \otimes_{\Lambda} M$ to $\left(D_{A}{\underset{A}{Q}}_{A} M\right) \otimes E$ using $\Phi$ and $\Psi$.

Step (c) - Let $M=D_{\Lambda}^{-1} \otimes A \in \bmod (\Lambda)$. From the previous step, it follows that $A \simeq\left(D_{A} \otimes M\right)^{\left(\operatorname{dim}_{\mathrm{k}} E\right)}$ in $\bmod (A)$. Thus, $\operatorname{dim}_{\mathbb{k}} E<\infty$. Using 3.4.1, 3.5.1 and step (a) yields that $H$ has Van den Bergh duality in dimension $d$.

Step (d) - It suffices to prove that $D_{A}$ is invertible as an $A$-bimodule. Since $H$ has Van den Bergh duality, it follows from 5.5.2 that $D_{\Lambda} \simeq D_{A \sharp}{ }^{\sigma} H$ where $\sigma=\left(S^{-2} \circ \Xi_{\int_{\ell}}^{r}\right)^{-1}$. Now, applying 4.6 .2 yields the desired conclusion.

More explicitly, $\operatorname{Ext}_{\Lambda^{e}}^{n+d}\left(\Lambda, \Lambda^{e}\right)$ is isomorphic to the vector space $\operatorname{Ext}_{A^{e}}^{n}\left(A, A^{e}\right) \otimes$ $H$ endowed with the following structure of $\Lambda$-bimodule:

$$
\begin{aligned}
a(d \otimes \ell) b & =\left(a d\left(\ell_{1} \rightarrow b\right)\right) \otimes \ell_{2} \\
h(d \otimes \ell) k & =\left(h_{1} \rightarrow d\right) \otimes\left(S^{-2} \circ \Xi_{\int_{\ell}}^{r}\right)^{-1}\left(h_{2}\right) \ell k .
\end{aligned}
$$

7.2. (Weak) homological determinants. Assume that the antipode of $H$ is invertible, that $A$ has Van den Bergh duality in dimension $n$, and that $\operatorname{Ext}_{A^{e}}^{n}\left(A, A^{e}\right)$ is free of $\operatorname{rank}$ one in $\bmod (A)$ (for instance, $A$ is skew Calabi-Yau). Weak homological determinants are introduced here to express a Nakayama automorphism for $\Lambda$. When the homological determinant is well-defined (like in [23, 18, 30]), the two notions coincide.

Fix a free generator $e_{A}$ of the left $A$-module $\operatorname{Ext}_{A^{e}}^{n}\left(A, A^{e}\right)$. It determines a unique algebra homomorphism $\mu_{A}: A \rightarrow A$ such that the following identity holds in $\operatorname{Ext}_{A^{e}}^{n}\left(A, A^{e}\right): e_{A} a=\mu_{A}(a) e_{A}$. When $A$ happens to be skew Calabi-Yau in dimension $n$, then $\mu_{A}$ is a Nakayama automorphism of $A$.

Denote by $f_{A}$ the morphism of left $A$-modules $\operatorname{Ext}_{A^{e}}^{n}\left(A, A^{e}\right) \rightarrow A$ defined by $f_{A}\left(e_{A}\right)=1$. Since $\operatorname{Ext}_{A^{e}}^{n}\left(A, A^{e}\right)$ is an $H_{S^{2}}$-equivariant $A$-bimodule (see 4.3, part (5)), then $\operatorname{Hom}_{A}\left(\operatorname{Ext}_{A^{e}}^{n}\left(A, A^{e}\right), A\right)$ is an $H_{S^{-2}}$-equivariant $A$-bimodule in the sense of 4.4.1 (part (1)).

This setting is assumed until the end of the section.

Example. Consider the situation of the example in 4.1. Keep the notation and material introduced there and in 5.4 . Then $A=\mathbb{k}\left[x_{1}, \ldots, x_{n}\right]$ is $n$-Calabi-Yau and 
$e_{A}$ may be taken equal to the cohomology class of the cochain

$$
\begin{aligned}
\varphi_{A}: \quad A \otimes \Lambda^{n} V \otimes A & \rightarrow A^{e} \\
a \otimes x_{1} \wedge \cdots \wedge x_{n} \otimes b & \mapsto a \otimes b .
\end{aligned}
$$

The Nakayama automorphism of $A$ corresponding to $e_{A}$ is $\operatorname{Id}_{A}$. Indeed, for a given $i \in\{1, \ldots, n\}$, the coordinate cochain

$$
\varphi_{i}: A \otimes \Lambda^{n-1} V \otimes A \rightarrow A^{e}
$$

associated with the term $1 \otimes x_{1} \wedge \cdots \widehat{x}_{i} \cdots \wedge x_{n} \otimes 1$ of the canonical basis of $A \otimes$ $\Lambda^{n-1} V \otimes A$ has coboundary $(-1)^{n-1} \varphi_{i} \circ d_{K}$, which is given by

$$
\begin{array}{ccc}
A \otimes \Lambda^{n} V \otimes A & \rightarrow & A \otimes A \\
1 \otimes x_{1} \wedge \cdots \wedge x_{n} \otimes 1 & \mapsto & (-1)^{n+i}\left(x_{i} \otimes 1-1 \otimes x_{i}\right) ;
\end{array}
$$

This is $(-1)^{n+i}\left(\varphi_{A} x_{i}-x_{i} \varphi_{A}\right)$; Therefore $\varphi_{A} x_{i}$ and $x_{i} \varphi_{A}$ are cohomologous, and hence $e_{A} x_{i}=x_{i} e_{A}$.

Given $X \in \mathfrak{g}$, then $X \rightarrow e_{A}$ is represented by $\partial_{X}\left(\varphi_{A}\right)$ which is such that (see $(5.4 .0 .2)$

$$
\begin{aligned}
\partial_{X}\left(\varphi_{A}\right)\left(1 \otimes x_{1} \wedge \cdots \wedge x_{n} \otimes 1\right) & =-\varphi_{A}\left(\partial_{X}\left(1 \otimes x_{1} \wedge \cdots \wedge x_{n} \otimes 1\right)\right) \\
& =-\sum_{i} \partial_{X}\left(x_{i}\right)_{i}^{\prime} \otimes \partial_{X}\left(x_{i}\right)_{i}^{\prime \prime}
\end{aligned}
$$

Thus, $X \rightarrow e_{A}=-\sum_{i} \partial_{X}(v)_{i}^{\prime \prime} \partial_{X}(v)_{i}^{\prime} e_{A}$ and, writing div for the usual divergence,

$$
X \rightarrow e_{A}=-\operatorname{div}\left(\partial_{X}\right) e_{A} .
$$

7.2.1. The definition of the weak homological determinant is made possible by the following technical result.

Lemma. In the setting introduced previously,

(1) $f_{A}$ is a free generator of the right $A$-module $\operatorname{Hom}_{A}\left(\operatorname{Ext}_{A^{e}}^{n}\left(A, A^{e}\right), A\right)$,

(2) Let $\lambda, w: H \rightarrow A$ be the mappings such that the following identities hold in $\operatorname{Ext}_{A^{e}}^{n}\left(A, A^{e}\right)$ and $\operatorname{Hom}_{A}\left(\operatorname{Ext}_{A^{e}}^{n}\left(A, A^{e}\right), A\right)$, respectively

$$
\left\{\begin{array}{l}
h \rightarrow e_{A}=\lambda(h) e_{A} \\
h \rightarrow f_{A}=f_{A} w(h) .
\end{array}\right.
$$

Then, they are connected by the following relations

$$
\left\{\begin{aligned}
w(h) & =S^{-2}\left(h_{2}\right) \rightarrow \lambda\left(S^{-3}\left(h_{1}\right)\right) \\
\lambda(h) & =h_{1} \rightarrow\left(w\left(S^{3}\left(h_{2}\right)\right)\right) .
\end{aligned}\right.
$$

(3) $\lambda$ satisfies the identity $\lambda(h k)=\left(h_{1} \rightarrow \lambda(k)\right) \lambda\left(h_{2}\right)$ in $A$.

(4) $w$ satisfies the identity $w(h k)=w\left(h_{1}\right)\left(S^{-2}\left(h_{2}\right) \rightarrow w(k)\right)$ in $A$.

Proof. (1) follows from the definition of $f_{A}$.

(2) Since $\operatorname{Ext}_{A^{e}}^{n}\left(A, A^{e}\right)$ is free of rank one in $\bmod (A)$, the mappings $\lambda$ and $\mu$ exist and are unique. The third equality is due to the following computation

$$
\begin{aligned}
& \left(h \rightarrow f_{A}\right)\left(e_{A}\right) \quad=\quad\left(f_{A} w(h)\right)\left(e_{A}\right)=f_{A}\left(e_{A}\right) w(h)=w(h) \\
& \begin{array}{l}
= \\
= \\
=
\end{array} \\
& S^{-2}\left(h_{2}\right) \rightarrow f_{A}\left(S^{-3}\left(h_{1}\right) \rightarrow e_{A}\right) \\
& =\quad S^{-2}\left(h_{2}\right) \rightarrow f_{A}\left(\lambda\left(S^{-3}\left(h_{1}\right)\right) e_{A}\right) \\
& =S^{-2}\left(h_{2}\right) \rightarrow \lambda\left(S^{-3}\left(h_{1}\right)\right) \text {. }
\end{aligned}
$$

The fourth equality is due to the following computation

$$
h_{1} \rightarrow w\left(S^{3}\left(h_{2}\right)\right)=h_{1} \rightarrow\left(S\left(h_{2}\right) \rightarrow \lambda\left(h_{3}\right)\right)=\lambda(h) .
$$

(3) The equality is due to the following computation,

$$
\begin{aligned}
(h k) \rightarrow e_{A} & =h \rightarrow\left(k \rightarrow e_{A}\right)=h \rightarrow\left(\lambda(k) e_{A}\right)=\left(h_{1} \rightarrow \lambda(k)\right)\left(h_{2} \rightarrow e_{A}\right) \\
& =\left(\left(h_{1} \rightarrow \lambda(k)\right) \lambda\left(h_{2}\right)\right) e_{A} .
\end{aligned}
$$


(4) The equality is due to the computation below

$$
\begin{aligned}
h \rightarrow\left(k \rightarrow f_{A}\right) & = & & (h k) \rightarrow f_{A}=f_{A} w(h k) \\
& = & & h \rightarrow\left(f_{A} w(k)\right) \\
& = & & \left(h_{1} \rightarrow f_{A}\right)\left(S^{-2}\left(h_{2}\right) \rightarrow w(k)\right) \\
4.4 .1 & \text { part (1) } & & \\
& = & & f_{A} w\left(h_{1}\right)\left(S^{-2}\left(h_{2}\right) \rightarrow w(k)\right) .
\end{aligned}
$$

7.2.2. Apply 7.2.1 and call the weak homological determinant associated with $e_{A}$ the linear mapping whdet: $H \rightarrow A$ which satisfies the following identity in $\operatorname{Hom}_{A}\left(\operatorname{Ext}_{A^{e}}^{n}\left(A, A^{e}\right), A\right)$

$$
h \rightarrow f_{A}=f_{A} \text { whdet }(h) .
$$

Whence the following identity in $\operatorname{Ext}_{A^{e}}^{n}\left(A, A^{e}\right)$

$$
h \rightarrow e_{A}=\left(h_{1} \rightarrow \operatorname{whdet}\left(S^{3}\left(h_{2}\right)\right)\right) e_{A} .
$$

The weak homological determinant is compatible with the multiplicative structure of $H$ and $A$ in the following sense

$$
\operatorname{whdet}(h k)=\operatorname{whdet}\left(h_{1}\right)\left(S^{-2}\left(h_{2}\right) \rightarrow \operatorname{whdet}(k)\right) .
$$

In view of expressing a Nakayama automorphism of $\Lambda$, denote by $\theta_{\text {whdet }}$ the mapping $H \rightarrow \Lambda$ defined by

$$
\theta_{\text {whdet }}(h)=\operatorname{whdet}\left(S^{2}\left(h_{1}\right)\right) h_{2} .
$$

According to 7.2 .2 .2 , this is an algebra homomorphism from $H$ to $\Lambda$.

Example. Consider the situation of the example in 4.1 and keep the notation and material introduced there, in 5.4 and in 7.2 . Then, the mapping $\lambda: H \rightarrow A$ of 7.2 .1 (part (2)) is given by $\lambda(1)=1$ and (

$$
(\forall X \in \mathfrak{g}) \quad \lambda(X)=-\operatorname{div}\left(\partial_{X}\right) .
$$

Therefore, whdet: $H \rightarrow A$ is given by $\operatorname{whdet}(1)=1$ and (see 7.2.1 part (2))

$$
(\forall X \in \mathfrak{g}) \quad \operatorname{whdet}(X)=\operatorname{div}(X) .
$$

And hence $\theta_{\text {whdet }}: H \rightarrow \Lambda$ is given by $\theta_{\text {whdet }}(1)=1$ and

$$
\theta_{\text {whdet }}(X)=\operatorname{div}\left(\partial_{X}\right)+X \text {. }
$$

7.2.3. Distinct choices for $e_{A} \in \operatorname{Ext}_{A^{e}}^{n}\left(A, A^{e}\right)$ may yield distinct weak homological determinants related to each other as follows (because $\operatorname{Hom}_{A}\left(\operatorname{Ext}_{A^{e}}^{n}\left(A, A^{e}\right), A\right.$ ) is an $H_{S^{-2} \text {-equivariant } A \text {-bimodule, see 4.4.1. }}$.

Lemma. Let $e_{A}^{\prime}$ be free generator of the left $A$-module $\operatorname{Ext}_{A^{e}}^{n}\left(A, A^{e}\right)$ and let whdet' be the associated weak homological determinant. Let $a_{0} \in A^{\times}$be such that $e_{A}^{\prime}=$ $a_{0} e_{A}$. Then, $\operatorname{whdet}^{\prime}(h)=a_{0} \operatorname{whdet}\left(h_{1}\right)\left(S^{-2}\left(h_{2}\right) \rightarrow a_{0}^{-1}\right)$ for all $h \in H$.

7.2.4. If $A$ is connected graded and $e_{A}$ is chosen to be homogeneous, then whdet is equal to the homological determinant already developed in [18, 23, 30]. Besides, distinct choices for $e_{A}$ (with the homogeneity requirement) yield the same homological determinants and the same Nakayama automorphisms of $A$. See $[30$, Definition 3.7 and Remark 3.8] for details, keeping in mind that $R^{d} \Gamma_{\mathfrak{m}_{A}}(A)^{*}$ there is $\operatorname{Hom}_{A}\left(\operatorname{Ext}_{A^{e}}^{n}\left(A, A^{e}\right), A\right)$ here.

In general, $\mathbb{k} \cdot e_{A}$ is an $H$-submodule of $\operatorname{Ext}_{A^{e}}^{n}\left(A, A^{e}\right)$ if and only if $\lambda$ and whdet take values in $\mathbb{k}$. In this case, and following the spirit of [30, Definition 3.7], the action of $H$ on $A$ is said to have a homological determinant and whdet is denoted by hdet. In particular, hdet: $H \rightarrow \mathbb{k}$ is an algebra homomorphism (see 77.2 .2 .2$)$ ), hdet $\circ S^{2}=$ hdet, the identity $h \rightarrow e_{A}=\operatorname{hdet}(S(h)) e_{A}$ holds in $\operatorname{Ext}_{A^{e}}^{n}\left(A, A^{e}\right)$, and $\theta_{\text {hdet }}=\Xi_{\text {hdet }}^{\ell}$. 
7.3. Nakayama automorphisms of $A \sharp H$. This section gives necessary and sufficient conditions for $\Lambda=A \sharp H$ to be skew Calabi-Yau. In such a case, it gives a Nakayama automorphism for $\Lambda$. Recall that the antipode is invertible as soon as $H$ has Van den Bergh duality (3.5.1).

7.3.1. The following result gives a sufficient condition for $\Lambda$ to be skew Calabi-Yau. Note that it was first established in [30, Theorem 4.1] assuming that $A$ is connected graded and $H$ is finite dimensional. See also [40] for similar conclusions about Hopf Galois objects of skew Calabi-Yau Hopf algebras.

Proposition. Let $A$ be an $H$-module algebra where $H$ is a Hopf algebra. Assume that $A$ and $H$ are skew Calabi-Yau in dimension $n$ and $d$, respectively. Then, $\Lambda=$ $A \sharp H$ is skew Calabi-Yau in dimension $n+d$ and admits a Nakayama automorphism $\mu_{\Lambda}$ given by

$$
\mu_{\Lambda}=\mu_{A} \sharp\left(\theta_{\text {whdet }} \circ \mu_{H}\right)
$$

where $\mu_{A}$ is the Nakayama automorphism of $A$ and whdet: $H \rightarrow A$ is the weak homological determinant associated to any generator of $\operatorname{Ext}_{A^{e}}^{n}\left(A, A^{e}\right)$ in $\bmod (A)$, and $\mu_{H}=S^{-2} \circ \Xi_{\int_{\ell}}^{r}$.

If, moreover, the action of $H$ on $A$ has a homological determinant (7.2.4), then

$$
\mu_{A \sharp H}=\mu_{A} \sharp\left(\Xi_{\text {hdet }}^{\ell} \circ \mu_{H}\right) \text {. }
$$

Proof. First, note (7.1) that $\Lambda$ has Van den Bergh duality in dimension $n+d$ and $\operatorname{Ext}_{\Lambda^{e}}^{n+d}\left(\Lambda, \Lambda^{e}\right) \simeq \operatorname{Ext}_{A^{e}}^{n}\left(A, A^{e}\right) \sharp \mu_{H}^{-1} H$. Let $e_{A} \in \operatorname{Ext}_{A^{e}}^{n}\left(A, A^{e}\right)$ be a free generator in $\bmod (A)$ satisfying the identity $e_{A} a=\mu_{A}(a) e_{A}$. Since $\operatorname{Ext}_{\Lambda^{e}}^{n+d}\left(\Lambda, \Lambda^{e}\right) \simeq$ $\operatorname{Ext}_{A^{e}}^{n}\left(A, A^{e}\right) \sharp \mu_{H}^{-1} H$ in $\bmod \left(\Lambda^{\mathrm{op}}\right)$, then $e_{A} \otimes 1$ is a free generator of $\operatorname{Ext}_{\Lambda^{e}}^{n+d}\left(\Lambda, \Lambda^{e}\right)$ in $\bmod \left(\Lambda^{\mathrm{op}}\right)$. Since $\operatorname{Ext}_{\Lambda^{e}}^{n+d}\left(\Lambda, \Lambda^{e}\right) \simeq H^{\mu_{H}} \sharp \operatorname{Ext}_{A^{e}}^{n}\left(A, A^{e}\right)$ in $\bmod (\Lambda)$ (see 4.2.3), then $e_{A} \otimes 1$ is also a free generator of $\operatorname{Ext}_{\Lambda^{e}}^{n+d}\left(\Lambda, \Lambda^{e}\right)$ in $\bmod (\Lambda)$. Hence, in order to prove the result, it suffices to show that the mapping $\mu_{\Lambda}: \Lambda \rightarrow \Lambda$ satisfies the following identity in $\operatorname{Ext}_{A^{e}}^{n}\left(A, A^{e}\right) \sharp^{\mu_{H}^{-1}} H$

$$
\left(e_{A} \otimes 1\right)(a h)=\mu_{\Lambda}(a h)\left(e_{A} \otimes 1\right) .
$$

This is done in the following computation

$$
\begin{array}{ccl}
\left(e_{A} \otimes 1\right)(a h) & = & \left(e_{A} a \otimes h\right) \\
= & \mu_{A}(a)\left(e_{A} \otimes h\right) \\
= & \mu_{A}(a) \mu_{H}\left(h_{2}\right)\left(S^{-3}\left(h_{1}\right) \rightarrow e_{A} \otimes 1\right) \\
\frac{1.2 .1 .2}{} & \\
= & \mu_{A}(a) \mu_{H}(h)_{2}\left(S^{-1}\left(\mu_{H}(h)_{1}\right) \rightarrow e_{A} \otimes 1\right) \\
= & \mu_{\Lambda}(a h)\left(e_{A} \otimes 1\right) .
\end{array}
$$

The description of $\mu_{A \sharp H}$ when the action of $H$ on $A$ has a homological determinant follows from the discussion in 7.2.4.

Example. Let $A=\mathbb{k}\left[x_{1}, \ldots, x_{n}\right]$ and let $\mathfrak{g}$ be a $d$-dimensional Lie algebra. Let $\mathfrak{g} \rightarrow \operatorname{Der}_{\mathfrak{k}}(A)$ be a Lie algebra homomorphism inducing an action of $H=\mathcal{U}(\mathfrak{g})$ on $A$. Denote by $\partial_{X}: A \rightarrow A$ the derivation associated with $X$ for every $X \in \mathfrak{g}$. Then, (see [38, Corollary 2.2] or (3.1.0.1) $\mu_{H}$ is given by

$$
\mu_{H}(X)=X+\operatorname{Tr}\left(\operatorname{ad}_{X}\right) .
$$

And $\Lambda=A \sharp H$ has a Nakayama automorphism $\mu_{\Lambda}: \Lambda \rightarrow \Lambda$ such that, for all $a \in A$ and $X \in \mathfrak{g}$ (see 7.2 and 7.2 .2 .5 )

$$
\begin{aligned}
\mu_{\Lambda}(a) & =a \\
\mu_{\Lambda}(X) & =X+\operatorname{div}\left(\partial_{X}\right)+\operatorname{Tr}\left(\operatorname{ad}_{X}\right) .
\end{aligned}
$$

Note that $\mu_{\Lambda}(X)$ need not lie in $H$. 
7.3.2. The following result is a partial converse to the implication proved in 7.3.1

Proposition. Let $H$ be a Hopf algebra with invertible antipode. Let $A$ be an $H$ module algebra. Assume that $A$ and $H$ are homologically smooth and that $A \sharp H$ is skew Calabi-Yau. Then, $H$ is skew Calabi-Yau. If, moreover, the action of $H$ on A has a homological determinant, then $A$ is skew Calabi-Yau as well.

Proof. Note that $A$ and $H$ have Van den Bergh duality, say in dimension $n$ and $d$, respectively, and hence the dimension of $\Lambda$ (as a skew Calabi-Yau algebra) is $n+d$ (see 7.1. In particular, $H$ is skew Calabi-Yau (see 3.5.1. Let $\mu_{H}=S^{-2} \circ \Xi_{\int_{\ell}}^{r}$. Then, $\operatorname{Ext}_{\Lambda^{e}}^{n+d}\left(\Lambda, \Lambda^{e}\right) \simeq \operatorname{Ext}_{A^{e}}^{n}\left(A, A^{e}\right) \sharp \mu_{H}^{-1} H$ in $\bmod \left(\Lambda^{e}\right)$ (see 7.1).

Applying $-\otimes A: \bmod (\Lambda) \rightarrow \bmod (A)$ to the free of rank one left $\Lambda$-module $\operatorname{Ext}_{\Lambda^{e}}^{n+d}\left(\Lambda, \Lambda^{e}\right)$ yields that $\operatorname{Ext}_{A^{e}}^{n}\left(A, A^{e}\right)$ is free of rank one in $\bmod (A)$ (see 7.1). Therefore, a weak homological determinant is defined (in the sense of 7.2.1).

Assume that a homological determinant hdet: $H \rightarrow \mathbb{k}$ exists (in the sense of 7.2.4), say associated to a free generator $e_{A} \in \operatorname{Ext}_{A^{e}}^{n}\left(A, A^{e}\right)$ in $\bmod (A)$. In particular, $\theta_{\text {hdet }}=\Xi_{\text {hdet }}^{\ell}$. Let $\mu_{A}: A \rightarrow A$ be the algebra homomorphism such that the identity $e_{A} a=\mu_{A}(a) e_{A}$ holds in $\operatorname{Ext}_{A^{e}}^{n}\left(A, A^{e}\right)$. In order to prove that $A$ is skew Calabi-Yau, it is convenient to prove that $\mu_{A}$ is an automorphism of $A$. For this purpose, the following arguments first prove that $\mu_{A} \sharp\left(\Xi_{\text {hdet }}^{\ell} \circ \mu_{H}\right)$ is an automorphism of $\Lambda$. Part of the considerations made in the proof of 7.3 .1 are valid in the present situation. In particular, $e_{A} \otimes 1$ is a free generator of $\operatorname{Ext}_{A^{e}}^{n}\left(A, A^{e}\right) \sharp^{\mu_{H}} H$ in $\bmod (\Lambda)$, and the following identity holds true (for $\lambda \in \Lambda$ )

$$
\left(e_{A} \otimes 1\right) \lambda=\left(\mu_{A} \sharp\left(\Xi_{\text {hdet }}^{\ell} \circ \mu_{H}\right)\right)(\lambda)\left(e_{A} \otimes 1\right) .
$$

Since $\Lambda$ is skew Calabi-Yau, the lemma in 2.3.2 applies to $D=\operatorname{Ext}_{\Lambda^{e}}^{n+d}\left(\Lambda, \Lambda^{e}\right) \simeq$ $\operatorname{Ext}_{A^{e}}^{n}\left(A, A^{e}\right) \sharp \mu_{H}^{-1} H$. It yields that $\mu_{A} \sharp\left(\Xi_{\text {hdet }}^{\ell} \circ \mu_{H}\right): \Lambda \rightarrow \Lambda$ is an automorphism. In particular

- $\mu_{A}$ is an injective mapping,

- composing with $1 \otimes \epsilon: \Lambda \rightarrow A$ yields that $\mu_{A}$ is a surjective mapping.

Thus, $\mu_{A} \in \operatorname{Aut}_{\mathrm{k}-\mathrm{alg}}(A)$. Since the mapping

$$
\begin{aligned}
A^{\mu_{A}} & \rightarrow \operatorname{Ext}_{A^{e}}^{n}\left(A, A^{e}\right) \\
a & \mapsto a e_{A}
\end{aligned}
$$

is an isomorphism in $\bmod \left(A^{e}\right)$, then $A$ is skew Calabi-Yau.

7.4. When is $A \sharp H$ Calabi-Yau? The algebra $A$ is Calabi-Yau if and only if it is skew Calabi-Yau and any Nakayama automorphism is inner (equivalently, the identity map of $A$ is a Nakayama automorphism). Using 7.3 , this section gives necessary and sufficient conditions for $\Lambda$ to be Calabi-Yau. Unfortunately, the techniques used here require restrictive hypotheses on $A$, that is, $A$ is augmented or connected graded.

7.4.1. Recall that the augmented ideal of an augmented $H$-module algebra is always assumed to be an $H$-submodule.

Lemma. Assume that $A$ is an augmented $H$-module algebra. Denote by $p: A \rightarrow \mathbb{k}$ the augmentation. Keep the hypotheses made in the proposition in 7.3 .1 as well as the notation introduced there. Assume, moreover, that $\Lambda$ is Calabi-Yau. Then,

(1) $p\left(\operatorname{whdet}\left(h_{1}\right)\right) \int_{\ell}\left(h_{2}\right)=\epsilon(h)$ for all $h \in H$;

(2) if the action of $H$ admits a homological determinant, then $\operatorname{hdet}\left(h_{1}\right) \int_{\ell}\left(h_{2}\right)=$ $\epsilon(h)$ for all $h \in H$; 
(3) if $A$ is connected graded, then

$$
\left(\exists h_{A} \in H^{\times}\right)(\forall a \in A) \quad \mu_{A}(a)=h_{A} \rightarrow a \underset{i n \Lambda}{=} h_{A} a h_{A}^{-1} .
$$

Proof. (1) The Nakayama automorphism $\left(\mu_{\Lambda}\right)$ of $\Lambda$ given in 7.3.1 is inner because $\Lambda$ is Calabi-Yau. Hence, there exists $\lambda \in \Lambda^{\times}$such that the following identities hold in $\Lambda$

$$
\left\{\begin{array}{l}
\mu_{\Lambda}(h)=\theta_{\text {whdet }} \circ \mu_{H}(h)=\lambda h \lambda^{-1} \\
\mu_{\Lambda}(a)=\mu_{A}(a)=\lambda a \lambda^{-1}
\end{array}\right.
$$

Denote by $\mathfrak{M}$ the kernel of $p$. Since $\mathfrak{M} \otimes H$ is a two-sided ideal of $\Lambda$, there exist $k, k^{\prime} \in H$ such that $\lambda \in k+\mathfrak{M} \otimes H$ and $\lambda^{-1} \in k^{\prime}+\mathfrak{M} \otimes H$. Since $\lambda$ is invertible, then $k$ is invertible in $H$ and $k^{\prime}$ is its inverse. Let $h \in H$; Then, $\lambda h \lambda^{-1} \in k h k^{-1}+\mathfrak{M} \otimes H$; Consequently $(p \otimes \epsilon)\left(\lambda h \lambda^{-1}\right)=\epsilon(h)$; Now, 7.4.1.2 entails that $(p \otimes \epsilon)\left(\lambda h \lambda^{-1}\right)=$ $(p \otimes \epsilon) \circ \theta_{\text {whdet }} \circ \mu_{H}(h)$; Note that $(p \otimes \epsilon) \circ \theta_{\text {whdet }}=p \circ$ whdet $\circ S^{2}$ (see 7.2.2.3) ; Since $\mu_{H}=S^{-2} \circ \Xi_{\int_{\ell}}^{r}$, then $\epsilon(h)=p\left(\operatorname{whdet}\left(h_{1}\right)\right) \int_{\ell}\left(h_{2}\right)$.

(2) The additional hypothesis means that whdet takes its values in $\mathbb{k}$, and (by definition) hdet $=$ whdet. The conclusion therefore follows from (1).

(3) Assume that $A$ is connected graded. Then, there exists $\ell \in \mathbb{Z}$ such that $\operatorname{Ext}_{A^{e}}^{n}\left(A, A^{e}\right) \simeq A^{\mu_{A}}(\ell)$ as graded $A$-bimodules. In particular, $\mu_{A}: A \rightarrow A$ is homogeneous. Let $a \in A$ be homogeneous. Since $A$ is connected graded, then $\Lambda^{\times}=H^{\times}$, and hence $\mu_{A}(a)=k a k^{-1}=\left(k_{1} \rightarrow a\right) k_{2} k^{-1}$ in $\Lambda ;$ Applying $\operatorname{Id}_{A} \otimes \epsilon: \Lambda \rightarrow$ $A$ yields the equality $\mu_{A}(a)=\epsilon\left(k^{-1}\right) k \rightarrow a$. Thus 7.4.1.1 holds true taking $h_{A}=\epsilon\left(k^{-1}\right) k$.

Keep the setting of part (3) in the previous result. Since $\mu_{A}$ is homogeneous, then $\mu_{\Lambda}\left(A_{n} \otimes H\right)=A_{n} \otimes H$ for every $n \in \mathbb{N}$. Therefore, in the previous proof, one may assume that $\lambda=h_{A}$ when $A$ is connected graded.

7.4.2. The following result determines when $A \sharp H$ is Calabi-Yau assuming that $H$ is Calabi-Yau and $A$ is connected graded.

Theorem. Let $H$ be a Calabi-Yau Hopf algebra. Let $A$ be a connected graded $H$ module algebra. Let $h_{0} \in H^{\times}$be such that $S^{-2}$ is the inner automorphism of $h_{0}$ (see 3.5.3). Then, $\Lambda=A \sharp H$ is Calabi-Yau if and only if the following conditions hold

(a) A is skew Calabi-Yau,

(b) hdet $=\epsilon$,

(c) $\left(\exists k_{A} \in Z\left(H^{\times}\right)\right)(\forall a \in A) \quad \mu_{A}(a)=\left(h_{0} k_{A}\right) \rightarrow a \underset{\text { in } \Lambda}{=}\left(h_{0} k_{A}\right) a\left(h_{0} k_{A}\right)^{-1}$.

Proof. In order to prove the equivalence it may be assumed that $A$ is skew CalabiYau in the graded sense (see 7.2.4 7.3.1 7.3.2 and 2.3.3. Note that $\int_{\ell}=\epsilon$ due to 3.5.3 applied to $H^{\mathrm{op}}$.

Assume that $\Lambda$ is Calabi-Yau. Following 7.4.1 and the the final remark made there, hdet $=\epsilon$ and there exists $h_{A} \in H^{\times}$such that the following identities hold

$$
\begin{aligned}
& \Xi_{\text {hdet }}^{\ell} \circ \mu_{H}(h)=h_{A} h h_{A}^{-1} \text { in } H \\
& \mu_{A}(a)=h_{A} \rightarrow a \text { in } A \\
& =h_{A} a h_{A}^{-1} \text { in } \Lambda \text {. }
\end{aligned}
$$

Since hdet $=\epsilon$ and $\mu_{H}$ is given by $\bullet \mapsto h_{0} \bullet h_{0}^{-1}$, the first identity implies that $h_{0}^{-1} h_{A} \in Z\left(H^{\times}\right)$. Set $k_{A}=h_{0}^{-1} h_{A}$. Then, $k_{A} \in Z\left(H^{\times}\right)$and $k_{A}$ satisfies the identities $\mu_{A}(a)=\left(h_{0} k_{A}\right) \rightarrow a$ in $A$ and $\mu_{A}(a)=\left(h_{0} k_{A}\right) a\left(h_{0} k_{A}\right)^{-1}$ in $\Lambda$.

Conversely, assume that hdet $=\epsilon$ and that there exists $k_{A} \in Z\left(H^{\times}\right)$such that the identities $\mu_{A}(a)=\left(h_{0} k_{A}\right) \rightarrow a$ and $\mu_{A}(a)=\left(h_{0} k_{A}\right) a\left(h_{0} k_{A}\right)^{-1}$ hold in $A$ and $\Lambda$, respectively. Then, the Nakayama automorphism of $\Lambda$ given in 7.3.1 is inner (and associated with $\left.h_{0} k_{A} \in \Lambda^{\times}\right)$. Therefore, $\Lambda$ is Calabi-Yau. 
7.4.3. The previous result simplifies as follows when $A$ is assumed to be Calabi-Yau (see [16, 26, 37]).

Corollary. Let $H$ be a Calabi-Yau Hopf algebra. Let $A$ be a connected graded Hmodule algebra which is moreover Calabi-Yau. The following assertions are equivalent

(i) $A \sharp H$ is Calabi-Yau,

(ii) hdet $=\epsilon$.

Proof. Since $A$ is connected graded and Calabi-Yau, then $\mu_{A}=\operatorname{Id}_{A}$. The conclusion therefore follows from 7.4 .2

7.4.4. Combining the theorem in 7.4.2 and [14, Theorem 1.1] yields a proof of [30, Conjecture 6.4] for connected graded Artin-Schelter regular algebras.

Corollary. Let $A$ be an augmented skew Calabi-Yau algebra with Nakayama automorphism $\mu_{A}$. Then,

(1) $p \circ \operatorname{whdet}\left(\mu_{A}\right)=1$;

(2) if $A$ is connected graded and $\mu_{A}$ is also graded, then $\operatorname{hdet}\left(\mu_{A}\right)=1$.

Proof. Let $H=\mathbb{k} \mathbb{Z}$. It is Calabi-Yau and $\mu_{A}$ determines a structure of $H$-module augmented algebra on $A$. According to [14, Theorem 1.1], $A \sharp H$ is Calabi-Yau. Therefore, (1) follows from 7.4.1 (note that $\int_{\ell}=\epsilon$ according to 3.5.3), whereas (2) follows from 7.4 .2 .

\section{EXAmple: ACtions of $\mathcal{U}_{q}\left(\mathfrak{s l}_{2}\right)$ ON The QUANTUM PLANE}

Let $q \in \mathbb{C}^{\times}$. Assume that $q$ is not a root of unity. Let $A$ be the quantum plane $\mathbb{C}_{q}[x, y]$. Let $H$ be the quantum enveloping algebra $\mathcal{U}_{q}\left(\mathfrak{s l}_{2}\right)$. Assume that $\mathbb{C}_{q}[x, y]$ is endowed with a structure of $\mathcal{U}_{q}\left(\mathfrak{s l}_{2}\right)$-module algebra. This section applies Section 7.3 to the computation of a Nakayama automorphism of $\mathbb{C}_{q}[x, y] \sharp \mathcal{U}_{q}\left(\mathfrak{s l}_{2}\right)$. For this purpose, Section 8.1 recalls known material on $A$ and $H$, Section 8.2 endows the Koszul resolution of $A$ as a bimodule over itself with an action of $H$ so that it lies in $\mathcal{C}\left(\Delta_{0}\right)$, Section 8.3 computes the resulting action of $H$ on $\operatorname{Ext}_{A^{e}}^{2}\left(A, A^{e}\right)$ in terms of the mapping $\lambda$ introduced in 7.2.1. Section 8.4 computes the weak homological determinant of the action of $H$ on $A$, and Section 8.5 computes the Nakayama automorphism. The computations are made according to the classification of the actions of $H$ on $A$ made in [10].

8.1. Reminder on $\mathcal{U}_{q}\left(\mathfrak{s l}_{2}\right)$ and on $\mathbb{C}_{q}[x, y]$. As a $\mathbb{C}$-algebra, $\mathcal{U}_{q}\left(\mathfrak{s l}_{2}\right)$ is given by generators $E, F, K, K^{-1}$ and relations

$$
K K^{-1}=1=K^{-1} K, K E K^{-1}=q^{2} E, K F K^{-1}=q^{-2} F,[E, F]=\frac{K-K^{-1}}{q-q^{-1}} .
$$

The comultiplication, the counit and the antipode of $\mathcal{U}_{q}\left(\mathfrak{s l}_{2}\right)$ are given by

$$
\begin{gathered}
\Delta(K)=K \otimes K, \Delta(E)=E \otimes K+1 \otimes E, \Delta(F)=F \otimes 1+K^{-1} \otimes F, \\
\epsilon(K)=1, \epsilon(E)=0, \epsilon(F)=0, \\
S(K)=K^{-1}, S(E)=-E K^{-1}, S(F)=-K F .
\end{gathered}
$$

$H$ is 2-Calabi-Yau (see [7, Theorem 3.3.2]), and hence (see 3.5.3 and its dual version)

$$
\int_{\ell}=\int_{r}=\epsilon
$$

The quantum plane is the Koszul $\mathbb{C}$-algebra $A=\mathbb{C}_{q}[x, y]=\mathbb{C}\langle x, y \mid y x=q x y\rangle$. Let $V=\mathbb{C} \cdot x \oplus \mathbb{C} \cdot y$. Then, $\mathbb{C}_{q}[x, y]$ admits the following Koszul resolution as a bimodule over itself ([35, Proposition 4.1])

$$
P: \quad 0 \rightarrow A \otimes \Lambda^{2} V \otimes A \stackrel{d}{\rightarrow} A \otimes V \otimes A \stackrel{d}{\rightarrow} A \otimes A \rightarrow 0
$$


where, using "|" instead of " $\otimes$ " for the ease of readability,

$$
\begin{aligned}
d(1|x| 1) & =x|1-1| x \\
d(1|y| 1) & =y|1-1| y \\
d(1|x \wedge y| 1) & =x|y| 1-q^{-1}|y| x-q^{-1} y|x| 1+1|x| y .
\end{aligned}
$$

The algebra $\mathbb{C}_{q}[x, y]$ is (connected $\mathbb{N}$-graded) Artin-Schelter regular (see [3, p. 172]), and hence skew Calabi-Yau (see [30, Lemma 1.2]) with a Nakayama automorphism as follows (see [29, p. 76]). Let $\varphi_{A} \in \operatorname{Hom}_{A^{e}}\left(A \otimes \Lambda^{2} V \otimes A, A^{e}\right)$ be the following 2-cocycle whose cohomology class in $\operatorname{Ext}_{A^{e}}^{2}\left(A, A^{e}\right)$ is denoted by $e_{A}$

$$
\begin{aligned}
\varphi_{A}: \quad A \otimes \Lambda^{2} V \otimes A & \rightarrow A \otimes A \\
1|x \wedge y| 1 & \mapsto 1 \otimes 1 .
\end{aligned}
$$

Then, $e_{A}$ is a free generator of the left $A$-module $\operatorname{Ext}_{A^{e}}^{2}\left(A, A^{e}\right)$. By considering the coboundaries of the two 1-cocycles $A \otimes V \otimes A \rightarrow A \otimes A$ defined by

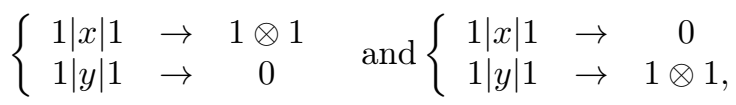

respectively, it appears that $e_{A} x=q^{-1} x e_{A}$ and $e_{A} y=q y e_{A}$. Therefore, the Nakayama automorphism $\mu_{A}$ of $A$ corresponding to $e_{A}$ is given by

$$
\mu_{A}(x)=q^{-1} x \text { and } \mu_{A}(y)=q y .
$$

\begin{tabular}{|c|c|c|c|}
\hline case & actions of $E, F, K$ on $x, y$ & & parameters \\
\hline 0 & $\begin{array}{l}K \rightarrow x= \pm x \\
E \rightarrow x=0 \\
F \rightarrow x=0\end{array}$ & $\begin{array}{l}K \rightarrow y= \pm y \\
E \rightarrow y=0 \\
F \rightarrow y=0\end{array}$ & $\emptyset$ \\
\hline 1 & $\begin{array}{l}K \rightarrow x=q x \\
E \rightarrow x=0 \\
F \rightarrow x=b_{0}^{-1} x y\end{array}$ & $\begin{array}{l}K \rightarrow y=q^{-2} y \\
E \rightarrow y=b_{0} \\
F \rightarrow y=-q b_{0}^{-1} y^{2}\end{array}$ & $b_{0} \in \mathbb{C}^{\times}$ \\
\hline 2 & $\begin{array}{l}K \rightarrow x=q^{2} x \\
E \rightarrow x=-q c_{0}^{-1} x^{2} \\
F \rightarrow x=c_{0}\end{array}$ & $\begin{array}{l}K \rightarrow y=q^{-1} y \\
E \rightarrow y=c_{0}^{-1} x y \\
F \rightarrow y=0\end{array}$ & $c_{0} \in \mathbb{C}^{\times}$ \\
\hline 3 & $\begin{array}{l}K \rightarrow x=q^{-2} x \\
E \rightarrow x=a_{0} \\
F \rightarrow x=-q a_{0}^{-1} x^{2}+t y^{4}\end{array}$ & $\begin{array}{l}K \rightarrow y=q^{-1} y \\
E \rightarrow y=0 \\
F \rightarrow y=-q a_{0}^{-1} x y+s y^{3}\end{array}$ & $\begin{array}{l}a_{0} \in \mathbb{C}^{\times} \\
s, t \in \mathbb{C}\end{array}$ \\
\hline 4 & $\begin{array}{l}K \rightarrow x=q x \\
E \rightarrow x=-q d_{0}^{-1} x y+s x^{3} \\
F \rightarrow x=0\end{array}$ & $\begin{array}{l}K \rightarrow y=q^{2} y \\
E \rightarrow y=-q d_{0}^{-1} y^{2}+t x^{4} \\
F \rightarrow y=d_{0}\end{array}$ & $\begin{array}{l}d_{0} \in \mathbb{C}^{\times} \\
s, t \in \mathbb{C}\end{array}$ \\
\hline 5 & $\begin{array}{l}K \rightarrow x=q x \\
E \rightarrow x=0 \\
F \rightarrow x=\tau^{-1} y\end{array}$ & $\begin{array}{l}K \rightarrow y=q^{-1} y \\
E \rightarrow y=\tau x \\
F \rightarrow y=0\end{array}$ & $\tau \in \mathbb{C}^{\times}$ \\
\hline
\end{tabular}

The actions of $\mathcal{U}_{q}\left(\mathfrak{s l}_{2}\right)$ on $\mathbb{C}_{q}[x, y]$ are classified into six families with parameters (see [10, Table 1] and Table 1 below). The figures in the first column of Table 1 serve as an internal reference for the corresponding action. Except for the first and last case, the action does not preserve the augmentation ideal of $\mathbb{C}_{q}[x, y]$. Therefore $\mathbb{C}_{q}[x, y] \sharp \mathcal{U}_{q}\left(\mathfrak{s l}_{2}\right)$ may be non augmented and non connected graded.

TABLE 1. Classification of the actions of $\mathcal{U}_{q}\left(\mathfrak{s l}_{2}\right)$ on $\mathbb{C}_{q}[x, y]$

\subsection{Action of $\mathcal{U}_{q}\left(\mathfrak{s l}_{2}\right)$ on the Koszul resolution $\mathbb{C}_{q}[x, y]$.}

Lemma. There exists an action of $\mathcal{U}_{q}\left(\mathfrak{s l}_{2}\right)$ on the Koszul resolution $P$ of $\mathbb{C}_{q}[x, y]$ such that

- $P$ is complex of $\mathcal{U}_{q}\left(\mathfrak{s l}_{2}\right)$-equivariant $\mathbb{C}_{q}[x, y]$-bimodules (or, $P \in \mathcal{C}\left(\Delta_{0}\right)$ ),

- the action on $\mathbb{C}_{q}[x, y] \otimes \mathbb{C}_{q}[x, y]$ is the natural one $\left(h-(a \otimes b)=h_{1} \rightarrow\right.$ $\left.a \otimes h_{2} \rightarrow b\right)$, 
- if $t_{1}, t_{2} \in \mathbb{C}^{\times}$denote the scalars such that $K \rightarrow x=t_{1} x$ and $K \rightarrow y=t_{2} y$ (see Table 1), then

$$
\begin{aligned}
K \rightarrow 1|x| 1 & =t_{1}|x| 1 & K^{-1} \rightarrow 1|x| 1 & =t_{1}^{-1}|x| 1 \\
K \rightarrow 1|y| 1 & =t_{2}|y| 1 & K^{-1} \rightarrow 1|y| 1 & =t_{2}^{-1}|y| 1 \\
K \rightarrow 1|x \wedge y| 1 & =t_{1} t_{2}|x \wedge y| 1 & K^{-1} \rightarrow 1|x \wedge y| 1 & =\left(t_{1} t_{2}\right)^{-1}|x \wedge y| 1
\end{aligned}
$$

- the actions of $E$ and $F$ on $1|x| 1,1|y| 1$ and $1|x \wedge y| 1$ are such as in Table 2

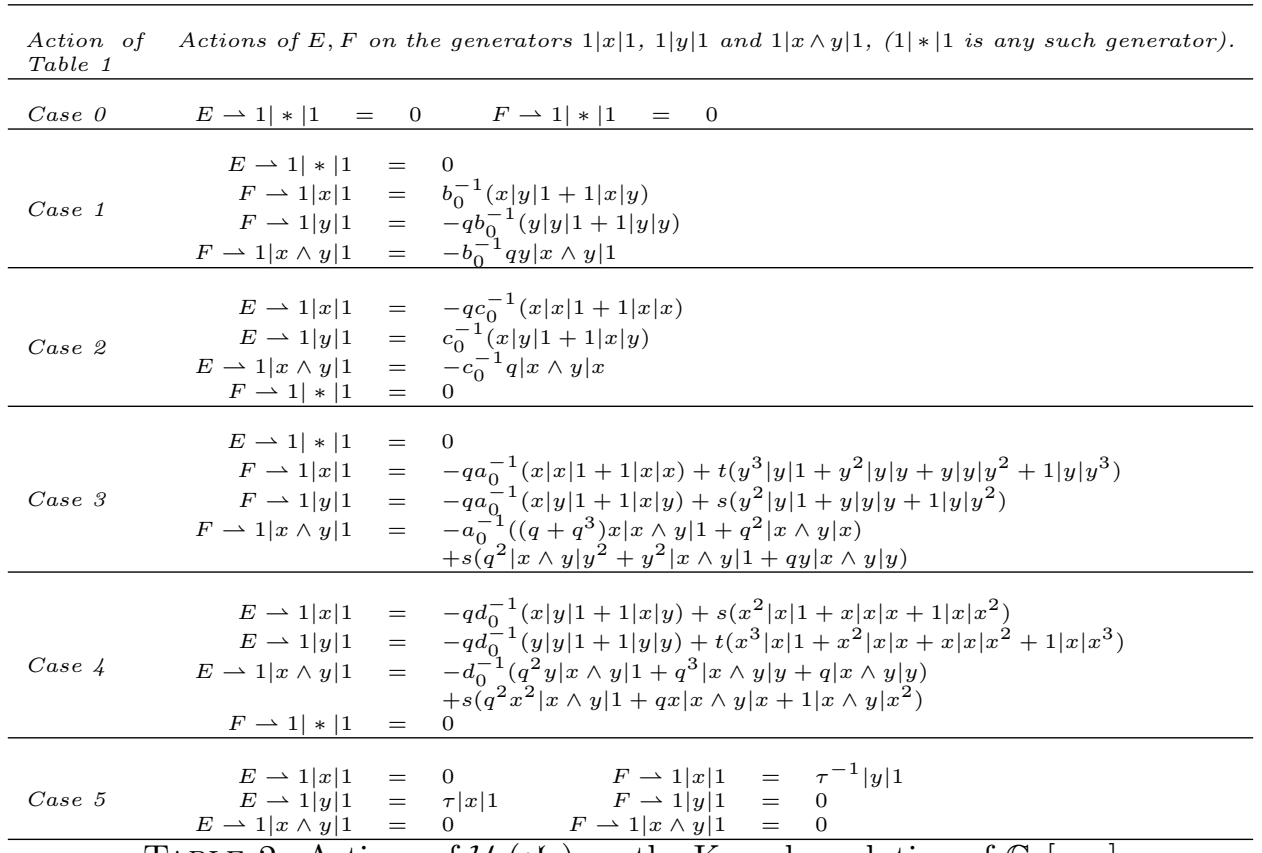

TABLE 2. Actions of $\mathcal{U}_{q}\left(\mathfrak{s l}_{2}\right)$ on the Koszul resolution of $\mathbb{C}_{q}[x, y]$

Proof. For each $h \in\left\{E, F, K, K^{-1}\right\}$, the action of $h$ on $\mathbb{C}_{q}[x, y] \otimes \mathbb{C}_{q}[x, y], 1|x| 1$, $1|y| 1$ and $1|x \wedge y| 1$ given in the statement of the lemma may be extended to a $\mathbb{C}$-linear mapping

$$
\begin{aligned}
P & \rightarrow P \\
u & \mapsto h \rightarrow u
\end{aligned}
$$

in such a way that, for all $g \in\{x, y, x \wedge y\}$, and $a, b$.

$$
h \rightarrow(a|g| b)=\left(h_{1} \rightarrow a\right)\left(h_{2} \rightarrow(1|g| 1)\right)\left(h_{3} \rightarrow b\right) .
$$

Elementary (though tedious) computations show that this is a morphism of complexes of vector spaces. In order to prove the lemma, it is therefore sufficient to prove that, for all $u \in\{1|x| 1,1|y| 1,1|x \wedge y| 1\}$,

(a) $K^{-1} \rightarrow(K \rightarrow u)=u=K \rightarrow\left(K^{-1} \rightarrow u\right)$,

(b) $K \rightarrow(E \rightarrow u)=q^{2} E \rightarrow(K \rightarrow u)$,

(c) $K \rightarrow(F \rightarrow u)=q^{-2} F \rightarrow(K \rightarrow u)$,

(d) $E \rightarrow(F \rightarrow u)-F \rightarrow(E \rightarrow u)=\frac{1}{q-q^{-1}}\left(K \rightarrow u-K^{-1} \rightarrow u\right)$.

The rest of the proof of the lemma explains why these equalities hold true.

Proof of (a). This follows from the definition of the actions of $K$ and $K^{-1}$.

Proof of (b) and (c). In case 0 , the equalities may be checked directly. In the other cases, denote by $\gamma$ the relative integer such that $K \rightarrow u=q^{\gamma} u$. Then, it can be checked from Table 2 that $E \rightarrow u($ or $F \rightarrow u)$ is either 0 or an eigenvector of 
the action of $K$ with eigenvalue $q^{\gamma+2}$ (or $q^{\gamma-2}$ ), which proves the equality (b) (or, (c), respectively).

Proof of $(d)$. In case 0 , the equality may be checked directly from Table 2

Assume that the considered action is the one of case 1 . Then, using that $E \rightarrow$ $x=E \rightarrow 1|*| 1=0$,

$$
\begin{aligned}
E \rightarrow(F \rightarrow(1|x| 1)) & =b_{0}^{-1} E \rightarrow(x|y| 1+1|x| y) \\
& =b_{0}^{-1}(1|x| 1)(E \rightarrow y) \\
& =1|x| 1 \\
F \rightarrow(E \rightarrow(1|x| 1)) & =0 \\
K \rightarrow(1|x| 1) & =q|x| 1 \\
K^{-1} \rightarrow(1|x| 1) & =q^{-1}|x| 1,
\end{aligned}
$$

from which the considered equality may be checked directly when $u=1|x| 1$. Next,

$$
\begin{aligned}
E \rightarrow(F \rightarrow(1|y| 1)) & =-q b_{0}^{-1} E \rightarrow(y|y| 1+1|y| y) \\
& =-q b_{0}^{-1}((E \rightarrow y)(K \rightarrow(1|y| 1))+(1|y| 1)(E \rightarrow y)) \\
& =-\left(q^{-1}+q\right)|y| 1 \\
F \rightarrow(E \rightarrow(1|y| 1)) & =0 \\
K \rightarrow(1|y| 1) & =q^{-2}|y| 1 \\
K^{-1} \rightarrow(1|y| 1) & =q^{2}|y| 1
\end{aligned}
$$

from which the considered equality may be proved directly when $u=1|y| 1$. Finally,

$$
\begin{aligned}
E \rightarrow(F \rightarrow(1|x \wedge y| 1)) & =-b_{0}^{-1} q E \rightarrow(y|x \wedge y| 1) \\
& =-b_{0}^{-1} q(E \rightarrow y)(K \rightarrow(1|x \wedge y| 1) \\
& =-1|x \wedge y| 1 \\
F \rightarrow(E \rightarrow(1|x \wedge y| 1)) & =0 \\
K \rightarrow(1|x \wedge y| 1) & =q^{-1}|x \wedge y| 1 \\
K^{-1} \rightarrow(1|x \wedge y| 1) & =q|x \wedge y| 1,
\end{aligned}
$$

from which the considered equality may be proved directly when $u=1|x \wedge y| 1$.

Now, assume that the considered action is the one of case 2. Note that, there is an isomorphism of Hopf algebras between $\mathcal{U}_{q}\left(\mathfrak{s l}_{2}\right)$ and $\mathcal{U}_{q}\left(\mathfrak{s l}_{2}\right)^{\text {op }}$ which exchanges $K$ and $K^{-1}$ and exchanges $E$ and $F$. Under this isomorphism, the action of case 2 corresponds to the one of case 1 provided that the following changes are made,

- exchange $x$ and $y$, and next,

- transform each tensor $a|*| b$ into $b|*| a$.

The previous considerations for case 1 therefore apply to case 2 , and hence prove the equality in the latter case.

Now assume that the considered action is the one of case 3 . Then, using that $E \rightarrow y=E \rightarrow(1|*| 1)=0$,

$$
\begin{aligned}
E \rightarrow(F \rightarrow(1|x| 1))= & -q a_{0}^{-1} E \rightarrow(x|x| 1+1|x| x) \\
& +t E \rightarrow\left(y^{3}|y| 1+\cdots\right) \\
= & -q a_{0}^{-1}((E \rightarrow x)(K \rightarrow(1|x| 1))+(1|x| 1)(E \rightarrow x)) \\
= & -\left(q+q^{-1}\right)|x| 1 \\
F \rightarrow(E \rightarrow(1|x| 1))= & 0 \\
K \rightarrow(1|x| 1)= & q^{-2}|x| 1 \\
K^{-1} \rightarrow(1|x| 1)= & q^{2}|x| 1,
\end{aligned}
$$


from which the considered equality may be checked directly when $u=1|x| 1$. Next,

$$
\begin{aligned}
E \rightarrow(F \rightarrow(1|y| 1))= & -q a_{0}^{-1} E \rightarrow(x|y| 1+1|x| y) \\
& +s E \rightarrow\left(y^{2}|y| 1+\cdots\right) \\
= & -q a_{0}^{-1}(E \rightarrow x)(K \rightarrow(1|y| 1)) \\
= & -1|y| 1 \\
F \rightarrow(E \rightarrow(1|y| 1))= & 0 \\
K \rightarrow(1|y| 1)= & q^{-1}|y| 1 \\
K^{-1} \rightarrow(1|y| 1)= & q|y| 1,
\end{aligned}
$$

from which the considered equality may be proved directly when $u=1|y| 1$. Finally,

$$
\begin{aligned}
E \rightarrow(F \rightarrow(1|x \wedge y| 1))= & -a_{0}^{-1} E \rightarrow\left(\left(q+q^{3}\right) x|x \wedge y| 1+q^{2}|x \wedge y| x\right) \\
& +s E \rightarrow\left(q^{2}|x \wedge y| y^{2}+\cdots\right) \\
= & -a_{0}^{-1}\left(\left(q+q^{3}\right)(E \rightarrow x)(K \rightarrow(1|x \wedge y| 1))\right. \\
& \left.+\left(q^{2}|x \wedge y| 1\right)(E \rightarrow x)\right) \\
= & -\left(q^{-2}+1+q^{2}\right)|x \wedge y| 1 \\
F \rightarrow(E \rightarrow(1|x \wedge y| 1))= & 0 \\
K \rightarrow(1|x \wedge y| 1)= & q^{-3}|x \wedge y| 1 \\
K^{-1} \rightarrow(1|x \wedge y| 1)= & q^{3}|x \wedge y| 1,
\end{aligned}
$$

from which the considered equality may be proved directly when $u=1|x \wedge y| 1$.

The equality in case 4 may be deduced from the one in case 3 the same way the one in case 2 was deduced from the one in case 1.

To end with, when the considered action is the one of case 5 , then the equality may be checked directly from Table 2

8.3. The action of $\mathcal{U}_{q}\left(\mathfrak{s l}_{2}\right)$ on $\operatorname{Ext}_{\mathbb{C}_{q}[x, y]^{e}}^{2}\left(\mathbb{C}_{q}[x, y], \mathbb{C}_{q}[x, y]^{e}\right)$.

Lemma. Let $e_{A}$ be the free generator of $\operatorname{Ext}_{\mathbb{C}_{q}[x, y]^{e}}^{2}\left(\mathbb{C}_{q}[x, y], \mathbb{C}_{q}[x, y]^{e}\right)$ introduced in 8.1.0.3. Denote by $\lambda$ the mapping $\mathcal{U}_{q}\left(\mathfrak{s l}_{2}\right) \rightarrow \mathbb{C}_{q}[x, y]$ such that the identity $h \rightarrow e_{A}=\lambda(h) e_{A}$ holds. Then, Table 3 describes the mapping $\lambda$.

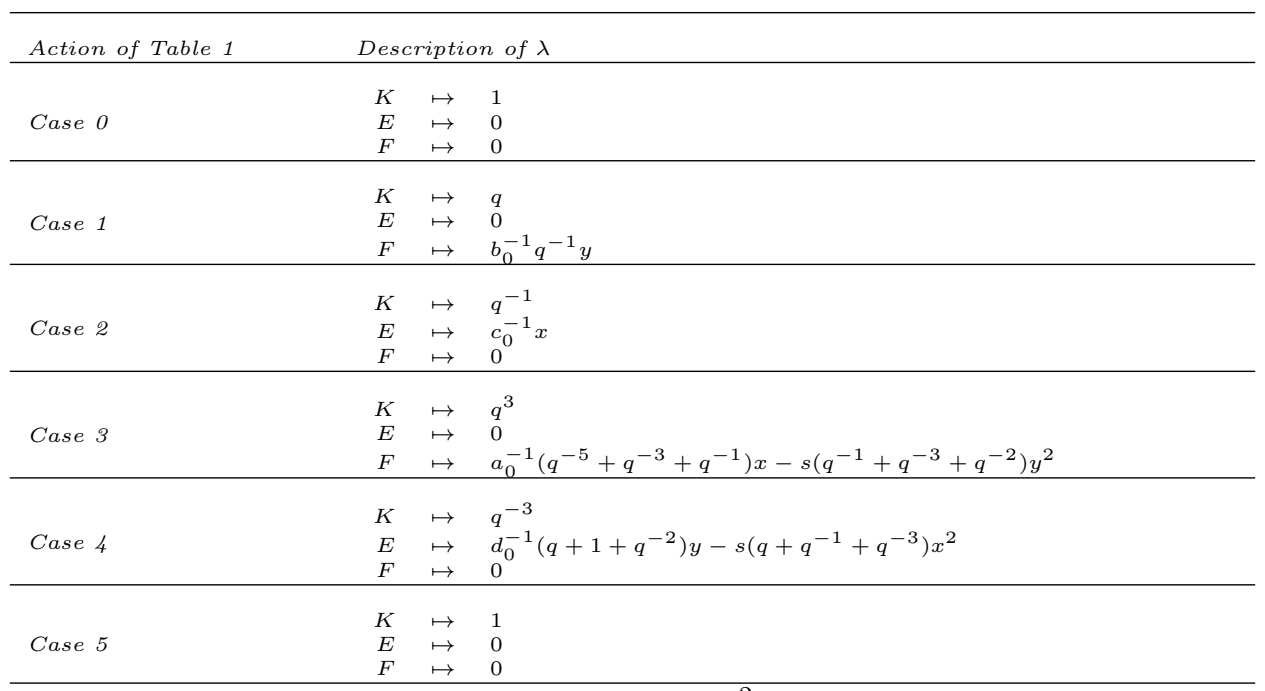

TABLE 3. The action of $\mathcal{U}_{q}\left(\mathfrak{s l}_{2}\right)$ on $\operatorname{Ext}_{\mathbb{C}_{q}[x, y]}^{2}\left(\mathbb{C}_{q}[x, y], \mathbb{C}_{q}[x, y]^{e}\right)$

Proof. Let $\varphi_{A}$ be as in 8.1.0.3). For all $h$, then $h \rightarrow e_{A}$ is the cohomology class of $h \rightarrow \varphi_{A}$, which is given by 5.4.0.1; In view of the considerations of 8.1. if $\sum_{i} a_{i} \otimes b_{i}$ is an element of $\mathbb{C}_{q}[x, y]^{e}$ such that $h \rightarrow \varphi_{A}(1|x \wedge y| 1)=\sum_{i} a_{i} \otimes b_{i}$, then 
$h \rightarrow \varphi_{A}$ equals $\sum_{i} b_{i} \varphi_{A} a_{i}$, which is cohomologous to $\left(\sum_{i} b_{i} \mu_{A}\left(a_{i}\right)\right) \varphi_{A}$, and hence $\lambda(h)=\sum_{i} b_{i} \mu_{A}\left(a_{i}\right)$. In order to determine $\lambda(K), \lambda(E)$ and $\lambda(F)$, it is therefore enough to determine $\left(h \rightarrow \varphi_{A}\right)(1|x \wedge y| 1)$ for $h \in\{K, E, F\}$. For this purpose, the following piece of notation is used for all $h, k, a, b$,

$$
(h \otimes k) \rightarrow(a \otimes b)=(h \rightarrow a) \otimes(k \rightarrow b) .
$$

First consider $\lambda(K)$. Using (5.4.0.1), and using the fact that $K^{-1} \rightarrow(1|x \wedge y| 1) \in$ $\mathbb{C} \cdot(1|x \wedge y| 1)$, then

$$
\begin{aligned}
\left(K \rightarrow \varphi_{A}\right)(1|x \wedge y| 1) & =(K \otimes K) \rightarrow \varphi_{A}\left(K^{-1} \rightarrow(1|x \wedge y| 1)\right) \\
& =\varphi_{A}\left(K^{-1} \rightarrow(1|x \wedge y| 1)\right) .
\end{aligned}
$$

Therefore, if $t_{1}, t_{2} \in \mathbb{C}^{\times}$denote the scalars such that $K \rightarrow x=t_{1} x$ and $K \rightarrow$ $y=t_{2} y$, respectively, then $\left(K^{-1} \rightarrow \varphi_{A}\right)(1|x \wedge y| 1)=\left(t_{1} t_{2}\right)^{-1}|x \wedge y| 1$, and hence $\lambda(K)=\left(t_{1} t_{2}\right)^{-1}$. Whence the values of $\lambda(K)$ given in Table 3 .

Next, consider $\lambda(E)$. Using 5.4.0.1 and the equality $E \rightarrow 1=0$, and using that $K^{-1} \rightarrow(1|x \wedge y| 1) \in \mathbb{C} \cdot(1|x \wedge y| 1)$, then

$$
\begin{aligned}
\left(E \rightarrow \varphi_{A}\right)(1|x \wedge y| 1)= & \left(q^{2} E \otimes 1\right) \rightarrow \varphi_{A}(1|x \wedge y| 1) \\
& -(K \otimes 1) \rightarrow \varphi_{A}\left(\left(E K^{-1}\right) \rightarrow(1|x \wedge y| 1)\right) \\
& +(K \otimes E) \rightarrow \varphi_{A}\left(K^{-1} \rightarrow(1|x \wedge y| 1)\right) \\
= & -(K \otimes 1) \rightarrow \varphi_{A}\left(\left(E K^{-1}\right) \rightarrow(1|x \wedge y| 1)\right) .
\end{aligned}
$$

Now, use Table 2 in order to determine $\left(E \rightarrow \varphi_{A}\right)(1|x \wedge y| 1)$. This is zero in cases $0,1,3$ and 5 ; Therefore, $\lambda(E)=0$ in these cases. In case 2 , then $\left(E K^{-1}\right) \rightarrow$ $(1|x \wedge y| 1)=-c_{0}^{-1}|x \wedge y| x$, and hence $\left(E \rightarrow \varphi_{A}\right)(1|x \wedge y| 1)=c_{0}^{-1} \otimes x ;$ Accordingly, $\lambda(E)=c_{0}^{-1} x$. And in case 4 , then $\left(E K^{-1}\right) \rightarrow(1|x \wedge y| 1)$ equals $q^{-3}\left(-d_{0}^{-1}\left(q^{2} y|x \wedge y| 1+q^{3}|x \wedge y| y+q|x \wedge y| y\right)+s\left(q^{2} x^{2}|x \wedge y| 1+q x|x \wedge y| x+1|x \wedge y| x^{2}\right)\right)$, and hence $\left(E \rightarrow \varphi_{A}\right)(1|x \wedge y| 1)$ equals

$$
q^{-3}\left(d_{0}^{-1}\left(q^{4} y \otimes 1+q^{3} \otimes y+q \otimes y\right)-s\left(q^{4} x^{2} \otimes 1+q^{2} x \otimes x+1 \otimes x^{2}\right)\right) ;
$$

Consequently

$$
\lambda(E)=d_{0}^{-1}\left(q+1+q^{-2}\right) y-s\left(q+q^{-1}+q^{-3}\right) x^{2} .
$$

Finally, consider $\lambda(F)$. Then,

$$
\begin{aligned}
\left(F \rightarrow \varphi_{A}\right)(1|x \wedge y| 1)= & (1 \otimes F) \rightarrow \varphi_{A}(1|x \wedge y| 1) \\
& -\left(1 \otimes K^{-1}\right) \rightarrow \varphi_{A}((K F) \rightarrow(1|x \wedge y| 1)) \\
& +\left(q^{-2} F \otimes K^{-1}\right) \rightarrow \varphi_{A}(K \rightarrow(1|x \wedge y| 1)) \\
= & -\left(1 \otimes K^{-1}\right) \rightarrow \varphi_{A}((K F) \rightarrow(1|x \wedge y| 1)),
\end{aligned}
$$

from which the value of $\lambda(F)$ is determined using considerations analogous to those used in order to determine $\lambda(E)$.

\subsection{Computation of the weak homological determinant.}

Lemma. Let $e_{A}$ be the free generator of $\operatorname{Ext}_{\mathbb{C}_{q}[x, y]^{e}}^{2}\left(\mathbb{C}_{q}[x, y], \mathbb{C}_{q}[x, y]^{e}\right)$ introduced in 8.1.0.3. Denote by whdet the weak homological determinant corresponding to $e_{A}$. Then, Table 4 gives the values of whdet on $K, E$ and $F$. And Table 5 gives the values of $\theta_{\text {whdet }}$ on $K, E$ and $F$.

Proof. Let $\lambda$ be such as in 8.3 . Recall from 7.2.1 and 7.2.2 that

- $\lambda$ is characterised by the identity $h \rightarrow e_{A}=\lambda(h) e_{A}$ and it satisfies the identity $\lambda(h k)=h_{1} \rightarrow \lambda(k) \lambda\left(h_{2}\right)$,

- whdet is defined by whdet $(h)=S^{-2}\left(h_{2}\right) \rightarrow \lambda\left(S^{-3}\left(h_{1}\right)\right)$,

- $\theta_{\text {whdet }}$ is defined by $\theta_{\text {whdet }}(h)=\operatorname{whdet}\left(S^{2}\left(h_{1}\right)\right) h_{2}$.

Before proving the lemma, it is convenient to prove the following equalities first, 


\begin{tabular}{|c|c|c|c|}
\hline Action of Table 1 & Desc & ipti & $n$ of whdet \\
\hline Case $O$ & $\begin{array}{l}K \\
E \\
F \\
\end{array}$ & $\begin{array}{l}\mapsto \\
\mapsto \\
\mapsto\end{array}$ & $\begin{array}{l}1 \\
0 \\
0 \\
\end{array}$ \\
\hline Case 1 & $\begin{array}{l}K \\
E \\
F\end{array}$ & $\begin{array}{l}\mapsto \\
\mapsto \\
\mapsto\end{array}$ & $\begin{array}{l}q^{-1} \\
0 \\
-q^{2} b_{0}^{-1} y\end{array}$ \\
\hline Case 2 & $\begin{array}{l}K \\
E \\
F \\
\end{array}$ & $\begin{array}{l}\mapsto \\
\mapsto \\
\mapsto\end{array}$ & $\begin{array}{l}q \\
-q^{-1} c_{0}^{-1} x \\
0\end{array}$ \\
\hline Case 3 & $\begin{array}{l}K \\
E \\
F \\
\end{array}$ & $\begin{array}{l}\mapsto \\
\mapsto \\
\mapsto\end{array}$ & $\begin{array}{l}q^{-3} \\
0 \\
s\left(1+q^{2}+q^{4}\right) y^{2}-a_{0}^{-1}\left(q+q^{3}+q^{4}\right) x\end{array}$ \\
\hline Case 4 & $\begin{array}{l}K \\
E \\
F\end{array}$ & $\begin{array}{l}\mapsto \\
\mapsto \\
\mapsto\end{array}$ & $\begin{array}{l}q^{3} \\
s\left(q^{2}+1+q^{-2}\right) x^{2}-d_{0}^{-1}\left(q^{2}+q+q^{-1}\right) y \\
0\end{array}$ \\
\hline Case 5 & $\begin{array}{l}K \\
E \\
F\end{array}$ & $\begin{array}{l}\mapsto \\
\mapsto \\
\mapsto\end{array}$ & $\begin{array}{l}1 \\
0 \\
0\end{array}$ \\
\hline
\end{tabular}

TABLE 4. The values of whdet on $K, E$ and $F$

\begin{tabular}{|c|c|c|}
\hline Action of Table 1 & Descripti & $n$ of $\theta_{\text {whdet }}$ \\
\hline Case $O$ & $\begin{array}{ll}K & \mapsto \\
E & \mapsto \\
F & \mapsto\end{array}$ & $\begin{array}{l}K \\
E \\
F\end{array}$ \\
\hline Case 1 & $\begin{aligned} K & \mapsto \\
E & \mapsto \\
F & \mapsto\end{aligned}$ & $\begin{array}{l}q^{-1} K \\
E \\
q F-b_{0}^{-1} y K\end{array}$ \\
\hline Case 2 & $\begin{aligned} K & \mapsto \\
E & \mapsto \\
F & \mapsto\end{aligned}$ & $\begin{array}{l}q K \\
E-q c_{0}^{-1} x K \\
q^{-1} F\end{array}$ \\
\hline Case 3 & $\begin{aligned} K & \mapsto \\
E & \mapsto \\
F & \mapsto\end{aligned}$ & $\begin{array}{l}q^{-3} K \\
E \\
q^{3} F+s\left(q^{-2}+1+q^{2}\right) y^{2}-a_{0}^{-1}\left(q^{-1}+q+q^{2}\right) x\end{array}$ \\
\hline Case 4 & $\begin{aligned} K & \mapsto \\
E & \mapsto \\
F & \mapsto\end{aligned}$ & $\begin{array}{l}q^{3} K \\
E+s\left(q^{4}+q^{2}+1\right) x^{2} K-d_{0}^{-1}\left(q^{4}+q^{3}+q\right) y K \\
q^{-3} F\end{array}$ \\
\hline Case 5 & $\begin{array}{ll}K & \mapsto \\
E & \mapsto \\
F & \mapsto\end{array}$ & $\begin{array}{l}K \\
E \\
F\end{array}$ \\
\hline
\end{tabular}

TABLE 5. The values of $\theta_{\text {whdet }}$ on $K, E$ and $F$

(1) $\operatorname{whdet}(K)=\lambda\left(K^{-1}\right)$ and $\theta_{\text {whdet }}(K)=\lambda\left(K^{-1}\right) K$,

(2) $\operatorname{whdet}\left(K^{-1}\right)=\lambda(K)$ and $\theta_{\text {whdet }}\left(K^{-1}\right)=\lambda(K) K^{-1}$,

(3) $\operatorname{whdet}(E)=-q^{-2} \lambda(E) \lambda\left(K^{-1}\right)$ and $\theta_{\text {whdet }}(E)=E-\lambda(E) \lambda\left(K^{-1}\right) K$,

(4) $\operatorname{whdet}(F)=-q^{2} \lambda(K) \lambda(F)$ and $\theta_{\text {whdet }}(F)=\lambda(K) F-\lambda(K) \lambda(F)$.

(1) Since $\lambda(1)=1$ and $\lambda(K) \in \mathbb{C}$, then $\lambda\left(K^{-1}\right)=\lambda(K)^{-1}$ and $\lambda\left(K^{-1}\right) \in$ C. Therefore, whdet $(K)=K \rightarrow \lambda\left(K^{-1}\right)=\lambda\left(K^{-1}\right)$, and hence $\theta_{\text {whdet }}(K)=$ whdet $(K) K=\lambda\left(K^{-1}\right) K$.

(2) is proved using the same considerations.

(3) $\operatorname{whdet}(E)=S^{-2}(E) \rightarrow \lambda(1)+K \rightarrow \lambda\left(S^{-3}(E)\right)$. Note that $S^{-2}(E)=q^{-2} E$; Moreover, since $S(E)=-E K^{-1}$ and $S(K)=K^{-1}$, then $S^{-1}(E)=-K^{-1} E$ and 
$S^{-3}(E)=-q^{-2} K^{-1} E$. Therefore,

$$
\begin{array}{rlr}
\operatorname{whdet}(E) & =q^{-2} E \rightarrow 1-q^{-2} K \rightarrow \lambda\left(K^{-1} E\right) & \\
& =-q^{-2} K \rightarrow\left(K^{-1} \rightarrow \lambda(E) \lambda\left(K^{-1}\right)\right) & \\
& =-q^{-2} \lambda(E) \lambda\left(K^{-1}\right) & \left(\text { since } \lambda\left(K^{-1}\right) \in \mathbb{C}\right) .
\end{array}
$$

Now,

$$
\begin{aligned}
\theta_{\text {whdet }}(E) & =\operatorname{whdet}\left(S^{2}(E)\right) K+\operatorname{whdet}(1) E \\
& =-\lambda(E) \lambda\left(K^{-1}\right) K+E
\end{aligned}
$$

$$
\begin{aligned}
\operatorname{whdet}(F) & =S^{-2}(1) \rightarrow \lambda\left(S^{-3}(F)\right)+S^{-2}(F) \rightarrow \lambda\left(S^{-3}\left(K^{-1}\right)\right) \\
& =q^{2} \lambda\left(S^{-1}(F)\right)+q^{2} F \rightarrow \lambda(K) \\
& =q^{2} \lambda\left(S^{-1}(F)\right)
\end{aligned}
$$$$
\text { (since } \lambda(K) \in \mathbb{C}) \text {. }
$$

Since $S(F)=-K F$ and $S(K)=K^{-1}$, then $S^{-1}(F)=-F K$. Therefore,

$$
\begin{aligned}
\operatorname{whdet}(F) & =-q^{2} \lambda(F K) \\
& =-q^{2}\left(F \rightarrow \lambda(K) \lambda(1)+K^{-1} \rightarrow \lambda(K) \lambda(F)\right) \\
& =-q^{2} \lambda(K) \lambda(F)
\end{aligned}
$$

(since $\lambda(K), \lambda(1) \in \mathbb{C})$.

Now,

$$
\begin{aligned}
\theta_{\text {whdet }}(F) & =\text { whdet }\left(S^{2}(F)\right)+\operatorname{whdet}\left(S^{2}\left(K^{-1}\right)\right) F \\
& =q^{-2} \operatorname{whdet}(F)+\lambda(K) F \\
& =-\lambda(K) \lambda(F)+\lambda(K) F .
\end{aligned}
$$

Now, using equalities (1), (2), (3), and (4), it is possible to recover Table 4 and Table 5 using Table 3 , which proves the lemma.

\subsection{Description of the Nakayama automorphism.}

Proposition. Let $q \in \mathbb{C}^{\times}$be a non root of unity. Assume that $\mathbb{C}_{q}[x, y]$ is endowed with a structure of $\mathcal{U}_{q}\left(\mathfrak{s l}_{2}\right)$-module algebra. Then, $\mathbb{C}_{q}[x, y] \sharp \mathcal{U}_{q}\left(\mathfrak{s l}_{2}\right)$ is a skew CalabiYau algebra with a Nakayama automorphism given by

$$
x \mapsto q^{-1} x \text { and } y \mapsto q y
$$

and which values on $K, E$ and $F$ are given in Table 6 .

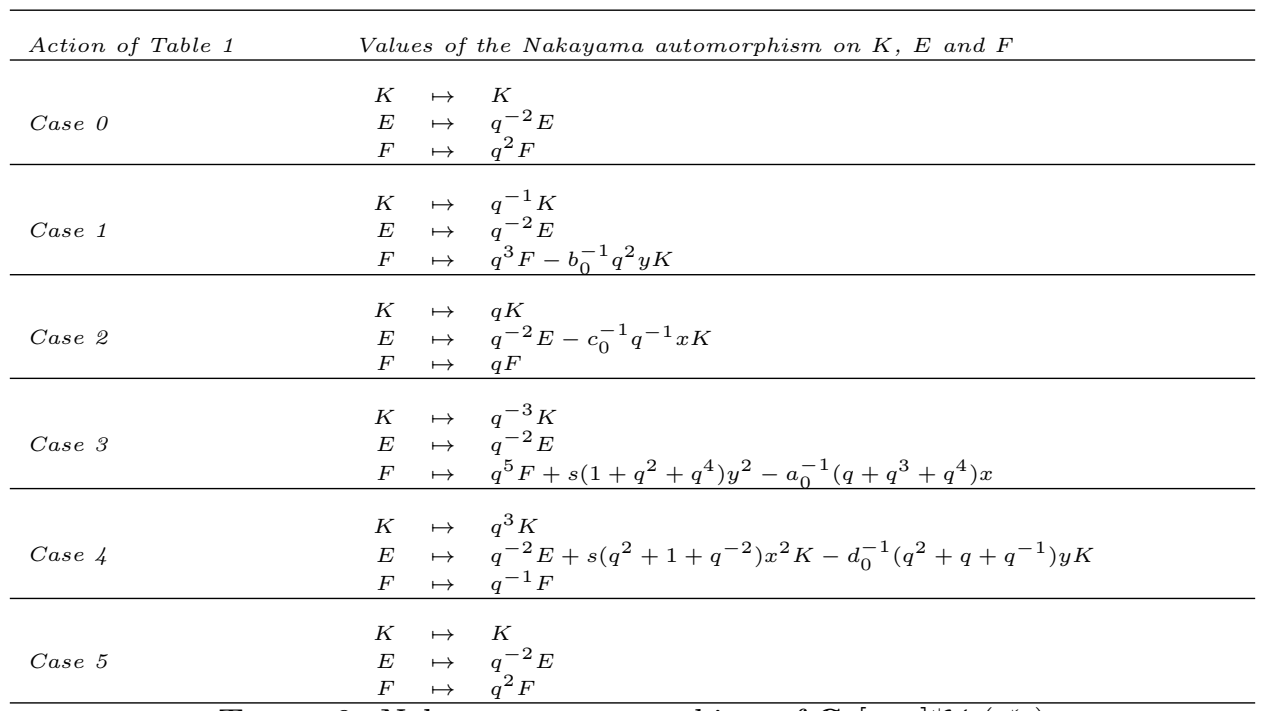

TABLE 6. Nakayama automorphism of $\mathbb{C}_{q}[x, y] \sharp \mathcal{U}_{q}\left(\mathfrak{s l}_{2}\right)$ 
Proof. It follows from 7.3.1 that $\mathbb{C}_{q}[x, y] \sharp \mathcal{U}_{q}\left(\mathfrak{s l}_{2}\right)$ is skew Calabi-Yau and admits $\mu_{A} \sharp\left(\theta_{\text {whdet }} \circ \mu_{H}\right)$ as a Nakayama automorphism, where

- $\mu_{A}$ is as in 8.1.0.4,

- whdet and $\theta_{\text {whdet }}$ are as in 8.4 .

- $\mu_{H}=S^{-2} \circ \Xi_{\int_{\ell}^{r}}^{r}$.

Note that $\Xi_{\int_{\ell}}^{r}=\operatorname{Id}_{H}$ because $\int_{\ell}=\epsilon$ (see 8.1.0.1), and $S^{-2}$ is given by $K \mapsto K$, $E \mapsto q^{-2} E$ and $F \mapsto q^{2} F$. The description given in Table 6 therefore follows from the one given in Table 5 .

Remark. (1) In case 5, the given Nakayama automorphism is the inner automorphism of $\mathbb{C}_{q}[x, y] \sharp \mathcal{U}_{q}\left(\mathfrak{s l}_{2}\right)$ associated with $K^{-1}$. Accordingly, the smashproduct is Calabi-Yau.

(2) This fact can be recovered from the theorem of 7.4.2. Indeed, in case 5, any action of $\mathcal{U}_{q}\left(\mathfrak{s l}_{2}\right)$ preserves the grading of $\mathbb{C}_{q}[x, y]$. Moreover the weak homological determinant is a homological determinant, which is moreover trivial (see Table 4). Therefore, taking $h_{0}=K^{-1}$ and $k_{A}=1$ in 7.4.2 yields the desired conclusion.

\section{Applichtions to Artin-Schelter algebras}

Assume that $A$ is an augmented $H$-module algebra and that the antipode of $H$ is invertible. This section investigates when $\Lambda$ is Artin-Schelter Gorenstein/regular. Sufficient conditions for this to be the case are presented in Section 9.3 using an analogue of Stefan's spectral sequence presented in Section 9.2 For this purpose, some useful module structures are introduced in Section 9.1 .

9.1. Useful module structures. In the rest of the text, whenever $M, N$ are left $H$-modules, the following structure of left $H$-module is considered on $\operatorname{Hom}_{\mathbb{k}}(M, N)$

$$
(h \rightarrow f)(m)=h_{2} \rightarrow f\left(S^{-1}\left(h_{1}\right) \rightarrow m\right) .
$$

Also $M \otimes N$ is considered as a left $H$-module for the following action

$$
h \rightarrow(m \otimes n)=h_{1} \rightarrow m \otimes h_{2} \rightarrow n .
$$

Assume that $M, N$ are left $\Lambda$-modules.

It is proved in [16, (1)] that $\operatorname{Hom}_{A}(M, N)$ is an $H$-submodule of $\operatorname{Hom}_{\mathbb{k}}(M, N)$. Moreover, according to [16, Lemma 2.2], the following canonical mapping is welldefined and bijective.

$$
\begin{aligned}
\operatorname{Hom}_{H}\left(H_{H} \mathbb{k}, \operatorname{Hom}_{A}(M, N)\right) & \cong \operatorname{Hom}_{\Lambda}(M, N) \\
\phi & \mapsto \phi(1) .
\end{aligned}
$$

In order to analyse the spectral sequence mentioned earlier, it is necessary to compare objects such as $\operatorname{Hom}_{A}(M, A) \otimes H$ and $\operatorname{Hom}_{A}(M, \Lambda)$.

Firstly, consider $\operatorname{Hom}_{A}(M, \Lambda)$. The natural structure of right $\Lambda$-module on $\operatorname{Hom}_{A}(M, \Lambda)$ commutes with the action of $H$. Hence, $\operatorname{Hom}_{A}(M, \Lambda)$ is an $H-\Lambda$ bimodule.

Secondly, consider $\operatorname{Hom}_{A}(M, A) \otimes H$. In view of 9.1.0.1 and 9.1.0.2, it is a left $H$-module. It is also a right $\Lambda$-module for the action defined by $(f \otimes \ell) a h=$ $f \cdot\left(\ell_{1} \rightarrow a\right) \otimes \ell_{2} h$ (here $\operatorname{Hom}_{A}(M, A)$ is naturally a right $A$-module). Given that the structures of left $H$-module and right $A$-module of $\operatorname{Hom}_{A}(M, A)$ are compatible in the following sense

$$
h \rightarrow(f a)=\left(h_{1} \rightarrow f\right)\left(h_{2} \rightarrow a\right),
$$

it follows that $\operatorname{Hom}_{A}(M, A) \otimes H$ is also an $H-\Lambda$-bimodule. 
For these structures of $H-\Lambda$-bimodule, the following canonical mapping is $H \otimes \Lambda^{\mathrm{op}}$-linear

$$
\begin{aligned}
\operatorname{Hom}_{A}(M, A) \otimes H & \rightarrow \operatorname{Hom}_{A}(M, \Lambda) \\
f \otimes \ell & \mapsto(m \mapsto f(m) \ell) .
\end{aligned}
$$

Note that it is a functorial isomorphism if $M$ is finitely presented in $\bmod (A)$ of if $\operatorname{dim}_{\mathbb{k}} H<\infty$.

Using projective resolutions to construct Ext-spaces, the previous considerations entail the following result.

Lemma. Let $M, N$ be left $\Lambda$-modules and let $p$ be a natural integer.

(1) There is a functorial structure of left $H$-module on $\operatorname{Ext}_{A}^{p}(M, N)$ which coincides with the one introduced previously when $p=0$.

(2) Taking into account the natural structure of right $\Lambda$-module, $\operatorname{Ext}_{A}^{p}(M, \Lambda)$ is then an $H-\Lambda$-bimodule.

(3) Taking into account the natural structure of right $\Lambda$-module such that

$$
(e \otimes \ell) a h=e\left(\ell_{1} \rightarrow a\right) \otimes \ell_{2} h,
$$

$\operatorname{Ext}_{A}^{p}(M, A) \otimes H$ is then an $H-\Lambda$-bimodule.

(4) There is a functorial morphism of $H-\Lambda$-bimodules $\operatorname{Ext}_{A}^{p}(M, A) \otimes H \rightarrow$ $\operatorname{Ext}_{A}^{p}(M, \Lambda)$. If $M$ has a resolution in $\bmod (A)$ by finitely generated projectives, then this is an isomorphism.

\subsection{A spectral sequence for the cohomology of augmented algebras.}

9.2.1. The following is an analogue of the spectral sequence constructed by Stefan ([32, Theorem 3.3]) for the Hochschild cohomology on Hopf-Galois extension. The proof is also analogous and omitted.

Proposition. Let $M, N$ be left $\Lambda$-modules. There is a Grothendieck spectral sequence functorial in $M$ and $N$

$$
\operatorname{Ext}_{H}^{p}\left({ }_{H} \mathbb{k}, \operatorname{Ext}_{A}^{q}(M, N)\right) \Rightarrow \operatorname{Ext}_{\Lambda}^{p+q}(M, N) \text {. }
$$

9.2.2. In view of determining when $\Lambda$ has the Artin-Schelter property, it is useful to simplify the $E_{2}$ term of the spectral sequence.

Lemma. Assume that ${ }_{H} \mathbb{k}$ has a resolution in $\bmod (H)$ by finitely generated projectives. Let $M \in \bmod (\Lambda)$ admit a resolution in $\bmod (A)$ by finitely generated projectives. Then, for every $p, q \in \mathbb{N}$, there is an isomorphism of right $\Lambda$-modules

$$
\operatorname{Ext}_{H}^{p}\left({ }_{H} \mathbb{k}, \operatorname{Ext}_{A}^{q}(M, \Lambda)\right) \simeq \operatorname{Ext}_{A}^{q}(M, A) \otimes \operatorname{Ext}_{H}^{p}\left(H_{H} \mathbb{k}, H\right)
$$

where the module structure of the right-hand side term is defined by

$$
\left(e_{M} \otimes e_{H}\right) a h=\left(S^{-1}\left(h_{1}\right) \rightarrow\left(e_{M} a\right)\right) \otimes e_{H} h_{2} .
$$

Proof. Let $P \rightarrow{ }_{H} \mathbb{k}$ be a resolution by finitely generated projective left $H$-modules. Deriving 9.1.0.4 yields an analogous identity in $\operatorname{Ext}_{A}^{q}(M, A)$. Consider the following action of $\Lambda$ on $\operatorname{Ext}_{A}^{q}(M, A) \otimes \operatorname{Hom}_{H}(P, H)$

$$
\left(e_{M} \otimes \varphi\right) a h:=\left(S^{-1}\left(h_{1}\right) \rightarrow\left(e_{M} a\right)\right) \otimes \varphi h_{2} .
$$

It is a structure of complex of right $\Lambda$-modules. Therefore, the action of $\Lambda$ on $\operatorname{Ext}_{A}^{q}(M, A) \otimes \operatorname{Ext}_{H}^{p}\left({ }_{H} \mathbb{k}, H\right)$ given in the statement of the lemma is a structure of right $\Lambda$-module.

The $H-\Lambda$-bimodules $\operatorname{Ext}_{A}^{q}(M, \Lambda)$ and $\operatorname{Ext}_{A}^{q}(M, A) \otimes H$ are isomorphic (see (9.1.0.5). Consider the morphism of complexes

$$
\begin{aligned}
\theta: \quad \operatorname{Ext}_{A}^{q}(M, A) \otimes \operatorname{Hom}_{H}(P, H) & \rightarrow \operatorname{Hom}_{H}\left(P, \operatorname{Ext}_{A}^{q}(M, A) \otimes H\right) \\
e_{M} \otimes \varphi & \mapsto\left(p \mapsto\left(\varphi(p)_{1} \rightarrow e_{M}\right) \otimes \varphi(p)_{2}\right) .
\end{aligned}
$$


Note that $\theta\left(e_{M} \otimes \varphi\right)$ is indeed $H$-linear because $\varphi$ is $H$-linear and because of the definition of the action of $H$ on $\operatorname{Ext}_{A}^{q}(M, A) \otimes H$ on the left (see 9.1.0.2). Since $\operatorname{Ext}_{A}^{q}(M, A) \otimes H \in \bmod \left(H \otimes \Lambda^{\mathrm{op}}\right)\left(\right.$ see 9.1), then $\operatorname{Hom}_{H}\left(P, \operatorname{Ext}_{A}^{q}(M, A) \otimes H\right) \in$ $\bmod \left(\Lambda^{\mathrm{op}}\right)$.

The mapping $\theta$ is $\Lambda$-linear. Indeed, let $e_{M} \otimes \varphi \in \operatorname{Ext}_{A}^{q}(M, A) \otimes \operatorname{Hom}_{H}(P, H)$, $a \in A, h \in H$ and $p \in P$. Then,

$$
\begin{aligned}
& \left(\theta\left(e_{M} \otimes \varphi\right) a h\right)(p) \quad=\quad \theta\left(e_{M} \otimes \varphi\right)(p) a h \\
& =\left(\varphi(p)_{1} \rightarrow e_{M} \otimes \varphi(p)_{2}\right) a h \\
& =\left(\varphi(p)_{1} \rightarrow e_{M}\right)\left(\varphi(p)_{2} \rightarrow a\right) \otimes \varphi(p)_{3} h \\
& \begin{array}{lll} 
& = & \varphi(p)_{1} \rightarrow\left(e_{M} a\right) \otimes \varphi(p)_{2} h \\
\theta\left(\left(e_{M} \otimes \varphi\right) a h\right)(p) & \stackrel{9.1 .0 .4}{=} & \theta\left(S^{-1}\left(h_{1}\right) \rightarrow\left(e_{M} a\right) \otimes \varphi h_{2}\right)(p)
\end{array} \\
& =\left(\varphi h_{2}\right)(p)_{1} \rightarrow\left(S^{-1}\left(h_{1}\right) \rightarrow\left(e_{M} a\right)\right) \otimes\left(\varphi h_{2}\right)(p)_{2} \\
& =\left(\varphi(p)_{1} h_{2}\right) \rightarrow\left(S^{-1}\left(h_{1}\right) \rightarrow\left(e_{M} a\right)\right) \otimes \varphi(p)_{2} h_{3} \\
& =\varphi(p)_{1} \rightarrow\left(e_{M} a\right) \otimes \varphi(p)_{2} h,
\end{aligned}
$$

which explains why $\theta$ is $\Lambda$-linear.

In order to prove the lemma, it is therefore sufficient to prove that $\theta$ is bijective. When $P$ is replaced by $H$ in the description of $\theta$, the resulting mapping reduces to

$$
\begin{aligned}
\operatorname{Ext}_{A}^{q}(M, A) \otimes H & \rightarrow \operatorname{Ext}_{A}^{q}(M, A) \otimes H \\
e_{M} \otimes \varphi & \mapsto \varphi_{1} \rightarrow e_{M} \otimes \varphi_{2} .
\end{aligned}
$$

This is indeed bijective with inverse mapping given by $e_{M} \otimes \varphi \mapsto S^{-1}\left(\varphi_{1}\right) \rightarrow$ $e_{M} \otimes \varphi_{2}$. Now, since $P$ consists of finitely generated projective left $H$-modules, the previous considerations show that $\theta$ is bijective.

\subsection{The Artin-Schelter property of $A \sharp H$.}

9.3.1. Here is how the left Artin-Schelter property behaves under taking smash products. The dual statement for the right Artin-Schelter property holds true.

Proposition. Let $H$ be a Hopf algebra with invertible antipode. Assume that ${ }_{H} \mathbb{k}$ has a resolution in $\bmod (H)$ by finitely generated projectives. Let $A$ be an augmented $H$-module algebra. Assume that ${ }_{A} \mathbb{k}$ has a resolution in $\bmod (A)$ by finitely generated projectives. If $A$ and $H$ have the left Artin-Schelter property in dimension $n$ and $d$, respectively, then $A \sharp H$ has the left Artin-Schelter property in dimension $n+d$.

Proof. By assumption, $\operatorname{Ext}_{A}^{q}(\mathbb{k}, A)$ is one dimensional if $q=n$ and 0 otherwise. And $\operatorname{Ext}_{H}^{p}(H \mathbb{k}, H)$ is one dimensional if $p=d$ and 0 otherwise. Let $M={ }_{\Lambda} \mathbb{k}$ and $N=\Lambda$. The proposition therefore follows from 9.2.1 and 9.2 .2 .

9.3.2. Whenever $B$ is an augmented $\mathbb{k}$-algebra with left Artin-Schelter property in dimension $t$, there exists a unique algebra homomorphism $\lambda_{B}: B \rightarrow \mathbb{k}$ such that the right $B$-module structure of $\operatorname{Ext}_{B}^{t}(\mathbb{k}, B)$ is given by $e_{B} b=\lambda_{B}(b) e_{B}$ for every $e_{B} \in \operatorname{Ext}_{B}^{t}(\mathbb{k}, B)$.

In the setting of 9.3.1, the algebra homomorphism $\lambda_{A \sharp H}$ may be described in terms of $\lambda_{A}$ and $\lambda_{H}$. Since $\operatorname{Ext}_{A}^{n}\left(\mathbb{k}_{k}, A\right)$ is one dimensional, there exists an algebra homomorphism $\delta: H \rightarrow \mathbb{k}$ such that the left $H$-module structure of $\operatorname{Ext}_{A}^{n}(\mathbb{k}, A)$ is given by $h \rightarrow e_{A}=\delta(h) e_{A}$ for every $e_{A} \in \operatorname{Ext}_{A}^{n}(\mathbb{k}, A)$. Note that, when $H$ is finite dimensional and $A$ is connected graded and Artin-Schelter Gorenstein, then $\delta \circ S$ $\left(=\delta \circ S^{-1}\right)$ is the homological determinant defined in [23, Definition 3.3]. According to 9.2 .2 , the algebra homomorphism $\lambda_{A \sharp H}$ is given by

$$
\lambda_{A \sharp H}(a h)=\lambda_{A}(a) \delta\left(S^{-1}\left(h_{1}\right)\right) \lambda_{H}\left(h_{2}\right) .
$$


9.3.3. Here are sufficient conditions for $\Lambda$ to be Artin-Schelter regular.

Proposition. Let $H$ be a Hopf algebra with invertible antipode. Assume that ${ }_{H} \mathbb{k}$ has a resolution in $\bmod (H)$ by finitely generated projectives. Let $A$ be an augmented $H$-module noetherian algebra. Assume that $H$ and $A$ are Artin-Schelter regular in dimension $n$ and $d$, respectively. Then, $A \sharp H$ is Artin-Schelter regular in dimension $n+d$.

Proof. Since the antipode is invertible, then $\mathbb{k}_{H}$ has a resolution in $\bmod \left(H^{\mathrm{op}}\right)$ by finitely generated projectives. According to 9.3.1 and its dual version, it suffices to prove that gl.dim. $\Lambda \leqslant n+d$. This follows from 9.2.1. from its dual version and from the hypotheses on the global dimensions of $A$ and $H$.

9.3.4. In order to present an analog of the previous result for Artin-Schelter Gorenstein algebras it is necessary to deal with the finiteness of injective dimensions. This is taken care of by the following result.

Lemma. Let $H$ be a Hopf algebra with invertible antipode. Let $A$ be an $H$-module algebra. Let $\Lambda=A \sharp H$. Assume that $A$ is left noetherian. Then, $\operatorname{id}\left({ }_{\Lambda} \Lambda\right)<\infty$ under any of the two following conditions

(a) $H$ is finite-dimensional and $\operatorname{id}\left({ }_{A} A\right)<\infty$,

(b) $\operatorname{pd}_{H}\left(H_{H} \mathbb{k}\right)<\infty$ and $\operatorname{id}_{A}(A)<\infty$.

Proof. First, assume (a). Note that $\operatorname{id}\left({ }_{H} H\right)=0$, since all finite dimensional Hopf algebras are Frobenius, and that ${ }_{H} \mathbb{k}$ has a resolution in $\bmod (H)$ by finitely generated projectives. Any finitely generated left $\Lambda$-module is finitely generated as an $A$-module. Therefore, 9.2.1 and 9.2 .2 apply to any $M \in \bmod (\Lambda)$ which is finitely generated. For any such $M$, it follows that $\operatorname{Ext}_{\Lambda}^{m}(M, \Lambda)=0$ for $m>\operatorname{id}\left({ }_{A} A\right)$. Taking direct limits entails that $\operatorname{id}_{\Lambda}(\Lambda)<\infty$.

Next, assume (b). The following properties imply that $\operatorname{id}_{A}(\Lambda)<\infty$ :

- since $A$ is left noetherian any direct sum of injective left $A$-modules is an injective $A$-module (see [24, Proposition 3.46, p. 80]),

- $\operatorname{id}\left({ }_{A} A\right)<\infty$,

- $\Lambda \simeq A \otimes H$ as left $A$-modules.

The conclusion of the lemma then follows from 9.2.1 applied to $N=\Lambda$.

9.3.5. Here are sufficient conditions for $\Lambda$ to be Artin-Schelter Gorenstein.

Proposition. Let $H$ be a Hopf algebra. Let $A$ be an augmented $H$-module noetherian algebra which is moreover Artin-Schelter Gorenstein in dimension $n$.

(1) If $H$ is finite dimensional, then $\Lambda$ is Artin-Schelter Gorenstein in dimension $n$.

(2) If $H$ has Van den Bergh duality in dimension d, then $\Lambda$ is Artin-Schelter Gorenstein in dimension $n+d$.

Proof. Any finite dimensional Hopf algebra has an invertible antipode and is selfinjective. In particular, it is Artin-Schelter Gorenstein. Also, recall that, if $H$ has Van den Bergh duality, then its antipode is invertible, ${ }_{H} \mathbb{k}_{\mathrm{k}} \in \operatorname{per}(H)$ and $\mathbb{k}_{H} \in \operatorname{per}\left(H^{\mathrm{op}}\right)$, and $H$ has the Artin-Schelter property (see 3.5.1 and its dual version). Therefore (1) and (2) follow from 9.3.1, from 9.3.4 and their dual versions.

\section{ACKNOWLEDGEMENTS}

I thank an anonymous referee for several comments which improved the presentation of this article and for having pointed out an error in a previous version of the lemma in 7.4.1. 
Index of notation

\begin{tabular}{|c|c|c|}
\hline Symbol & Meaning & Location \\
\hline $\mathbb{k}$ & base field & Introduction \\
\hline$A$ & dg $H$-module $\mathbb{k}$-algebra & Introduction \\
\hline$A^{e}$ & $A \otimes_{\mathbb{k}} A^{\mathrm{op}}$ & Introduction \\
\hline$\mu_{A}$ & a Nakayama automorphism of $A$ & Introduction \\
\hline$H$ & Hopf $\mathbb{k}$-algebra & Introduction \\
\hline$S$ & antipode of $H$ & Introduction \\
\hline$\Lambda$ & smash product $A \sharp H$ & Introduction \\
\hline${ }^{\tau} M^{\sigma}$ & twisting of a bimodule by algebra automorphisms $\tau, \sigma$ & Introduction \\
\hline$\otimes$ & $\otimes_{\mathrm{k}}$ & 2.1 \\
\hline $\mathcal{C}(A)$ & category of left $\operatorname{dg} A$-modules & 2.1 \\
\hline $\mathcal{D}(A)$ & derived category of left $\operatorname{dg} A$-modules & 2.1 \\
\hline $\int_{\ell}, \int_{r}$ & left and right homological integrals & 3.1 \\
\hline$\Xi_{\bullet}^{\ell}, \Xi_{\bullet}^{r}$ & winding automorphisms & 3.1 \\
\hline - $\uparrow^{H^{e}}$ & functor from left $H$-modules to $H$-bimodules & 3.2 \\
\hline$\Delta_{i}$ & dg algebra whose left dg modules are $H_{S} 2 i$-equivariant dg $A$-bimodules & \begin{tabular}{|l|l|l}
4.1 \\
\end{tabular} \\
\hline$D \sharp^{\sigma} H$ & $\Lambda$-bimodule extension of an $H_{S^{2 i}}$-equivariant dg $A$-bimodule & 4.2 \\
\hline$H^{\sigma} \sharp D$ & & 4.2 .3 \\
\hline$\Pi_{n}(A)$ & Calabi-Yau completion of $A$ & Section 6 \\
\hline$\Pi_{n}(A, \alpha)$ & a deformed Calabi-Yau completion of $A$ & Section 6 \\
\hline$e_{A}$ & a free generator of the left $A$-module $\operatorname{Ext}_{A^{e}}^{n}\left(A, A^{e}\right)$ & 7.2 \\
\hline$\varphi_{A}$ & a cocycle representing $e_{A}$ & 7.2 \\
\hline whdet & weak homological determinant $H \rightarrow A$ & $\overrightarrow{7.2 .2}$ \\
\hline$\theta_{\text {whdet }}$ & algebra homomorphism $H \rightarrow \Lambda$ corresponding to whdet & 7.2 .2 \\
\hline hdet & homological determinant & 7.2 .4 \\
\hline$\mu_{\Lambda}, \mu_{H}$ & Nakayama automorphisms of $\Lambda$ and $H$ & 7.3 \\
\hline $\mathbb{C}_{q}[x, y]$ & the quantum plane & 8.1 \\
\hline $\mathcal{U}_{q}\left(\mathfrak{s l}_{2}\right)$ & the quantum enveloping algebra & 8.1 \\
\hline
\end{tabular}

\section{REFERENCES}

[1] C. Amiot. Cluster categories for algebras of global dimension 2 and quivers with potential. Ann. Inst. Fourier (Grenoble), 59(6):2525-2590, 2009.

[2] C. Amiot, O. Iyama, and I. Reiten. Stable categories of Cohen-Macaulay modules and cluster categories. Amer. J. Math., 137(3):813-857, 2015.

[3] M. Artin and W. F. Schelter. Graded algebras of global dimension 3. Adv. in Math., 66(2):171216, 1987.

[4] R. Berger and A. Pichereau. Calabi-Yau algebras viewed as deformations of Poisson algebras. Algebr. Represent. Theory, 17(3):735-773, 2014.

[5] K. A. Brown and K. R. Goodearl. Homological aspects of Noetherian PI Hopf algebras of irreducible modules and maximal dimension. J. Algebra, 198(1):240-265, 1997.

[6] K. A. Brown and J. J. Zhang. Dualising complexes and twisted Hochschild (co)homology for Noetherian Hopf algebras. J. Algebra, 320(5):1814-1850, 2008.

[7] S. Chemla. Rigid dualizing complex for quantum enveloping algebras and algebras of generalized differential operators. J. Algebra, 276(1):80-102, 2004.

[8] A. Chirvasitu, R. Kanda, and S. P. Smith. New Artin-Schelter regular and Calabi-Yau algebras via normal extensions. ArXiv e-prints, June 2017.

[9] L. de Thanhoffer de Völcsey and M. Van den Bergh. Explicit models for some stable categories of maximal Cohen-Macaulay modules. Math. Res. Lett., 23(5):1507-1526, 2016.

[10] S. Duplij and S. Sinel'shchikov. Classification of $U_{q}\left(\mathfrak{s l}_{2}\right)$-module algebra structures on the quantum plane. Zh. Mat. Fiz. Anal. Geom., 6(4):406-430, 436, 439, 2010.

[11] P. Etingof and V. Ginzburg. Noncommutative del Pezzo surfaces and Calabi-Yau algebras. J. Eur. Math. Soc. (JEMS), 12(6):1371-1416, 2010. 
[12] M. Farinati. Hochschild duality, localization, and smash products. J. Algebra, 284(1):415-434, 2005.

[13] V. Ginzburg. Calabi-Yau algebras. arXiv:math/0612139 v3, 2006.

[14] J. Goodman and U. Krähmer. Untwisting a twisted Calabi-Yau algebra. J. Algebra, 406:272289,2014

[15] J.-W. He, F. Van Oystaeyen, and Y. Zhang. Cocommutative Calabi-Yau Hopf algebras and deformations. J. Algebra, 324(8):1921-1939, 2010.

[16] J.-W. He, F. Van Oystaeyen, and Y. Zhang. Hopf algebra actions on differential graded algebras and applications. Bull. Belg. Math. Soc. Simon Stevin, 18(1):99-111, 2011.

[17] F. Ischebeck. Eine Dualität zwischen den Funktoren Ext und Tor. J. Algebra, 11:510-531, 1969.

[18] P. Jørgensen and J. J. Zhang. Gourmet's guide to Gorensteinness. Adv. Math., 151(2):313$345,2000$.

[19] A. Kaygun. Hopf-Hochschild (co)homology of module algebras. Homology, Homotopy Appl., $9(2): 451-472,2007$.

[20] B. Keller. Deriving DG categories. Ann. Sci. École Norm. Sup. (4), 27(1):63-102, 1994

[21] B. Keller. Deformed Calabi-Yau completions. J. Reine Angew. Math., 654:125-180, 2011. With an appendix by Michel Van den Bergh.

[22] B. Keller and D. Yang. Derived equivalences from mutations of quivers with potential. Adv. Math., 226(3):2118-2168, 2011.

[23] E. Kirkman, J. Kuzmanovich, and J. J. Zhang. Gorenstein subrings of invariants under Hopf algebra actions. J. Algebra, 322(10):3640-3669, 2009.

[24] T. Y. Lam. Lectures on modules and rings, volume 189 of Graduate Texts in Mathematics. Springer-Verlag, New York, 1999

[25] P. Le Meur. Crossed-products of Calabi-Yau algebras by finite groups. arXiv:1006.1082 [math.RA], 2010.

[26] L.-Y. Liu, Q.-S. Wu, and C. Zhu. Hopf action on Calabi-Yau algebras. In New trends in noncommutative algebra, volume 562 of Contemp. Math., pages 189-209. Amer. Math. Soc., Providence, RI, 2012.

[27] I. Mori and S. P. Smith. m-Koszul Artin-Schelter regular algebras. J. Algebra, 446:373-399, 2016.

[28] S. Mozgovoy and M. Reineke. On the noncommutative Donaldson-Thomas invariants arising from brane tilings. Adv. Math., 223(5):1521-1544, 2010.

[29] C. Negron. Spectral sequences for the cohomology rings of a smash product. J. Algebra, 433:73-106, 2015

[30] M. Reyes, D. Rogalski, and J. J. Zhang. Skew Calabi-Yau algebras and homological identities. Adv. Math., 264:308-354, 2014.

[31] M. Reyes, D. Rogalski, and J. J. Zhang. Skew Calabi-Yau triangulated categories and Frobenius Ext-algebras. Trans. Amer. Math. Soc., 369(1):309-340, 2017.

[32] D. Ştefan. Hochschild cohomology on Hopf Galois extensions. J. Pure Appl. Algebra, 103(2):221-233, 1995.

[33] M. Van den Bergh. Non-commutative homology of some three-dimensional quantum spaces. K-Theory, 8:213-220, 1994.

[34] M. van den Bergh. A relationr between Hochschild homology and cohomology for Gorenstein rings. Proc. Amer. Math. Soc., 126(5):1345-1348, 1998.

[35] M. Wambst. Complexes de Koszul quantiques. Ann. Inst. Fourier (Grenoble), 43(4):10891156, 1993.

[36] D. Wang and Y. Ke. The Calabi-Yau property of twisted smash products. J. Algebra Appl., 13(3):1350118, 19, 2014

[37] Q.-S. Wu and C. Zhu. Skew group algebras of Calabi-Yau algebras. J. Algebra, 340:53-76, 2011.

[38] A. Yekutieli. The rigid dualizing complex of a universal enveloping algebra. J. Pure Appl. Algebra, 150(1):85-93, 2000.

[39] W.-K. Yeung. Relative Calabi-Yau completions. arXiv:1612.06352 [math.RT], 2016.

[40] X. Yu. Hopf-Galois objects of Calabi-Yau Hopf algebras. J. Algebra Appl., 15(10):1650194, $19,2016$.

(Patrick Le Meur) Laboratoire de Mathématiques Blaise Pascal, UMR6620 CNRS, Université Clermont Auvergne, Campus des Cézeaux, 3 place Vasarely, 63178 Aubière CEDEX, FRANCE

Current address: Université de Paris, Sorbonne Université, CNRS, Institut de Mathématiques de Jussieu-Paris Rive Gauche, IMJ-PRG, F-75013, Paris, France

Email address: patrick.le-meur@imj-prg.fr 